\title{
A Laboratory Study of Localized Boundary Mixing in a Rotating Stratified Fluid
}

by

\author{
Judith R. Wells \\ B.S. University of Massachusetts, Boston 1996 \\ M.C.P. University of California, Berkeley 1968 \\ B.A. Harvard University, 1966 \\ Submitted in partial fulfillment of the \\ requirements for the degree of \\ Doctor of Philosphy \\ at the \\ MASSACHUSETTS INSTITUTE OF TECHNOLOGY \\ and the \\ WOODS HOLE OCEANOGRAPHIC INSTITUTION
}

February 2003

() 2003 Woods Hole Oceanographic Institution

All rights reserved.

Signature of Author.

Joint Program in Physical Oceanography

Massachusetts Institute of Technology

Woods Hole Oceanographic Institution

September 12, 2002

Certified by.............

Karl R. Helfrich

Senior Scientist

Woods Hole Oceanographic Institution

Accepted by.

Carl Wunsch

Chairman, Joint Committee for Physical Oceanography Massachusetts Institute of Technology

Woods Hole Oceanographic Institution 



\title{
A Laboratory Study of Localized Boundary Mixing in a Rotating Stratified Fluid
}

\author{
by \\ Judith R. Wells \\ Submitted in partial fulfillment of the requirements for the degree of \\ Doctor of Philosophy at the Massachusetts Institution of Technology \\ and the Woods Hole Oceanographic Institution
}

September 12, 2002

\begin{abstract}
Oceanic observations indicate that abyssal mixing is localized in regions of rough topography. How locally mixed fluid interacts with the ambient fluid is an open question. Laboratory experiments explore the interaction of mechanically induced boundary mixing and an interior body of linearly stratified rotating fluid. Turbulence is generated by a vertically oscillating horizontal bar, located at middepth along the tank wall. The turbulence forms a region of mixed fluid which quickly reaches a steady state height and collapses into the interior. The mixed layer thickness, $h_{m} \propto \gamma\left(\frac{\omega}{N}\right)^{1 / 2}$, is independent of the Coriolis frequency $f . N$ is the buoyancy frequency, $\omega$ is the bar frequency, and the constant, $\gamma=1 \mathrm{~cm}$, is empirically determined by bar mechanics.

In initial experiments, the bar is exposed on three sides. Mixed fluid intrudes directly into the interior as a radial front of uniform height, rather than as a boundary current. Mixed fluid volume grows linearly with time, $V \propto\left(\frac{N}{f}\right)^{3 / 2} h_{m}^{3}(f t)$. The circulation patterns suggest a model of unmixed fluid being laterally entrained with velocity, $u_{e} \sim N h_{m}$, into the sides of a turbulent zone with height $h_{m}$ and width $L_{f} \equiv \gamma\left(\frac{\omega}{f}\right)^{1 / 2}$, where $L_{f}$ is an equilibrium scale associated with rotational control of bar-generated turbulence. In accord with the model, outflux is constant, independent of stratification and restricted by rotation, $Q \propto h_{m} L_{f} u_{e}$.
\end{abstract}

Later experiments investigate the role of lateral entrainment by confining the sides of the mixing bar between two walls, forming a channel open to the basin at one end. A small percentage of exported fluid enters a boundary current, but the bulk forms a cyclonic circulation in front of the bar. As the recirculation region expands to fill the channel, it restricts horizontal entrainment into the turbulent zone. The flux of mixed fluid decays with time. $Q \propto L_{C} h_{m}\left(L_{f} u_{e}\right)^{1 / 2} t^{-1 / 2}$, where $L_{C}$ is the channel width. The production of mixed fluid depends on the size of the mixing zone as well as on the balance between turbulence, rotation and stratification. As horizontal entrainment is shut down, longterm production of mixed fluid may be determined through much weaker vertical entrainment. Ultimately, the export of mixed fluid from the channel is restricted to the weak boundary current.

Thesis Supervisor: Karl R. Helfrich

Title: Senior Scientist, Woods Hole Oceanographic Institution 


\section{Acknowledgments}

I would like to take this opportunity to thank my advisor, Karl Helfrich, for giving me both the freedom to define my research and the guidance to stay on course. His continuing interest, careful criticism and perceptive questions have added greatly to my graduate education. I would also like to thank my thesis committee members, John Marshall, Ray Schmitt, Jack Whitehead and Carl Wunsch, for their availability, advice and suggestions, and Sonya Legg for kindly agreeing to chair my defense. Special thanks are due to John and Jack and to Richard Wardle for providing me with an introduction to oceanographic research and inspiring me to apply to graduate school. Claudia Cenedese, Joe Pedlosky and Kurt Polzin provided guidance and encouragement along the way, and Keith Bradley and John Salzig made this thesis based on laboratory work realizable.

The students and staff at WHOI and MIT have made my life as a graduate student a pleasure. I give my special thanks to my close classmates and quasiclassmates, Juan Botella, Albert Fischer, Pål Erik Isachsen, Steve Jayne, Galen McKinley, Avon Russell, Caixia Wang and Sandra Werner, to my housemates Domenique Decou Gummow and Mirta Teichberg, and to my Clarkmates, Jen Georgen, Deb Glickson, Heather Hunt Furey and all the PO postdocs.

Thanks, everyone!

This work was supported by the Ocean Ventures Fund, the Westcott Fund and the WHOI Education Office. Financial support was also provided by the National Science Foundation through grant OCE-9616949. 


\section{Contents}

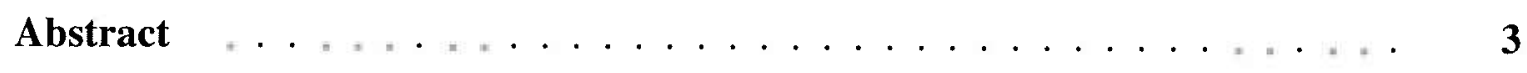

Acknowledgments $\ldots \ldots \ldots \ldots \ldots \ldots \ldots \ldots \ldots \ldots \ldots$

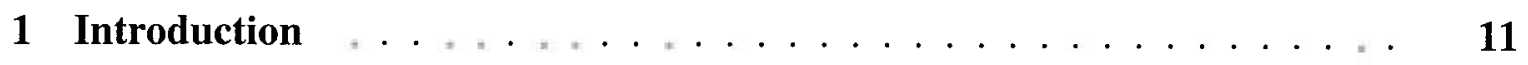

2 A Review of Laboratory Experiments with Mechanically-Induced

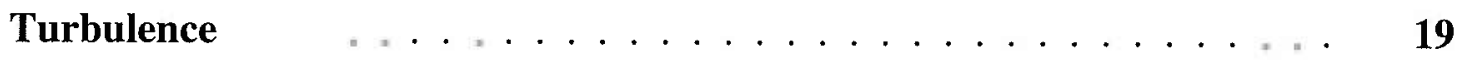

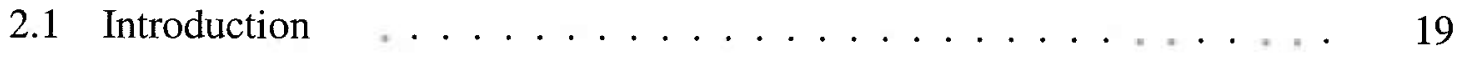

2.2 Properties of grid-generated turbulence $\ldots \ldots \ldots \ldots . \ldots 21$

2.3 Turbulence and stratification $\ldots \ldots \ldots \ldots \ldots . \ldots \ldots$

2.3.a Buoyant inhibition of turbulence $\ldots \ldots \ldots \ldots . \ldots 23$

2.3.b Mixed layer deepening and the entrainment hypothesis _... . 25

2.3.c Localized mixing and mixed layer deepening . . . . . . 27

2.3.d Vertical boundary mixing . . . . . . . . . . . . . 29

2.4 Turbulence and rotation $\ldots \ldots \ldots \ldots \ldots \ldots$

2.4.a Rotational inhibition of turbulence . . . . . . . . . 33

2.4.b Rotational effects on vertical entrainment . . . . . . . 36

2.5 Rotating, stratified experiments . . . . . . . . . . . . . 37

2.6 Conclusion . . . . . . . . . . . . . . . . . . . 41

3 The Laboratory Setup . . . . . . . . . . . . . . . . . . . 43

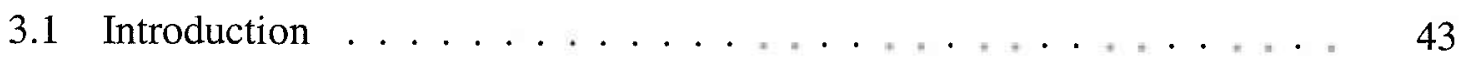

3.2 Experimental apparatus $\ldots \ldots \ldots \ldots . \ldots \ldots 4 . \ldots \ldots$

3.3 Properties of bar-generated turbulence $\ldots \ldots \ldots \ldots . \ldots 47$ 
3.4 Measurement methods . . . . . . . . . . . . . . . . . . 56

3.5 Experimental parameters . . . . . . . . . . . . . 57

4 Localized Mixing at a Vertical Boundary $\ldots \ldots \ldots 61$

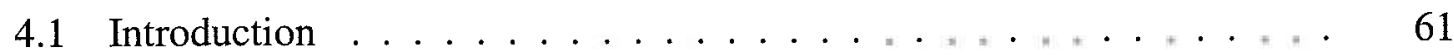

4.2 Qualitative description . . . . . . . . . . . . 62

4.2.a Export of mixed fluid from the turbulent zone . . . . . . 62

4.2.b Entrainment of unmixed fluid into the turbulent zone \# . . . 67

4.3 Quantitative analysis and scaling . . . . . . . . . . . . 69

4.3.a Data sources ... . . . . . . . . . . . . . 70

4.3.b Vertical mixing and layer height $\ldots \ldots \ldots \ldots \ldots$

4.3.c Advance of the mixed layer front . . . . . . . . . . . 74

4.3.d Lateral spreading of the mixed layer . . . . . . . . . . 77

4.3.e Volume of mixed fluid . . . . . . . . . . . 80

4.4 A model for mixing in the presence of rotation . . . . . . . . 81

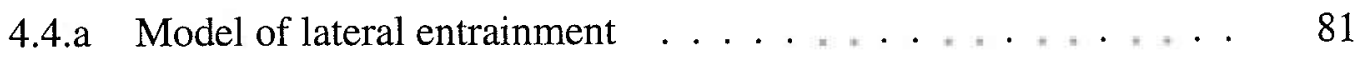

4.4.b Model of vertical entrainment f . . . . . . . . . 82

4.4.c Variations in bar length . . . . . . . . . . 85

4.5 The limits of rotational effects $\ldots \ldots \ldots \ldots \ldots$

4.6 Discussion . . . . . . . . . . . . . . . . . . . . . . 91

5 Mixing in a Channel $\ldots \ldots \ldots \ldots \ldots$

5.1 Introduction $\ldots \ldots \ldots \ldots \ldots \ldots \ldots \ldots \ldots \ldots \ldots \ldots \ldots$

5.2 Qualitative description $\ldots \ldots \ldots \ldots \ldots \ldots \ldots \ldots \ldots$

5.2.a Evolution of the mixed layer $\ldots \ldots \ldots \ldots \ldots$

5.2.b Variations in flow patterns . . . . . . . . . . . 102

5.2.c A comparison with previous work . . . . . . . . 104

5.3 Quantitative analysis and scaling . . . . . . . . . . . 105

5.3.a Height of the mixed layer . . . . . . . . . . . 105 
5.3.b Recirculation region ................. . . 107

5.3.c Boundary current ......................... 113

5.3.d Variation in channel width ............... 114

5.4 Geostrophic transport calculations . . . . . . . . . . . . . 119

5.5 Mixing in the absence of rotation: $f=0 \ldots \ldots \ldots . \ldots . \ldots . \ldots 124$

5.6 Summary . . . . . . . . . . . . . . . . . . . . . . . 129

6 Discussion . . . . . . . . . . . . . . . . . . . . . 133

6.1 Localized mixing in the laboratory . . . . . . . . . . . . 133

6.2 Localized mixing in the ocean . . . . . . . . . . . . . 136

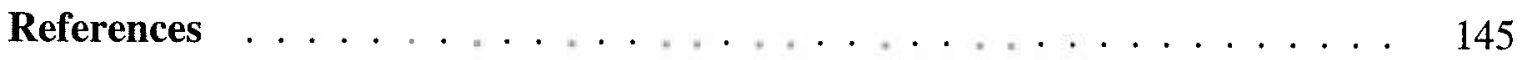




\section{Chapter 1}

\section{Introduction}

Turbulent mixing in the deep ocean is a fundamental component of the meridional overturning circulation. Bottom waters originate through surface heat loss in high latitude regions such as the Labrador Sea in the North Atlantic and the Weddell Sea off Antarctica. The cold dense water formed in these marginal seas sinks, spreads equatorward and upwells in the ocean basins. As it rises it is modified by heat mixed downward from sun-warmed surface layers.

The mixing required to restore surface properties to abyssal waters was first inferred from basin scale property distributions. Munk (1966) found the temperature and salinity distributions in the central Pacific well described by a simple advective-diffusive density balance,

$$
w \frac{\partial \rho}{\partial z}=\kappa \frac{\partial^{2} \rho}{\partial z^{2}},
$$

where $\rho$ is density, $z$ is the vertical or cross isopycnal coordinate, $w$ is vertical velocity, and $\kappa$ is the turbulent diffusivity coefficient. A basinwide upwelling velocity, estimated from the influx of Antarctic bottom water as $w \approx 10^{-2} \mathrm{~m} / \mathrm{s}$, implied a diapycnal diffusivity, $K \approx 10^{-4} \mathrm{~m}^{2} / \mathrm{s}$.

Subsequent efforts to define diapycnal fluxes showed considerable variability in mixing rates between the upper ocean and the abyss, and between mislatitude gyres and the equatorial regions. Early microstructure surveys found thermocline mixing rates an 
order of magnitude weaker than the basinwide value inferred by Munk (Gregg, 1980; Gregg and Sanford, 1980). Inverse calculations, constrained by large hydrographic data sets, also estimated upper thermocline diffusivites of $K \approx 10^{-5} \mathrm{~m}^{2} / \mathrm{s}$ for the midocean gyres (Gregg, 1987). In the abyss, measurements of flow through deep passages were used to formulate heat and mass budgets and evaluate mixing rates. Whitehead and Worthington (1982) calculated heat and salt fluxes in the North Atlantic due to northward flow of Antarctic Bottom Water through a passage at $4^{\circ} \mathrm{N}$. Hogg et al. (1982) inferred diapycnal diffusivities from a mass and heat budget for the bottommost layers of flow through the Brazil Basin. Volume changes in the chosen layers were explained in terms of a cross-isothermal diffusive flux and an opposing advective flux. The Hogg et al. analysis established a vertical diffusion coefficient, $\kappa \approx 3 \times 10^{-4} \mathrm{~m}^{2} / \mathrm{s}$. The Brazil Basin budget, like Munk's model for the Pacific, accounted for known sources of bottom water with a uniform spatially-averaged pelagic mixing rate of $O\left(10^{-4}\right) \mathrm{m}^{2} / \mathrm{s}$.

Subsequent measurements of velocity and temperature microstructure and observations of turbulently enhanced tracer diffusion have made it possible to evaluate the abyssal mixing rates on a more localized basis. Spatial variations in deep ocean mixing have been identified by microstructure surveys in the eastern North Pacific and North Atlantic (Lueck and Mudge, 1997; Toole et al., 1994, 1997). Similar variablity is indicated in the western South Atlantic by the Brazil Basin Tracer Release Experiment (BBTRE), a combined microstructure and tracer study (Polzin et al., 1997; Ledwell et al., 2000). In the interior away from boundaries, the measurements show diffusivities of $\kappa \approx 10^{-5} \mathrm{~m}^{2} / \mathrm{s}$, an order of magnitude below the uniform $10^{-4} \mathrm{~m}^{2} / \mathrm{s}$ inferred by Munk and Hogg et al. However, over the flanks of Pacific seamounts and above the slopes and fracture zones of the MidAtlantic Ridge, mixing rates exceed $10^{-4} \mathrm{~m}^{2} / \mathrm{s}$ and climb as high as $10^{-3} \mathrm{~m}^{2} / \mathrm{s}$. In general, weak mixing is associated with smooth topography and intensified mixing with rough topography.

In an examination of water properties in the Brazil Basin, Morris et al. (2001) updated the Hogg et al. (1982) heat and mass budget with comprehensive data from the World 
Ocean Circulation Experiment (WOCE), and used the BBTRE microstructure data to provide an independent measure of mixing rates. They categorize the bathymetry as smooth or rough. Smooth regions are assigned a diffusivity of $10^{-5} \mathrm{~m}^{2} / \mathrm{s}$. Rough regions are assigned profiles of turbulent kinetic energy specified as functions of height above bottom with separate profiles for crests, canyons and slopes based on the BBTRE data. The computed diffusivities, when spatially averaged over the basin, give a mean value, $\bar{K}=1-3\left(\times 10^{-4} \mathrm{~m}^{2} / \mathrm{s}\right)$, consistent with the values inferred from the 1982 and 2001 budget analyses. The localized regions of enhanced diffusivity provide sufficient mixing to account for the heat and mass balances. The basinwide averaging, however, does not address the mechanics of distributing mixed properties within the basin.

Munk and Wunsch (1998) suggest that locally generated salinity and temperature characteristics are exported from mixing sites along neutral surfaces. They interpret the diffusivity coefficient $\mathrm{K}$, a constant in the one-dimensional Munk model (1.1), as a surrogate for a number of small concentrated sources of buoyancy flux, and extend the advective-diffusive balance to include a horizontal advection term,

$$
\kappa \frac{\partial^{2} \rho}{\partial z^{2}}=w \frac{\partial \rho}{\partial z}+u \frac{\partial \rho}{\partial x},
$$

where $x$ is the horizontal or along isopycnal coordinate, and $u$ is the horizontal velocity. Locally mixed fluid in a stratified environment creates horizontal density differences and the pressure gradient drives an outflux of mixed fluid. Geostrophic adjustment in the presence of rotation may generate rim currents along the mixed fluid front or form boundary currents. Baroclinic instability along the edges of these currents mixes fluid still further into the stratified interior through eddies and lateral stirring motions.

In the abyss, far removed from the wind-driven upper ocean, local and basin-scale circulations are largely set by buoyancy forcing. Stommel's early concept of the abyssal circulation (Stommel et al., 1958) described isolated polar sources which fed dense water into deep western boundary currents. The model assumed a uniform upwelling in the ocean interior. As the source waters spread away from the boundaries, the upwelling 
drove a slow meridional poleward return flow. In the absence of uniform diapycnal mixing a different pattern emerges. Direct measurements of the Brazil Basin's deep circulation were undertaken in the 1990's as part of the WOCE program (Hogg and Owens, 1999). The multiyear float study found zonal flows dominant over meridional motions and no consistent poleward component to the circulation. Hogg and Owens suggest that the complex basin scale circulation is related to the weak mixing over the abyssal plain and the distribution of localized sources and sinks along the MidAtlantic Ridge.

St. Laurent et al. (2001) use an inverse model constrained by dissipation and hydrographic data to investigate the mixing-related circulation near a transverse fracture zone of the MidAtlantic Ridge. The topography of the region consists of a canyon, which shoals eastward toward the peaks of the Ridge and is bounded by crests on either side. The model solution describes flow above the crests moving westward and downward toward greater density, while the flow along deeper isopycnals heads eastward into the canyon, upwelling and feeding the westward flow. If the circulation is considered characteristic of the 20-30 fracture zones within the Brazil Basin, it accounts for the upwelling implied by the budget calculations.

The tracer component of BBTRE provides direct evidence of diapycnal transport and local circulation patterns (Ledwell et al., 2000). A chemical tracer was released over the western slope of the MidAtlantic Ridge and tracked by sampling surveys over a two year period. A portion of the tracer was drawn eastward. Its transport was downward toward greater density, deep into two adjacent canyons and then back toward lighter isopycnals. Its dispersion indicated enhanced mixing close to the bottom and over the rough topography. The tracer behavior was consistent with both the inverse model and the microstructure diffusivity calculations.

The following points about the role of mixing in ocean dynamics emerge from the early concepts of source-driven abyssal flows (Stommel et al., 1958) and advectivediffusive balance (Munk, 1966) and the multiple observational and modeling studies of water properties and circulation patterns: 
1. Diapycnal mixing is a necessary link in the meridional overturning circulation, restoring surface properties to abyssal waters and sustaining the observed property distributions in the world oceans.

2. Mixing is unevenly distributed, with weak mixing throughout the ocean interior and intensified mixing concentrated over rough topography.

3. The magnitude of the combined mixing appears sufficient to account for basinscale heat and mass budgets, but the observed distribution of salinity and temperature require the advection of mixed but nonturbulent waters from regions of local mixing.

4. Small scale mixing can drive mesoscale and basin scale circulations.

This thesis presents a laboratory study that focuses on one aspect of the larger picture, the process of fluid exchange between a localized patch of turbulent mixing and a quiescent body of rotating stratified fluid. Few oceanic observations exist of turbulent mixing and its direct interaction with ambient waters. The phenomena are complex and remote, and the scarcity of data makes it difficult to understand the underlying dynamics. In the laboratory, aspects of the process can be isolated and studied directly.

The experiments presented here are performed in a rotating tank filled with linearly stratified salt water. Turbulence is mechanically imposed by a single oscillating bar of finite length placed at middepth along a section of the tank wall. Stratification and rotation confine the active turbulence to the immediate vicinity of the bar, creating a localized mixing region within the tank. The turbulent zone quickly reaches a steady state height, and the layer of mixed fluid collapses under the force of gravity, exporting mixed, but no longer turbulent, fluid into the interior along neutral density surfaces.

In this simplified and controlled laboratory setting it is possible to examine the entrainment of ambient fluid into the turbulent zone, the export of mixed fluid, its properties, volume and distribution, and the overall flow field associated with localized mixing. These characteristics are described and quantified as functions of the external experimental parameters: the Coriolis frequency,

$$
f=2 \Omega,
$$


where $\Omega$ is the tank rotation rate; the buoyancy frequency, $N$, defined by

$$
N^{2}=-\frac{g}{\rho_{o}} \frac{\partial \rho}{\partial z},
$$

where $g$ is the acceleration due to gravity, $\rho_{o}$ is the reference density, and $\rho(z)$ is the ambient density; the bar oscillation frequency, $\omega$; and the horizontal extent of the mixing region given by the bar length, $L_{B}$. Scaling laws derived from the relationships between the external parameters and the properties of the mixed fluid are used to interpret the underlying physical processes. The intent is understand the role of stratification and rotation in the formation and distribution of turbulently mixed fluid.

The laboratory experiments also explore the effects produced by topographic variations in the mixing region. Two simple geometries are employed, mixing at a vertical boundary and laterally confined mixing. A comparison of the two configurations suggests that topographic features play a significant role in the formation and distribution of mixed water.

Chapter 2 discusses the choice of the single oscillating bar as the mixing mechanism and reviews previous laboratory studies of mechanically-induced turbulence. The review emphasizes the parameterization of grid-generated turbulent properties and relates these properties to the inhibition of turbulence by stratification or rotation. The length scales and entrainment laws developed in the previous studies provide a basis for interpreting results in the present experiments. This chapter also discusses the distinguishing features of the present experimental study in the context of previous laboratory investigations that combine the effects of stratification and rotation.

Chapter 3 describes the laboratory apparatus and methods, and specifies the range of external variables used in the current research. The chapter includes an experimental study of turbulence generated by the single bar, develops a parameterization of the associated turbulent properties and derives bar-specific length scales for use in evaluating the laboratory observations. 
Chapter 4 presents the initial set of experiments where the mixing bar is placed at a vertical boundary and exposed on three sides to the surrounding water. In these experiments, a single mixed layer of uniform thickness and finite width moves out from the bar directly into the interior, until its coherent advance gives way to instability and eddy shedding. Surprisingly, the emerging intrusion does not turn to the right and form a cyclonic boundary current. Instead, the exported fluid appears to be constrained by entrainment-driven flows approaching both sides of the turbulent region. The production of mixed fluid at the bar and its distribution in the basin are shown to be functions of both rotation and stratification. The dependence on rotation, in particular, appears related to the horizontal circulation patterns and the lateral entrainment process.

Chapter 5 presents a second set of experiments that explores the dependence on lateral entrainment by confining the sides of the mixing bar between two parallel walls, which form a channel open to the basin at the far end. As the mixed fluid moves out from the bar, a small percentage enters a boundary current along the right-hand wall, but the bulk of the mixed fluid forms a recirculating cell at the base of the channel. This restricts lateral entrainment of unmixed fluid into the turbulent zone, and curtails the ongoing production of mixed fluid. In the confined case, the production of mixed fluid decays with time.

The concluding chapter discusses the unique aspects of the laboratory experiments and summarizes their contributions to the study of turbulent mixing and their implications for localized mixing along ocean boundaries. 


\section{Chapter 2}

\section{A Review of Laboratory Experiments with Mechanically-Induced Turbulence}

\subsection{Introduction}

The spatial and temporal distribution of dissipation rates observed in the Brazil Basin and over Pacific seamounts suggests that the energy for local turbulent mixing comes from internal waves that are generated by tidal flow over rough topography, propagate upward and develop instabilities (Ledwell et al., 2000). Localized mixing in the ocean also arises from other causes including boundary stress, shear flow and double diffusion. In the laboratory it is difficult to produce sustained controlled mixing through similar processes. Since the goal of the present research is to examine the effects, rather than the source, of localized mixing, the experiments use mechanical means to impose turbulence. The mixing mechanism is a horizontally oriented, oscillating bar.

Laboratory mixing studies, employing the mechanism of internal wave breaking, have focussed primarily on the characteristics of the wave-induced turbulence rather than on interactions between local turbulence and the ambient water. Ivey and Nokes (1989) and Taylor (1993) investigated turbulence induced by a train of uniformly distributed waves, while De Silva et al. (1997) studied an isolated patch of turbulence at the boundary. 
These experiments used a paddle to generate waves incident on a sloping boundary at the far end of a long narrow tank. The breaking waves created turbulence at the boundary, and when the turbulence was energetic enough a mixed layer was established and horizontal intrusions of mixed fluid were exported into the interior. However, the experiments also showed that shoaling waves had a cycle of generation and decay, and that the dissipation rate of kinetic energy was highly variable. The experiments provided insight into the temporal and spatial variability of wave-induced mixing.

Mechanically-induced mixing is not designed to capture this variablity. It is used in the present experiments to produce a local region of sustained, highly energetic turbulence at the vertical boundary of a much larger body of undisturbed water. The laboratory setup is kept simple in order to isolate the interaction between the mixing region and the ambient fluid.

The experiments are conducted in a rotating tank filled with linearly stratified salt water, and turbulence is induced by a single oscillating bar of finite length placed at middepth along a section of the tank wall. The oscillations generate overturning motions which are confined to the immediate vicinity of the bar by the ambient stratification and background rotation. The experiments investigate the exchange between the turbulent region and the surrounding water, and examine the production of mixed fluid and the circulation patterns set up through the entrainment of ambient water and the distribution of mixed fluid in the interior

There is a large body of literature on mechanically induced turbulence. This chapter draws on that literature to provide a background for the current experiments. It presents some of the fundamental results on the structure of grid-generated turbulence and on the effects of rotation and stratification on entrainment and mixing. It also reviews three laboratory studies that consider specific oceanographic examples. The selected studies cover vertical boundary mixing, cross-frontal exchange and mixing in a channel and consider the larger scale effects of localized mixing in a rotating, stratified basin. 


\subsection{Properties of grid-generated turbulence}

The experiments of Hopfinger and Toly (1976) studied the spatial decay of mechanicallyinduced turbulence in a homogeneous fluid and related turbulent velocity and length scales to the geometry and motion of the mixing mechanism. The mixer was a vertically oscillating grid of square bars that spanned a horizontal plane at middepth in a rectangular tank. The schematics of the experimental setup are sketched in Figure 2.1. Direct measurements of the rms turbulent velocities $u_{t}$ and the integral length scales $l_{t}$ demonstrated that the oscillating grid produced nearly isotropic turbulence with high Reynolds and Peclet numbers.

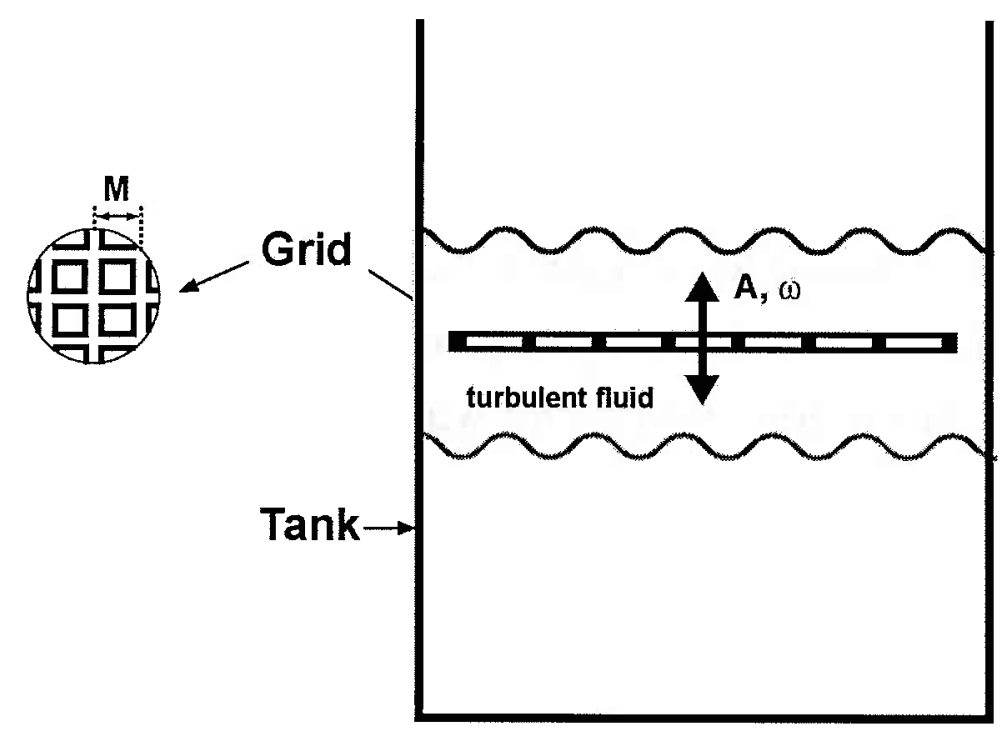

Figure 2.1: A schematic diagram of the experimental setup used by Hopfinger and Toly (1976). A horizontal grid of intersecting square bars is located at middepth in a square tank. The grid has a mesh spacing, $M$, and an oscillates vertically with amplitude, $A$, and frequency, $\omega$. 
The turbulent Reynolds number, a ratio of inertial to viscous force, is defined by

$$
R e_{t} \equiv \frac{u_{t} l_{t}}{v}
$$

where $v$ is the kinemtaic viscosity of water. The Peclet number is defined by

$$
P e_{t} \equiv \frac{u_{t} l_{t}}{K_{S}},
$$

where $\kappa_{S}$ is the molecular diffusivity of salt. The laboratory measurements showed that turbulent velocities decayed with distance $d$ from the grid, $u_{t} \sim d^{-1}$, and that length scales $l_{t}$ increased linearly with distance.

$$
l_{t}=\mathcal{B} d
$$

where $\mathcal{B}$ is an empirically determined constant. As a consequence, $R e_{t}$ and $P e_{t}$ were independent of location and approximately constant. In the Hopfinger and Toly experiments $R e_{t}=O\left(10^{2}-10^{3}\right)$ and $P e_{t}=O\left(10^{5}-10^{6}\right)$. The high values indicated that, to the first order, viscous and diffusive effects could be ignored in the grid-induced turbulent flows.

The experiments also established the dependence of turbulent velocity on properties of the mixing mechanism. Mixing was thought to result from wake-wake interaction of flow between the grid bars. Therefore the grid geometry was specified by the mesh spacing $M$ rather than the bar size. Grid motion was characterized by the oscillation frequency $\omega$, and amplitude, $A=S / 2$, where $S$ was the stroke. Velocity was related to the grid properties and distance $d$ by

$$
u_{t}=C \frac{A^{3 / 2} M^{1 / 2} \omega}{d}
$$

with the constant of proportionality $C$ empirically determined.

Based on the experimental results of Hopfinger and Toly (1976), showing $u_{t} \propto \omega / d$ and $l_{t} \propto d$, Long (1978a, 1978b) developed a mixing length model for grid-induced turbulence and defined a constant $K$, termed the grid action, 


$$
K=u_{t} d
$$

The Long model assumes that $d$ is the relevant length scale for specifying grid-induced turbulent flow and that $t \sim d / u_{t}$ is the relevant time scale. Since $u_{t} \sim d^{-1}$, this implies that the turbulent region will advance as $d-t^{1 / 2}$. The $\frac{1}{2}$ power time dependence is verified in the homogeneous turbulence experiments of $\mathrm{E}$ and Hopfinger (1986).

The parameter $K$ incorporates the mixing characteristics of the grid. For the Hopfinger and Toly mechanism (2.4) and (2.5) give the grid action,

$$
K_{H T}=C A^{3 / 2} M^{1 / 2} \omega \text {. }
$$

$K$ will be used in the remainder of this chapter to represent the mixing characteristics of various mechanisms.

\subsection{Turbulence and stratification}

\section{3.a Buoyant inhibition of turbulence}

When turbulence occurs in stably stratified fluids, buoyancy forces tend to inhibit vertical motion. The ratio of buoyant to inertial forces is given by the nondimensional turbulent Richardson number,

$$
R i_{t} \equiv \frac{N^{2} l_{t}^{2}}{u_{t}^{2}},
$$

with $u_{t}$ and $l_{t}$ evaluated locally at the interface between the turbulent mixed layer and the nonturbulent stratified fluid. Close to the grid the mechanically induced turbulence has small length scales and large velocity scales. As the edge of the mixed region moves away from the grid, the interfacial value of $u_{t}^{2} / l_{t}^{2}$ diminishes.

Ozmidov (1965) described the length scale $L_{O}$ at which buoyancy forces equal inertial forces,

$$
L_{O}=\left(\frac{\varepsilon}{N^{3}}\right)^{1 / 2},
$$

where $\varepsilon$, the dissipation rate of turbulent kinetic energy, is given by 


$$
\varepsilon=\frac{u_{t}^{3}}{l_{t}} .
$$

Equation (2.8) implies that stratification has little effect on turbulence with length scales $l_{t}<L_{O}$. When $l_{t}=L_{O}$, the turbulent length scale represents the maximum size of turbulent overturning in the presence of stable stratification. This limit is described by an internal turbulent Froude number of order one, where the Froude number is defined by

$$
F r_{t} \equiv \frac{u_{t}}{N l_{t}}
$$

For $F r_{t}=O(1), l_{t}=\frac{u_{t}}{N}$, and $l_{t}=L_{O}$. Buoyancy is expected to suppress overturning at a distance from the grid where $F r_{t} \leq 1$. The critical distance, $d_{N}$, is derived from the definition (2.10) and the grid parameterizations of turbulent properties, $l_{t}(2.3)$ and $u_{t}$ (2.5),

$$
d_{N} \sim\left(\frac{K}{N}\right)^{1 / 2}
$$

Fernando (1988), using an apparatus similar to the one shown in Figure 2.1, generated turbulence in a linearly stratified fluid. As predicted by the Long model, the height $h$ of the mixed layer grew as $h \sim(K t)^{1 / 2}$ and reached an equilibrium level,

$$
h_{N} \sim\left(\frac{K}{N}\right)^{1 / 2} \text {. }
$$

The height, $h_{N} \sim d_{N}$, was set in a time,

$$
t \sim N^{-1}
$$

The laboratory studies of Maxworthy and Monismith (1988), Davies et al. (1991), and De Silva and Fernando (1998) also noted the initial rapid growth period, $t \leq N^{-1}$, establishing a mixed layer height, $h \sim(K / N)^{1 / 2}$ at $t \sim N^{-1}$.

At the critical height, buoyancy forces arrest turbulent overturning and a sharp density interface forms. Growth of the mixed layer through turbulent engulfment of unmixed 
fluid is greatly inhibited, and further entrainment across the interface proceeds at a much reduced rate.

\section{3.b Mixed layer deepening and the entrainment hypothesis}

The entrainment hypothesis (Turner, 1986) states that the inflow velocity $u_{e}$ at the edge of a turbulent region is proportional to the local rms turbulent velocity $u_{t}$.

$$
u_{e}=\mathcal{D} u_{t},
$$

where $\mathcal{D}$ is a constant of proportionality, and the entrainment velocity $u_{e}$ is defined by the change in height of the turbulent layer, $d h / d t$. For steadily forced turbulence with high Reynolds (2.1) and Peclet numbers (2.2), the constant of proportionality, $\mathcal{D}$, is expected to depend only on $l_{t}, u_{t}$ and $N$, and can be written as a function of the turbulent Richardson number (2.7).

The experimental results of Turner (1973), Hopfinger and Toly (1976), and E and Hopfinger (1986) support the choice of the Richardson number as the parameter specifying the relative entrainment rate, $u_{e} / u_{t}$. These studies obtained an entrainment law of the form,

$$
\frac{u_{e}}{u_{t}} \sim R i^{-3 / 2}
$$

Linden (1973) suggested a physical explanation, a model of turbulent eddies impinging on the interface between the turbulent and stratified fluid with mixing occurring at the buoyancy time scale during the recoil of the interface. He applied the model to observations of vortex rings interacting with a density interface and calculated a volume rate of entrainment that agreed with the $-\frac{3}{2}$ power law.

The $-\frac{3}{2}$ power law implies that after the critical height (2.12) is reached, further entrainment across the density interface will go as $\mathrm{t}^{1 / 8}$. This result is demonstrated below following Maxworthy and Monismith (1988). From (2.7) the entrainment hypothesis (2.15) can be written as 


$$
\frac{d h}{d t} \sim u_{t}\left(\frac{u_{t}^{3}}{N^{3} l_{t}^{3}}\right) .
$$

At the interface $l_{t} \sim h(2.3), u_{t} \sim K / h(2.5)$, thus

$$
\frac{d h}{d t} \sim K^{4} N^{-3} h^{-7}
$$

The following transformations of variables, $h$ and $t$,

$$
\eta=\frac{h}{(K / N)^{1 / 2}}
$$

and

$$
\tau=N t
$$

give

$$
\frac{d \eta}{d \tau} \sim \eta^{-7}
$$

For $\eta=0$ at $t=0$, integration of equation (2.20) shows

$$
\eta \sim \tau^{1 / 8}
$$

Once the mixed layer reaches the critical height, further growth is given by

$$
h \sim\left(\frac{K}{N}\right)^{1 / 2}(N t)^{1 / 8}
$$

Not all experiments demonstrate the same growth rates. For instance, the experiments of Fernando (1988) indicated a longterm growth rate, $h-t^{1 / 9}$. This result corresponded to an entrainment relation of the form $\frac{u_{e}}{u_{t}} \sim R i^{-7 / 4}$ and supported the Long (1978a) model of intermittent wave breaking within the interfacial layer. Growth rates of either $t^{1 / 8}$ or $t^{1 / 9}$ are small. The intention here is not to choose one power law over another but to lay the groundwork for evaluating vertical entrainment in the more complicated setup of localized boundary mixing. 


\section{3.c Localized mixing and mixed layer deepening}

The experiments described above, in Sections 2.3.a and 2.3.b, examined uniform mixing over an entire horizontal plane within a tank. They considered one-dimensional vertical entrainment without any significant contribution from lateral advection. When the generation of turbulence is spatially inhomogeneous, the effects of buoyancy are seen not only in the arrest of vertical overturning scales but also in the collapse of threedimensional turbulence into lateral intrusions of mixed fluid. The two studies discussed next investigated the dynamics of mixed layer thickening in the presence of lateral exchanges between the turbulent region and the quiescent linearly stratified interior.

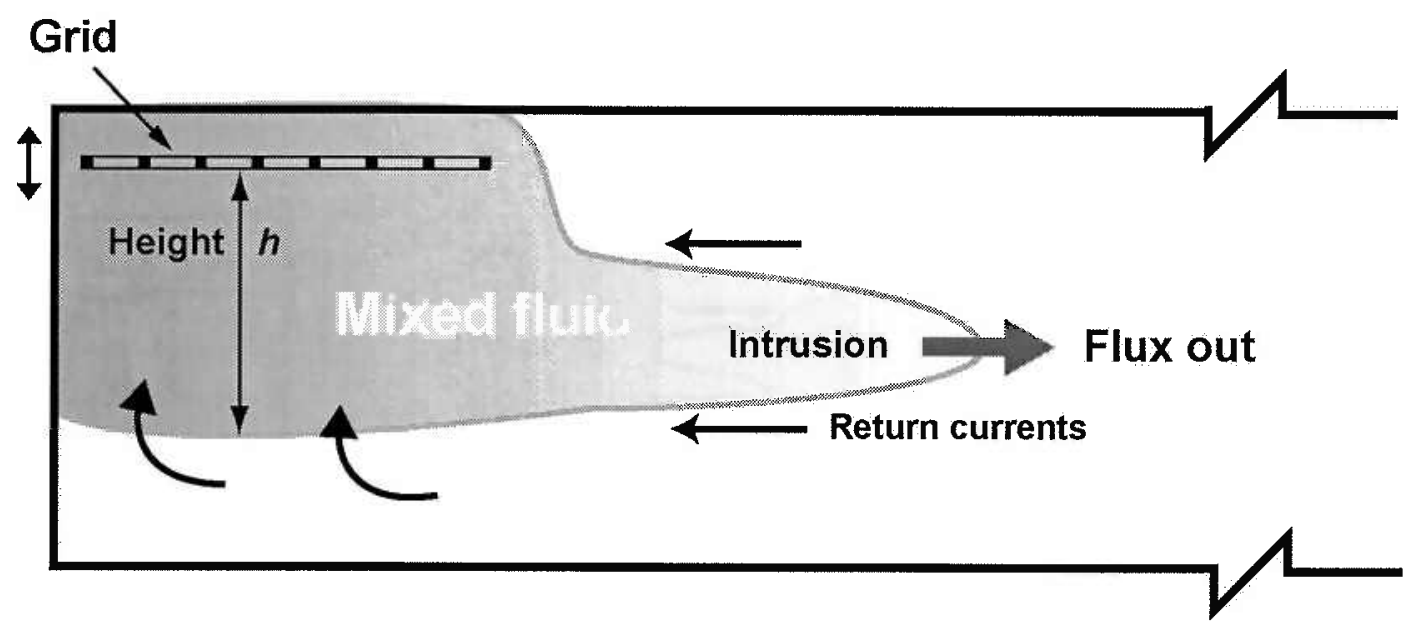

Figure 2.2: Sketch of the Maxworthy and Monismith (1988) experiment. The vertically oscillating grid is located near the upper surface at one end of a long rectangular tank. The mixed layer grows vertically beneath the grid and then collapses into the interior of the tank.

Maxworthy and Monismith (1988) set up an experiment using a horizontal grid mounted near the surface at one end of a long rectangular tank. The mixing region was bounded by the tank walls on three sides and open to the interior on the fourth as shown in Figure 2.2. The experiment identified three phases in the vertical growth of the mixed 
patch under the grid. The initial phase of downward growth went as $\mathrm{t}^{1 / 2}$. When the patch reached a critical height, (2.12), the mixed fluid collapsed into the interior. The start of intrusive flow was generally accompanied by a period of zero vertical growth.

Maxworthy and Monismith attributed the constant patch depth of phase two to a balance between horizontal outflow and vertical entrainment. The patch resumed growth when the intrusion was blocked by the far boundary of the tank. The mixed layer, both patch and intrusion, thickened throughout the tank. In the final phase,

$$
h \sim\left(\frac{K}{N}\right)^{1 / 2}\left(\frac{L_{G}}{L_{T}}\right)^{1 / 8}(N t)^{1 / 8},
$$

where $L_{G}$ is the grid length and $L_{T}$ is the tank length. The growth was comparable to the one-dimensional calculation (2.22), modified by a weak dependence on $L_{G} / L_{T}$.

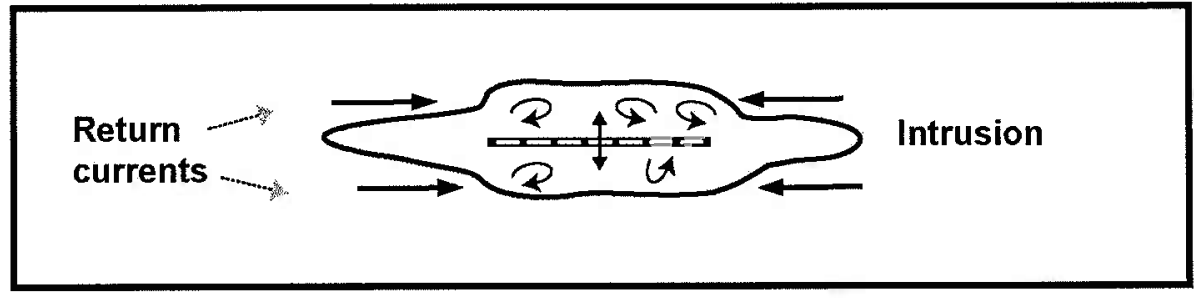

Front view

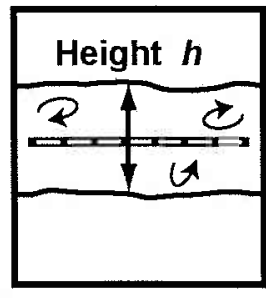

Side view

Figure 2.3: A diagram of the De Silva and Fernando (1998) experiment. A horizontally oriented, vertically oscillating grid is placed at middepth across the midsection of a long rectangular tank. Intrusions of mixed fluid spread out from both sides of the central patch. Return currents are observed at the upper and lower edges of the patch.

De Silva and Fernando (1998) conducted a similar experiment, but placed the grid at middepth across the midsection of the tank. The apparatus and observed flow patterns are shown in Figure 2.3. As in the Maxworthy and Monismith (1988) experiment, an initial period of rapid vertical growth at the grid was followed by the emergence of 
intrusions and the maintenance of a constant patch thickness. Once the intrusive flows were blocked by the far walls of the tank, thickening of the patch resumed. For a brief period its growth showed a time dependence comparable to the $t^{1 / 8}$ and $t^{1 / 9}$ regimes observed in earlier one-dimensional experiments. However, as the lateral intrusions on either side of the patch filled out, the patch and side layers deepened simultaneously at a more rapid rate. The longterm growth regime showed $h \sim t^{1 / 5}$.

De Silva and Fernando suggested that horizontal rather than vertical entrainment controlled mixed layer growth. During the emergence of the intrusions they observed return currents entering the mixing region at the upper and lower edges of the patch. Furthermore, they tested the role of vertical entrainment by varying the grid length by a factor of two and found no change in growth rates. They proposed a model of mixed layer growth based on return currents injecting fluid into the turbulent patch over a distance proportional to the scale height of the mixed layer (2.12). They hypothesized that mixing occurs locally due to shear instabilities in the injected flow and that the conversion of kinetic to potential energy is proportional to the ratio of the injection length to the tank length, $\left(\frac{K}{N}\right)^{1 / 2} / L_{T}$. At long time scales the model predicted growth proportional to $t^{1 / 5}$,

$$
h \sim\left(\frac{K}{N}\right)^{1 / 2}\left(\frac{(K / N)^{1 / 2}}{L_{T}}\right)^{1 / 5}(N t)^{1 / 5} .
$$

The model provided a good fit to the laboratory data.

\section{3.d Vertical boundary mixing}

A series of experiments with vertical boundary mixing focussed on the horizontal propagation of turbulence and the dimensions of the nonturbulent lateral intrusions of mixed fluid. These studies are reviewed here, both to present their results and to outline a method for inferring local turbulent quantities, $u_{t}$ and $l_{t}$, in the absence of direct measurements. 
The studies of Ivey and Corcos (1982), Thorpe (1982), and Browand and Hopfinger (1985) used vertically oriented planar grids to generate a wall of turbulence in a linearly stratified fluid. Figure 2.4 shows the Browand and Hopfinger apparatus with a horizontally oscillating grid of intersecting bars. In the other two experiments the grids differed in bar pattern, direction of motion and location, but all spanned a vertical plane through the interior of the tank. In all three sets of experiments, turbulence was confined to the immediate vicinity of the grid and multiple horizontal layers of mixed but nonturbulent fluid moved from the mixing region into the interior.

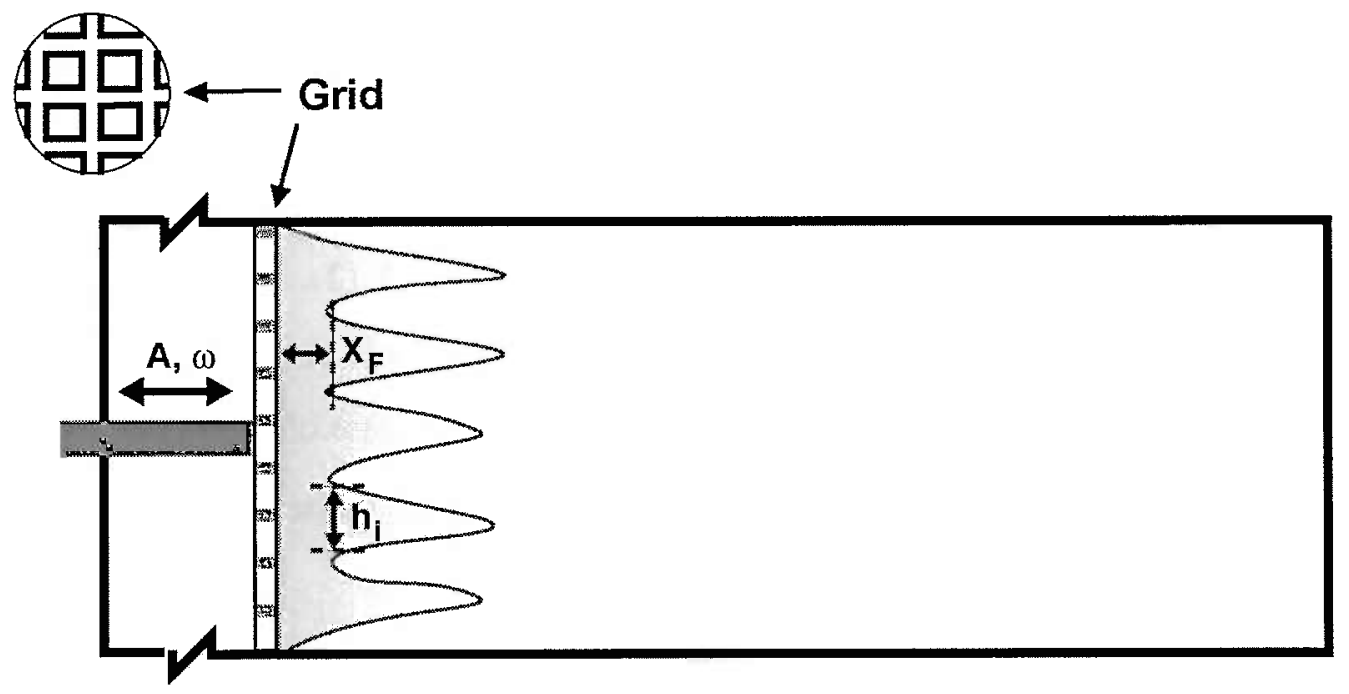

Figure 2.4: $\quad$ A sketch of the apparatus used by Browand and Hopfinger (1985). The rectangular tank is filled with linearly stratified salt water. The vertically oriented planar grid of intersecting square bars oscillates hroizontally, with amplitude $A$ and frequency $\omega$. A vertical layer of turbulence develops next to the grid, and multiple horizontal intrusions of mixed, but no longer turbulent, fluid move into the interior of the tank. $X_{F}$ marks the position of the turbulent front; $h_{\mathrm{i}}$ is the height of the emerging intrusion.

Ivey and Corcos were interested in vertical mass flux in the presence of boundary mixing. The experiment used a vertically oscillating plate with evenly spaced horizontal bars to generate turbulence. Figure 2.5, Section A-A sketches a similar grid. Ivey and Corcos investigated the advance and arrest of the turbulent front and the emergence of intrusions into the interior, as well as the changes in the overall density distribution. They 
concluded that the intrusions themselves contributed little to vertical mass transport and that vertical mixing was restricted to the turbulent zone. In addition, they found that the width of the turbulent zone and the height of the intrusions were linearly related to $\omega A / N$, where $N$ is the initial stratification and $\omega A$ is the forcing velocity with $\omega$ and $A$ the grid frequency and amplitude, respectively. They interpreted the intrusion height $h_{i}$ as a measure of the gain in potential energy due to the conversion of a constant fraction of initial kinetic energy, $N h_{i} \propto \omega A$.

Thorpe (1982) assumed that the layered collapse of a horizontally propagating turbulent front was related to the buoyant suppression of vertical overturning, but he noted a close correspondence in the Ivey and Corcos experiment between intrusion heights and the vertical spacing of the horizontal grid bars. To explore the layering dynamics more fully, Thorpe conducted a boundary mixing experiment using a distinctly different grid, one constructed of vertical bars, oscillated horizontally. At the first appearance of intrusive flows, Thorpe measured the position of the turbulent front $x_{F}$ and the height of the intrusions $h_{i}$. He equated the measurements, respectively, with $d_{N}$ (2.11), the critical distance for the onset of buoyant suppression, and $l_{t}$, the turbuent length scale evaluated at $d_{N}$. The equation for $d_{N}$ was based on the velocity and length scale parameterizations of Hopfinger and Toly, (2.3) and (2.4), with appropriate gridspecific values for $A, M$ and $\omega$, and on a constant of proportionality adopted from Dickey and Mellor (1980). Dickey and Mellor studied structural change from turbulence to internal waves in the wake beneath a raised grid. They identified turbulent collapse with a constant value of the ratio of the buoyancy period $N^{-1}$ to the intrinsic time scale of the turbulence $l_{t} / u_{t}$, that is, with a turbulent Froude number (2.10),

$$
F r_{t}=\mathcal{E}=0.27 .
$$

Thorpe used (2.25) in defining the critical distance for his experiment,

$$
d_{N}=\frac{1}{\mathcal{E}^{1 / 2}}\left(\frac{C^{1 / 2}}{\mathcal{B}^{1 / 2}}\right) A^{3 / 4} M^{1 / 4}\left(\frac{\omega}{N}\right)^{1 / 2}
$$


By (2.3) and (2.26) the turbulent length scale at $d=d_{N}$ is given by

$$
\left.l_{t}\right|_{d=d_{N}}=\frac{1}{\mathcal{E}^{1 / 2}}\left(\mathcal{B}^{1 / 2} C^{1 / 2}\right) A^{3 / 4} M^{1 / 4}\left(\frac{\omega}{N}\right)^{1 / 2} .
$$

The data for the frontal position $x_{F}$ and the intrusion height $h_{i}$ demonstrated good straight line fits with $d_{N}$ and $\left.l_{t}\right|_{d_{N}}$, respectively, and specified values for the constants $C$ and $\mathcal{B}$.

Thorpe applied the same methodolgy to the Ivey and Corcos experiment and found a good fit to the revised scaling for their data as well. In a later experiment on vertical boundary mixing in the presence of rotation, Ivey (1987) acknowledged the Thorpe analysis and adopted a version of the Hopfinger-Toly (1976) parameterization. The Ivey and Corcos (1982) and Thorpe (1982) experiments both confirmed the general applicability of the $(K / N)^{1 / 2}$ scaling where $K=u_{t} d(2.5)$ and $u_{t}$ is specified by grid properties. The results suggested that the buoyant suppression of turbulent overturning affects both the horizontal and vertical limits of the turbulent zone.

Additional confirmation was provided by the laboratory study of Browand and Hopfinger (1985). Their experimental apparatus (shown in Figure 2.4) employed the Hopfinger-Toly grid. The Browand-Hopfinger experiment explicitly examined the relationship between the Ozmidov scale (2.8) and the scales of the turbulent front and lateral intrusions. At the location where the turbulent front differentiated into intruding layers, it was assumed that the turbulent length scale would be a constant multiple of the Ozmidov scale $L_{O}$,

$$
L_{O}=\left.\mathcal{F} l_{t}\right|_{d=d_{N}} .
$$

Predictions for the critical distance and the turbulent length scale followed from the Ozmidov scaling, (2.8) and (2.9), and the Hopfinger-Toly parameterization of turbulent properties, (2.3) and (2.4),

$$
d_{N}=\frac{1}{\mathcal{F}^{1 / 3}}\left(\frac{C^{1 / 2}}{\mathcal{B}^{1 / 2}}\right) A^{3 / 4} M^{1 / 4}\left(\frac{\omega}{N}\right)^{1 / 2}
$$




$$
\left.l_{t}\right|_{d=d_{N}}=\frac{1}{\mathcal{F}^{1 / 3}}\left(\mathcal{B}^{1 / 2} C^{1 / 2}\right) A^{3 / 4} M^{1 / 4}\left(\frac{\omega}{N}\right)^{1 / 2} .
$$

The grid constants, $C$ and $\mathcal{B}$, had been previously established by Hopfinger and Toly, but the constant of proportionality $\mathcal{F}$ was an unknown. Browand and Hopfinger plotted the measured position of the turbulent front $x_{F}$ versus $d_{N}(2.29)$ and calculated the value of $\mathcal{F}$ from the straight line fit. The factor, $\mathcal{F}^{-1 / 3}$, was dynamically equivalent to the constant, $\mathcal{E}^{-1 / 2}$, used by Thorpe in defining the critical distance $d_{N}(2.26)$. From the definition of the Ozmidov scale (2.8) and equation $(2.28), \mathcal{F}^{2 / 3}$ can be identified as a Froude number (2.10),

$$
\mathcal{F}^{2 / 3}=\frac{u_{t}}{l_{t} N} \equiv F r_{t}
$$

The empirically determined value, $\mathcal{F}^{2 / 3}=0.26$, was found to be nearly identical to $\mathcal{E}=0.27(2.25)$. Browand and Hopfinger also measured the height of the intrusion $h_{i}$ at the edge of the front and plotted it versus the calculated value of $l_{t}(2.30)$. They found that the measured heights matched the predicted values, with $h_{i}=1.1 l_{t}$. Browand and Hopfinger arrived at basically the same results as Thorpe but by a different route.

In summary, experiments with the buoyant suppression of grid-induced turbulence have shown that at a sufficient distance from the source of the turbulence, $d_{N}(2.11)$, buoyancy will suppress vertical overturning. The vertical growth of the mixed layer will be dramatically reduced and the horizontal motions will change from three-dimensional turbulence to gravity-driven laminar intrusions.

\subsection{Turbulence and rotation}

\section{4.a Rotational inhibition of turbulence}

Rotation imposes constraints on three-dimensional turbulence. Where buoyancy suppresses vertical motion, rotation mainly affects motion perpendicular to the axis of 
rotation (Jacquin et al., 1990). Dickinson and Long (1983) and Hopfinger, Browand and Gagne (1982) examined rotational inhibition of turbulence in unstratified experiments that were analogous to the vertical boundary mixing experiments of Ivey and Corcos (1982), Thorpe (1982), and Browand and Hopfinger (1985). The stratified experiments used vertically oriented mixing grids to generate turbulence. The three-dimensional turbulence was restricted to the vicinity of the grids and the horizontal flow of mixed fluid into the interior was laminar and along neutral density surfaces. In the rotating experiments, vertically oscillating horizontal grids were used to generate horizontal planes of three dimensional turbulent motion. The basic setups were cylindrical versions of the tank sketched in Figure 2.1.

The analogy with stratified flow suggests that turbulent motions close to an oscillating grid will behave independently of rotation and propagate outward as $t^{1 / 2}$. The analogy also suggests that at a sufficient distance from the grid, rotation will suppress turbulent motion and the flow structure will change. Rotation is expected to exert an effect when the turbulent Rossby number,

$$
R o_{t} \equiv \frac{u_{t}}{f l_{t}},
$$

approaches unity from above. Here $f$ is the Coriolis frequency. The critical distance $d_{f}$, where $R o_{t}=O(1)$, is determined by the properties of grid-generated turbulence, (2.3) and (2.5),

$$
d_{f} \propto\left(\frac{K}{f}\right)^{1 / 2} \sim\left(\frac{\omega}{f}\right)^{1 / 2} .
$$

The rotating experiments showed that a turbulent front, formed at the horizontal grid, propagated vertically, parallel to the axis of rotation, with $d \sim(K t)^{1 / 2}$. At a distance proportional to $d_{f}(2.33)$, a dramatic change occurred from three-dimensional turbulence to two-dimensional rotationally dominated flows with strong vertical coherence. Dickinson and Long observed a transition to organized vertically oriented sheets of dye, resembling Taylor curtains. Hopfinger et al. described the emergence of concentrated 
vortices with axes parallel to the axis of rotation. The horizontal scale of the vortices corresponded to the local integral length scale $l_{t}$ at the critical height and were proportional to $(K / f)^{1 / 2}$. Hopfinger et al. found that the collapse of three-dimensional turbulence occurred at a Rossby number $R o_{t} \approx 0.20$, and they interpreted the observations of Dickinson and Long as indicating a transition value of $R o_{t} \approx 0.27$ to 0.31 .

Dickinson and Long (1983) also conducted a rotating tank experiment designed as an analogy to the one-dimensional stratified experiments. The one-dimensional stratified experiments imposed a horizontal plane of uniform mixing and examined the vertical evolution of turbulence. The rotating experiment imposed a cylinder of uniform mixing along the axis of rotation and examined the horizontal evolution of turbulence. Turbulence was generated by a vertically oscillating rod with evenly spaced O-rings, and turbulent velocity was characterized by the oscillation amplitude $A$ and frequency $\omega$, and as a function of distance $d$ from the rod: $u_{t} \sim \omega A^{2} / d$. Thus the grid action parameter for this mechanism was given by $K \sim \omega A^{2}$. Dickinson and Long showed data from only two experiments. The turbulent fronts initially advanced as $t^{1 / 2}$, and eventually established quasisteady positions. Dickinson and Long noted that the final radii were not proportional to $f^{-1 / 2}$ and thus did not conform to the predicted critical distance (2.33). However, the limited data do show the final radii inversely related to the Coriolis frequency. If the geometric effect of radial spreading is taken into account, they appear close to expected positions.

In general, the experiments of Dickinson and Long (1983) and Hopfinger et al. (1982) demonstrate that rotation affects turbulent structures in a homogeneous fluid. The following experiments explore the impact of rotation on turbulent mixing in a stratified fluid. 


\section{4.b Rotational effects on vertical entrainment}

In the absence of rotation, vertical entrainment across a density interface has been shown to be a function of the inverse Richardson number (2.7), related only to the turbulence quantities, $l_{t}^{2}$ and $u_{t}^{2}$, and the stratification $N^{2}$ (see Section 2.3.b). In the presence of rotation, entrainment rates are also a function of the Rossby number (2.32). Two laboratory studies (Maxworthy, 1986; Fleury et al., 1991) demonstrated that rotation reduced vertical mixing. The experiments both used vertically oscillating horizontal grids identical to that of Hopfinger et al. (1982). Turbulence was generated in one layer of a two-layer salt-stratified fluid and entrainment rates were calculated from the displacement of the interface over time. Three different conditions were studied: mixing with no rotation, mixing with rotation when the turbulent Rossby number (2.32) was larger than the critical value established by Hopfinger et al. (1982), $0.20<R o_{t} \leq O(1)$, and mixing in flows with $R o_{t}<0.20$.

Maxworthy noted that entrainment decreased with decreasing values of the Rossby number for $R o_{t}<1$, even though rotation was not expected to inhibit turbulent motions until $R o_{t} \approx 0.20$. He proposed a qualitative explanation based on the Linden (1973) model of turbulent eddies impinging on the density interface. He suggested that in the presence of rotation turbulent distortions of the interface induced inertial waves that radiated away and depleted the energy available for mixing.

In nonrotating flows the results of both Maxworthy and Fleury et al. indicated a $R i^{-3 / 2}$ dependence (2.15). Fleury et al. found an entrainment rate of the form,

$$
\frac{u_{e}}{u_{t}}=1.6 R i^{-3 / 2}
$$

In rotating flows, Fleury et al. confirmed Maxworthy's finding that entrainment rates were reduced for $0.20<R o_{t} \leq 1$, even though direct measurements showed turbulent velocities unaffected by rotation. Interface displacement spectra provided support for the Maxworthy hypothesis of inertial wave radiation into the unstirred layer. 
Fleury et al. correlated data from rotating flows covering $0.11 \leq R o_{t} \leq 1.3$ with the single entrainment law,

$$
\frac{u_{e}}{u_{t}}=0.5 R o R i^{-1}
$$

This relation is considered valid as long as the predicted entrainment is less than that given by the nonrotating law (2.34). It implies that the nonrotating law will apply to flows where $N / f>3.2$.

\subsection{Rotating, stratified experiments}

Additional information on the interplay of rotation, stratification and turbulence comes from experiments that were designed to look at the larger picture of localized mixing and its interaction with a rotating stratified environment. Ivey (1987) considered vertical boundary mixing in the presence of rotation, Thomas and Linden (1996) examined crossfrontal exchange, and Davies et al. (1991) studied the effects of mixing at one end of a rotating channel. The three experiments are described below..

The combined effects of rotation and stratification were introduced by Ivey (1987) in a two-layer adaptation of the Ivey and Corcos experiment (1982). In the rotating experiment, turbulence was imposed with a vertically oscillating cylindrical grid. Figure 2.5 shows a top view of the tank and a vertical section of the grid. The cylinder, ringed with evenly spaced horizontal bars, was an axisymmetric version of the Ivey and Corcos vertical plate. While the grid generated turbulence over the full depth of the tank, mixing was restricted to the density interface. Mixed fluid moved radially into the interior along the interface and developed into an anticyclonic boundary current. 

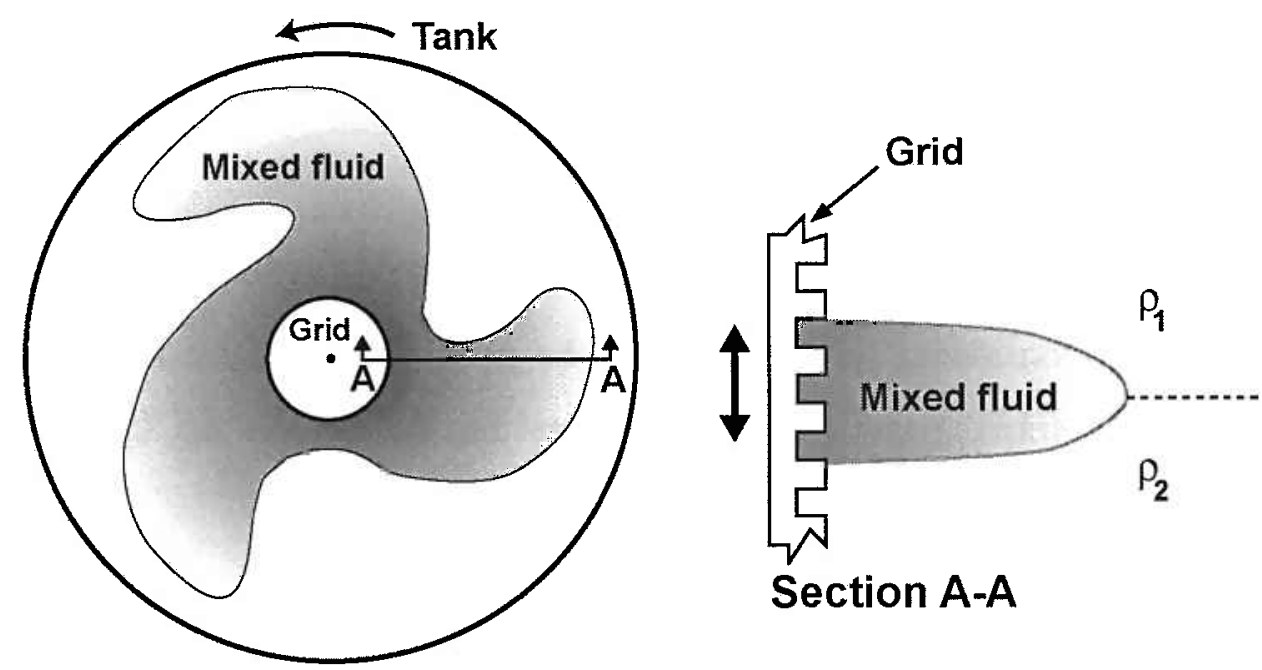

Top view

Figure 2.5: A schematic of the Ivey (1987) experiment. The top view of the tank shows the cylindrical grid located at the axis of rotation, and the mixed fluid spreading radially outward. Section A-A outlines the horizontal bars of the vertically oscillating grid, and depicts the mixed fluid emerging at the interface of two fluid layers of density $\rho_{1}$ and $\rho_{2}$.

Ivey adopted a slightly modified version of the Hopfinger-Toly (1976) parameterization of $l_{t}(2.3)$ and $u_{t}(2.4)$. The implied grid action $K$ for the cylindrical grid was defined by

$$
K=C A^{9 / 5} M^{1 / 5} \omega .
$$

Overall vertical buoyancy flux was estimated from conductivity profiles and evaluated in terms of the grid mixing parameters, the stratification and the rotation rate. The results were consistent with the earlier results of Ivey and Corcos, and Ivey concluded that vertical density flux was proportional to $\frac{\rho_{o}}{g}(K N)^{3 / 2}$ and independent of rotation. He noted, however, the role of rotation in the horizontal evolution of the density gradient. The boundary current, formed by the export of mixed fluid from the turbulent zone, widened with time and developed nonaxisymmetric disturbances when the current width 
grew to 2 to 5 times the Rossby deformation radius. The deformation radius was defined by

$$
R_{D}=\frac{\left(g^{\prime} h\right)^{1 / 2}}{f}
$$

where $g^{\prime}=\frac{g}{\rho_{o}} \Delta \rho$ represented the density anomaly between the propagating fluid and the environment, $h$ was the mixed layer depth, and $f$ was the Coriolis frequency. The disturbances ultimately grew into a chaotic eddy field that mixed fluid further into the basin interior. Ivey found that rotation determined the width of the coherent boundary current and the onset of instability, and thus affected the horizontal distribution of mixed fluid.

Thomas and Linden (1996) were interested in cross-front exchanges rather than boundary mixing, and designed an experiment with mixing across a horizontal density gradient. The mixing mechanism was a horizontally oriented, vertically oscillating flat circular grid, shown in Figure 2.6. It established a central column of intermediate density over the full depth of an initially two-layer system, and thus set up horizontal density gradients, i.e. fronts, between the central region and the interior. Mixed fluid was exported radially as in Ivey's experiment, and the spreading fluid displayed a pattern of developing eddies with an anticyclonic drift. Eddy diameters were of the order of deformation radius, consistent with the development of baroclinic instability at the front. Once the mixed layer reached the walls of the tank it began to thicken. Figure 2.6 sketches a vertical cross-section of the fully developed three-layer system. Thomas and Linden calculated cross front flows by estimating volume changes in the three layers. They expressed their results as an entrainment velocity,

$$
u_{e}=\beta \frac{\omega R^{2}}{2 r_{g}},
$$

where $r_{g}$ and $\omega$ are the grid radius and oscillation frequency, respectively, and $R$ is the radial position of the front. The nondimensional constant, $\beta$, is a function of the rotation rate and the density difference between the top and bottom layers, 


$$
\beta \propto\left(\frac{\omega}{f}\right)^{1 / 2}\left(\frac{g^{\prime}}{g}\right)^{1 / 3} .
$$

The empirical results indicated that entrainment increased with stratification and decreased with rotation. Note that the term $\left(\frac{\omega}{f}\right)^{1 / 2}$ is proportional to the length scale, (2.33), derived from a balance between turbulence and rotation. Thomas and Linden concluded that cross-frontal mixing, unlike vertical boundary mixing, was a result of horizontal entrainment and was governed by the rate at which stratified fluid could enter the mixing zone.

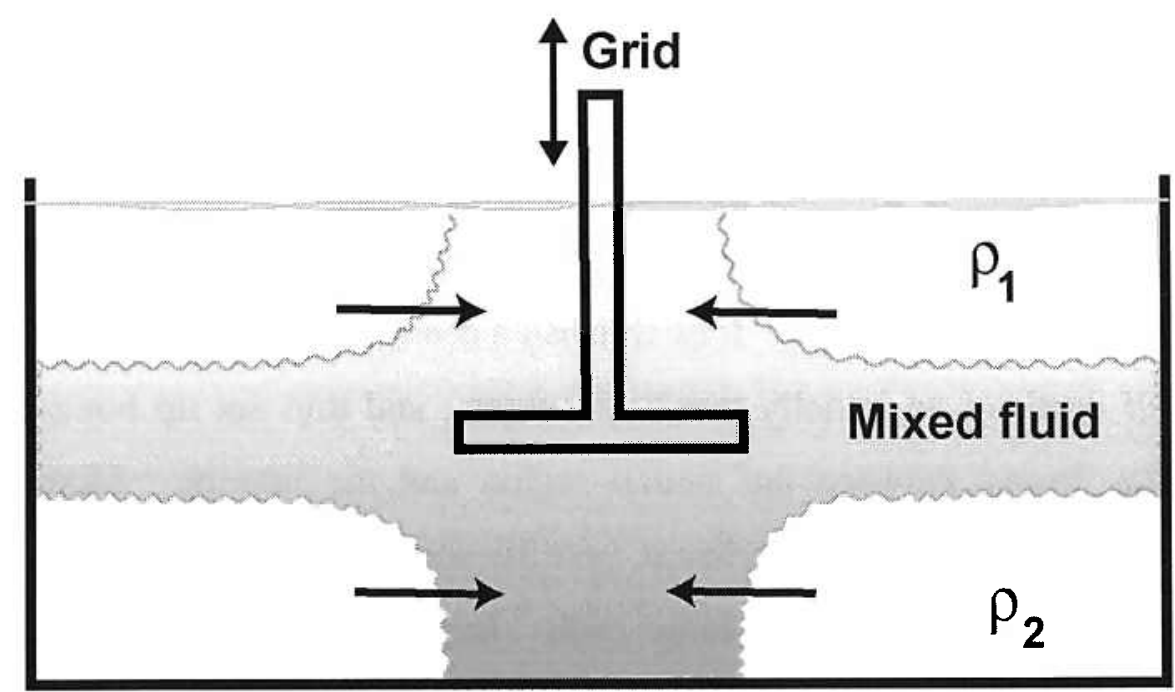

Figure 2.6: $\quad$ A sketch of the Thomas and Linden (1996) experiment with a two layer stratification in a rotating tank. The vertically oscillating flat circular grid produces a central column of intermediate density. The mixed fluid spreads radially outward between the two initial layers of density $\rho_{1}$ and $\rho_{2}$. The arrows indicate horizontal entrainment of unmixed fluid into the central mixing region.

It is interesting that turbulent entrainment of unmixed fluid in the Ivey and Thomas and Linden experiments appears to depend as much on the configuration of the mixing region as on variations in stratification and rotation. The structure of the mixing region in each case determined the possible pathways of fluid exchange and affected the roles of 
rotation and stratification in the production of mixed fluid. Davies et al. (1991) examined still another configuration for mixing in a rotating stratified fluid

Davies et al. imposed mixing at one end of a long rectangular tank filled with linearly stratified salt water. They used a vertically oscillating horizontal grid located at middepth, enclosed on three sides by the tank walls and extending $\frac{1}{7}$ the length of the tank. The arrangement was distinct from the full depth axisymmetric mixing mechanisms of Ivey and Thomas and Linden. Davies et al. could observe the vertical growth of the turbulent layer at the grid and the horizontal circulation setup by the outflow of mixed fluid. As in the nonrotating experiments of Maxworthy and Monismith (1988) and De Silva and Fernando (1998), the mixed layer quickly established a height, $h \sim(K / N)^{1 / 2}$, and exported mixed fluid into the interior. As in the rotating experiments of Ivey and Thomas and Linden, the outflow was deflected to the right forming a boundary current. However, since the outflow in this case was limited to a segment of the periphery, a compensatory return flow of unmixed fluid could develop along the left hand wall at the same level as the outflow. Over the long term, the boundary current followed the perimeter back to the source and the mixed layer at the grid resumed thickening. Further growth was an increasing function of rotation. Davies et al. concluded that, due to rotation, vertical entrainment was being augmented over long times by the lateral injection of already mixed fluid.

\subsection{Conclusion}

In the rotating stratified experiments that form the basis of the present study, mixing is imposed by a single bar located at middepth at the boundary of a much larger body of linearly stratified fluid. The configuration produces a small region of turbulent mixing open to both vertical and horizontal exchanges with the ambient fluid. In addition, it allows the outflow to evolve for some time without being blocked or otherwise constrained by the tank walls. The focus is on the production and distribution of mixed fluid, rather than on the long term dynamics of the closed system. 
In the initial body of experiments, that are described in Chapter 4, the bar is open on three sides to lateral exchanges with the surroundings. The exposed, highly limited nature of the patch of turbulent mixing distinguishes this study from earlier laboratory studies. A second set of experiments, described in Chapter 5, investigates the role of the horizontal exchange flows by cutting off access to the sides of the mixing region. The bar is enclosed between two walls extending from the bar into the center of the tank. Like the grid of Davies et al., the bar is now confined on three of its four sides at the end of a channel. However, the other end of the channel is open to a larger basin and the outflow of mixed fluid never circumnavigates the tank. Detailed descriptions of the open basin and channel experiments appear in Chapters 4 and 5. A further comparison of the experimental results to those of the Ivey, Thomas and Linden and Davies et al. is included in a discussion of the effects of the configuration of mixing region and basin on the interplay of mixing, rotation and stratification.

In general, the results of the current research are evaluated and interpreted in the context of the studies reviewed in this chapter. The foundation is laid in Chapter 3 which covers the laboratory setup and experimental design. Section 3.3 develops a parameterization of turbulence generated by the single bar mechanism. The model is based on the Hopfinger and Toly (1976) study of turbulent decay in homogeneous fluid (Section 2.2) and on the grid parameterizations of Thorpe (1982), Ivey and Corcos (1982) and Ivey (1987) in Section 2.3.d. Section 3.5 develops scaling arguments for evaluating the interaction of turbulent mixing and the ambient rotation and stratification. The length scales are derived from the theoretical and empirical relationships proposed by Ozmidov (1965), Fernando (1988), Dickinson and Long (1983), Hopfinger, Browand and Gagne (1982) and others reviewed in Section 2.3.a and 2.4.a. In Chapter 4 the evaluation of mixing with the single bar mechanism includes a discussion of the relative importance of vertical and horizontal entrainment and draws on the studies in Sections 2.3.b, 2.3.c and 2.4.a, such as those of Turner (1986) and Fleury et al. (1991). 


\section{Chapter 3}

\section{The Laboratory Setup}

\subsection{Introduction}

The laboratory experiments are designed to investigate the physics of localized mixing in a rotating, stratified fluid. Two experimental configurations are used. The first investigates the effects of a patch of mixing at a vertical boundary in an open basin. The second studies mixing at the closed end of a channel which opens into a basin at its other end. The configurations have been kept simple in order to isolate the interaction between the mixing region and the larger body of water. The main external parameters are the geometric characteristics of the tank, the imposed mixing, the fluid stratification, and the rotation rate of the system. The experiments relate these parameters, particularly variations in the buoyancy frequency $N$ and Coriolis frequency $f$, to the production and transport of mixed fluid and to the density and velocity distributions in the basin. The intent is to develop scaling laws that predict the observed flows and suggest the underlying dynamics.

Section 3.2 describes the experimental apparatus: the tank, the method of stratification, and the spin up of a rotating stratified fluid in the laboratory. Section 3.3 discusses the imposed mixing. The effects of the single bar mechanism are examined in a series of nonrotating experiments, and a model is proposed relating the induced turbulence to the bar properties. Section 3.4 describes the techniques used in visualizing and measuring the distribution of mixed fluid and circulation patterns in the tank. Section 
3.5 summarizes the range of parameter space, identifies the key nondimensional parameters characterizing the experiments, and defines the length scales used in analyzing the experimental results.

\subsection{Experimental apparatus}

Figure 3.1 sketches the laboratory apparatus, as viewed by an overhead video camera. It shows the $114 \times 114 \mathrm{~cm}$ square glass tank, with an interior circular plexiglass wall and an adjacent $45^{\circ}$ mirror. The wall prevents corner effects from complicating interior flows and the mirror provides a side view of the experiments. The entire apparatus is placed on a two meter diameter turntable. Both the table and the video camera, located five meters above the tank, rotate with angular frequency $\Omega=f / 2$ (counterclockwise when viewed from above).

The mixing mechanism is a vertically oscillating horizontal bar. Figure 3.1 shows the bar mounted at middepth on a support plate which is set into a flat section of the interior wall. The details of its operation are discussed in Section 3.3. In the unconfined vertical boundary mixing experiments, the bar is left exposed to the ambient fluid on three sides. In the channel experiments, the bar is laterally confined between two parallel plexiglass sheets. The sheets span the full depth of the water column and extend into the interior, three $\mathrm{cm}$ past the centerline of the tank. Figure 5.1 at the beginning of Chapter 5 sketches the channel configuration.

The tank is filled with linearly stratified water to a working depth of $26.5 \mathrm{~cm}$. The laboratory and the water are kept at a constant temperature. Stratification is accomplished through the two-reservoir method (Oster, 1965), which enables fresh and salt water to be combined into a stable, linear density gradient of predetermined strength. In these experiments increasingly salty water is introduced through a layer of sand into the bottom of the rotating tank over a two hour period. Passage through the sand helps to impart vorticity to the entering fluid. Prior to the initiation of mixing, additional time is allowed for the fluid to adjust to rotation. 


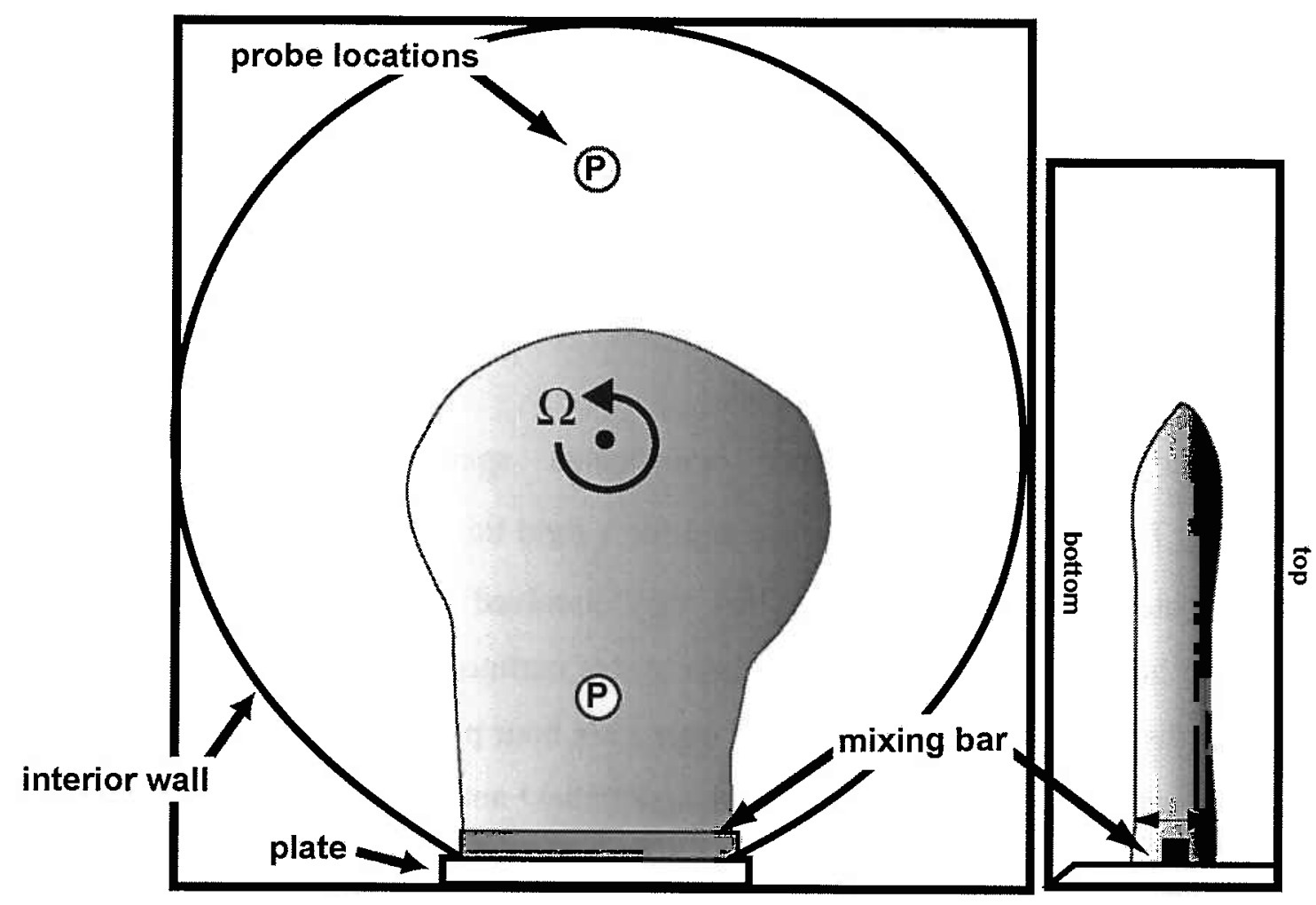

Figure 3.1: $\quad$ Sketch of the experimental apparatus, showing a top view of the $114 \times 114 \mathrm{~cm}$ square glass tank, the circular interior wall, the probe locations, and the mixing bar and support plate. To the right of the tank is a $45^{\circ}$ mirror giving a side view of the bar. The mixed fluid is shown moving directly out from the bar. Its area is measured from the top view; the height is measured from the mirror view at the site of the doubleheaded arrow.

For a stratified fluid spinup time $\tau$ is of the order $\left(H^{2} / v\right)$, where $H$ is the total depth and $v$ is the kinematic viscosity of water, $v=0.01 \mathrm{~cm}^{2} \mathrm{~s}^{-1}$. That implies that $\tau \approx 20 \mathrm{hr}$ for this tank. However, complete rigid body rotation cannot be achieved in a laboratory container, regardless of the elapsed time. The pressure surfaces of the fluid form paraboloids of revolution, and the density surfaces are out of parallel with them due to diffusion. The effective horizontal density gradient induces a relative flow, referred to as the Sweet-Eddington flow. Linear theory (Barcilon and Pedlosky, 1976) predicts an azimuthal thermal wind, antisymmetric about the middepth, becoming more cyclonic with height and increasing in magnitude with outward radial position. The diffusive 
processes which set up the thermal wind act slowly. Salt, the chosen stratifying medium in these experiments, has a diffusivity, $\kappa_{s}=1.5 \times 10^{-5} \mathrm{~cm}^{2} \mathrm{~s}^{-1}$, two orders of magnitude less than that of heat, $\kappa_{\theta}=1.5 \times 10^{-3} \mathrm{~cm}^{2} \mathrm{~s}^{-1}$. A balance is sought in the spinup process beween the time required for the fluid to come into approximately solid body rotation with the container, and the time within which diffusive processes act to distort the stratification and introduce a background thermal wind.

An early set of experiments investigated spinup with $N=0.7 \mathrm{~s}^{-1}$ and $f=[0.3,0.7,1.4] \mathrm{s}^{-1}$ for a free surface and for a rigid lid. When crystals of potassium permanganate were dropped into the tank they dissolved and left vertical traces in the fluid. The horizontal displacement of these traces outlined the structure of the velocity field. The displacements were monitored over a six hour period after the tank was filled. The free surface experiments showed azimuthal anticyclonic residual velocities increasing linearly from zero at the bottom boundary to a maximum at the free surface. Within first four hours the flows decreased by $55 \%$, and the decay process continued, though more slowly, over the following two hours. With the addition of a rigid lid, the potassium permanganate traces showed velocities peaking at middepth and decreasing to zero at the top and bottom boundaries. Furthermore, the velocity maximums were cut in half. The mixing experiments, therefore, were all conducted with rigid lids. The presence of the lid also served to eliminate surface waves during the mixing.

In the open basin experiments, a plexiglass lid was placed over the interior circular portion of the tank prior to filling. It was supported by styrofoam floats and rose with the incoming fluid. Four to six hours after the tank was filled and prior to the initiation of mixing, the background velocity field was checked through observation of potassium permanganate traces. The magnitude of the velocities was $O\left(10^{-2}\right) \mathrm{cm} / \mathrm{s}$, and the vertical structure and horizontal direction of the flow showed no distinct pattern. The residual flows appeared negligible with respect to the mixing-induced circulations.

In the channel mixing experiments the rigid lid rested on top of the channel walls. The tank was filled until the lid was submerged, but during the fill period the lid was not 
in contact with the stratified fluid. Therefore the tank was allowed to spinup for a longer period of time, generally 8 to 11 hours overnight. Again the fluid was checked prior to mixing for the presence of relative flows. In the basin outside the channel the fluid was in approximately solid body rotation with no sign of flows greater than $10^{-3} \mathrm{~cm} / \mathrm{s}$. Within the channel anticyclonic eddying motions of $O\left(10^{-2}\right) \mathrm{cm} / \mathrm{s}$ were detected in a 3-4 $\mathrm{cm}$ thick boundary layer just beneath the rigid lid. Otherwise, the fluid was quiescent.

For the channel experiments, as well as the open basin experiments, observed background velocities appeared negligible compared to the displacements caused by the mixing itself.

\subsection{Properties of bar-generated turbulence}

Turbulent mixing was produced by a vertically oscillating horizontal bar. Figure 3.2 provides a side view of the bar and plate. The bar had a fixed crosssection $1.27 \times 1.27 \mathrm{~cm}^{2}$ and a variable length, $15 \leq L_{B} \leq 30 \mathrm{~cm}$. The mechanism had a sinusoidal motion with an amplitude, $A=0.7 \mathrm{~cm}$, and a frequency, $\omega=[7,14,28] \mathrm{s}^{-1}$. The bar produced a layer of mixed fluid, exported into the interior of the tank. The support plate did not produce any additional mixing except at its lower edge. This disturbance was reduced by chamfering the bottom of the plate and by adding a thin cover which left only the bar in contact with the surrounding fluid.

It is assumed that the scale of motions in the tank can be related to the properties of turbulent mixing by the bar. The Hopfinger and Toly (1976) parameterization of gridinduced turbulent velocities (2.4) and length scales (2.3) provides a possible framework for analyzing the experiments that are described in Chapters 4 and 5. Versions of the Hopfinger-Toly model were used in the analyses of Thorpe (1982), Browand and Hopfinger (1985), Maxworthy and Monismith (1988), and De Silva and Fernando (1998). These four grids and the original Hopfinger-Toly grid all involved wake-wake 
interactions of flow through a mesh. Parameterization of turbulent velocity took the form given in (2.4).

$$
u_{t}=C \frac{A^{\alpha} M^{2-\alpha} \omega}{d}
$$

where $A$ and $\omega$ are the amplitude and frequency of oscillation, $M$ is the mesh dimension, $d$ is the distance from the grid and $C$ and $\alpha \leq 2$ are empirically determined constants. Thorpe also applied the Hopfinger-Toly parameterization to the multibar solid plate grid of Ivey and Corcos (1982), and Ivey (1987) adapted it to the cyclindrical version of the grid as well. In these cases the bar spacing rather than the mesh opening was considered the key geometric feature of the grid.

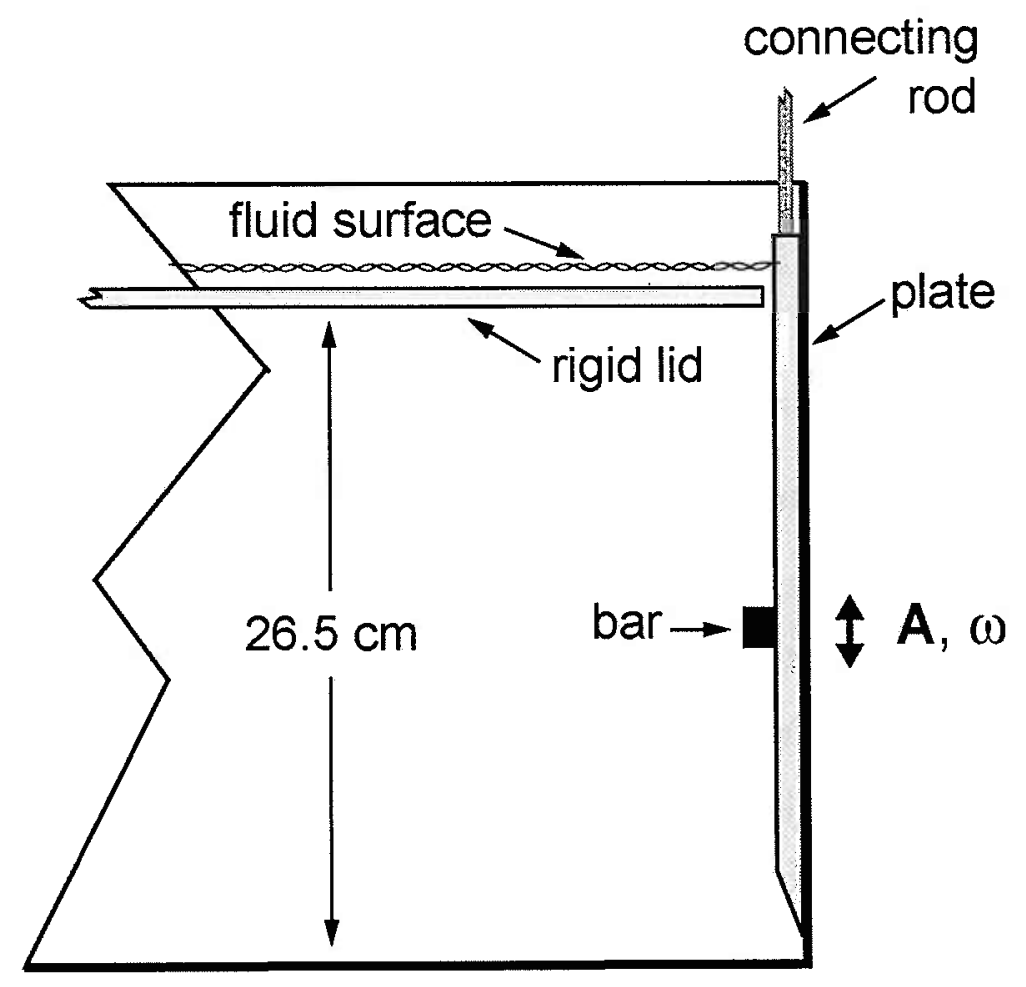

Figure 3.2: $\quad$ Side view of the mixing mechanism. The $1.27 \times 1.27 \mathrm{~cm}$ square horizontal bar is mounted at midddepth on a support plate, and oscillated vertically with amplitude $A$ and frequency $\omega$.

The mechanism used in the present experiments is a single bar mounted on a solid plate. The applicability of the Hopfinger-Toly model to the action of a single bar is 
investigated in a series of nonrotating experiments carried out in a small tank. The 30.5 $\mathrm{cm}$ long bar and plate assembly was mounted at one end of a 40.5 by $60 \mathrm{~cm}$ rectangular tank. The tank was filled with linearly stratified salt water to a depth of $26 \mathrm{~cm}$ and covered with a rigid styrofoam lid. Stratification was calculated from water samples taken from fixed locations. Five experiments were run with $N=[0.51,0.54,0.66,0.34,0.62] \mathrm{s}^{-1}$. The first two only ran for 15 minutes, long enough to observe the mixed layer develop and advance to the end of the tank. The last three experiments were run for an hour in order to observe the evolution of the turbulent zone after the mixed layer had covered a horizontal plane within the tank. The turbulent structures and mixed layer were visualized with shadowgraphs. All the experiments were photographed from the side and recorded by videotaping and computerized framegrabbing. The sequence of digital images was used for measuring turbulent properties as a function of time.

The shadowgraphs show a clearly defined turbulent zone forming above and below the bar, the emergence of an intrusion of mixed but no longer turbulent fluid, and the subsequent development of both the turbulent zone and the mixed layer. These features are labelled in Figure 3.4. While the main body of experiments is designed to examine the distribution of mixed fluid before it is blocked by the finite dimension of the laboratory tank, the small tank experiments have allowed observation of some longer term characteristics of the mixing.

In Figure 3.3 images from the experiment with $N=0.66 \mathrm{~s}^{-1}$ show both the evolution of the turbulent region and the outflow of mixed fluid. Turbulent overturning is already apparent at time $t=5 \mathrm{~s}$. Panel $a$ shows small scale structures in front of the bar and distinct eddies of mixed fluid above and below the bar. Panel $b$ shows the intruding layer of nonturbulent mixed fluid at $t=75 \mathrm{~s}$, and panel $c$ shows the layer at $t=730 \mathrm{~s}$, after mixed fluid has crossed the tank and returned to the bar. As seen in panel $d$ at $t=3105 \mathrm{~s}$, the layer thickens uniformly over time and the density interface between the mixed fluid and the ambient stratification sharpens. 

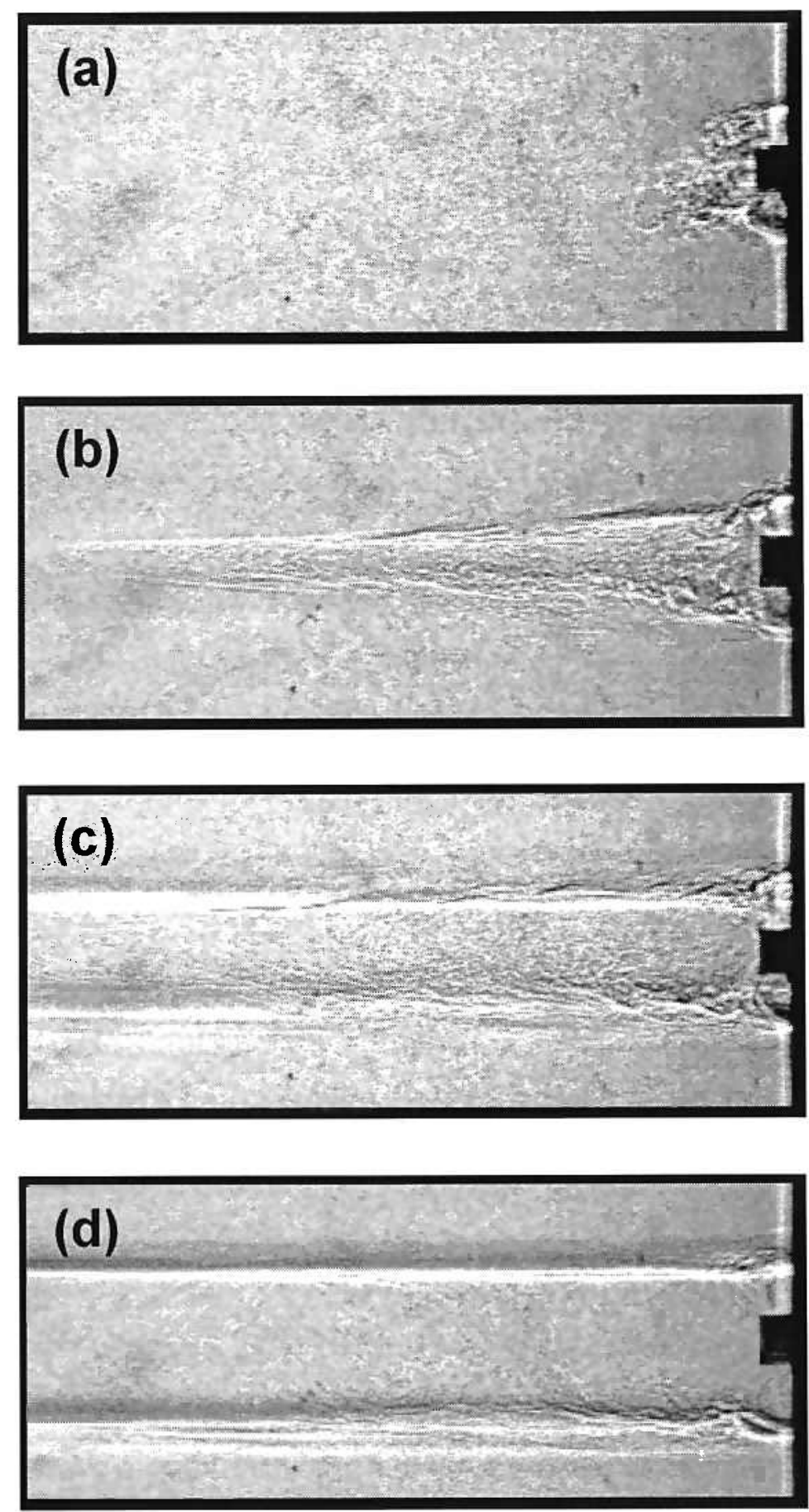

Figure 3.3: Side view shadowgraphs of the turbulent zone and mixed layer in an experiment with $N=0.66 \mathrm{~s}^{-1}, A=0.7 \mathrm{~cm}$, and $\omega=14 \mathrm{~s}^{-1}$. (a) Turbulent overturning at time, $\mathrm{t}=5 \mathrm{~s}$; (b) Emergence of mixed layer at $\mathrm{t}=75 \mathrm{~s}$; (c) Mixed layer at $\mathrm{t}=730 \mathrm{~s}$; (d) Mixed layer at $\mathrm{t}=3105 \mathrm{~s}$. 
In the experiment with $N=0.62 \mathrm{~s}^{-1}$, dye injected in front of the bar served as an additional marker of the mixed fluid flow. The dye showed mixed fluid moving from the face of the bar straight across the tank to the far wall, returning along both sides of the tank, then reentering the turbulent zone at the same horizontal level. The combined shadowgraph and dye image in Figure 3.4 shows the superposition of the mixed layer and the return flow at $t=660 \mathrm{~s}$.

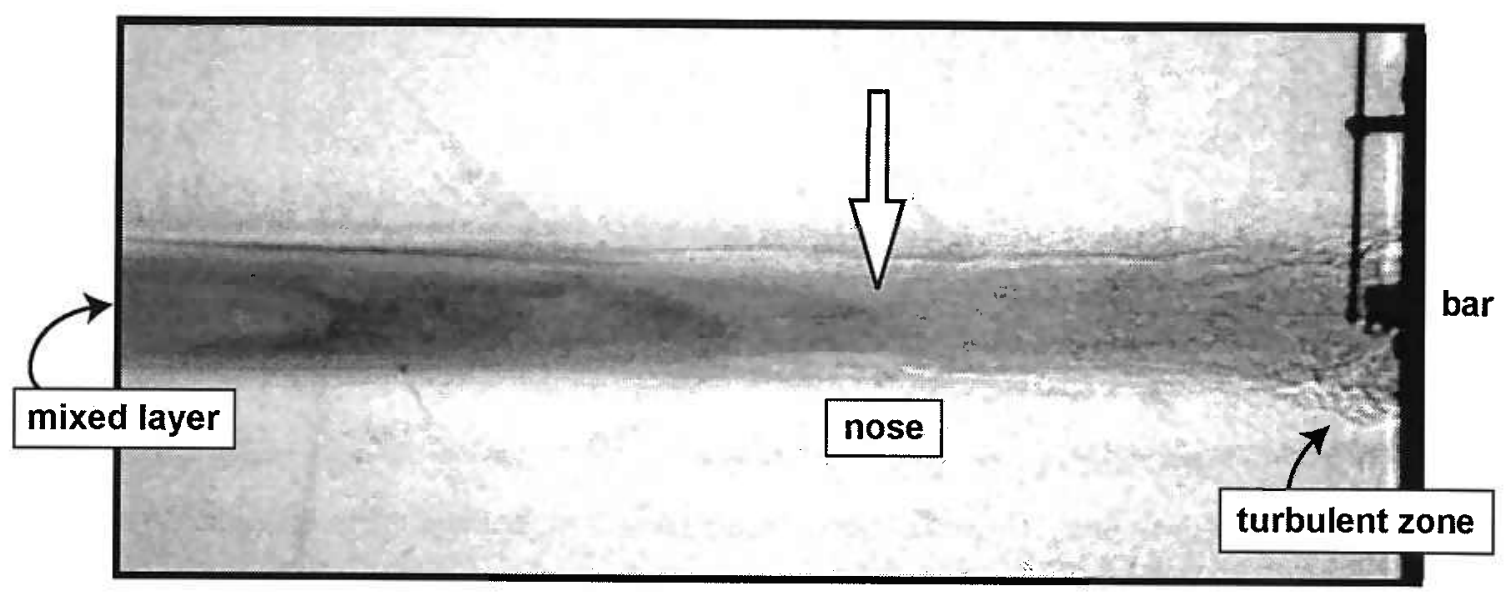

Figure 3.4: Side view of combined shadowgraph and dye image for an experiment with $N=0.62 \mathrm{~s}^{-1}$. The dye shows the superposition of the outflow from the bar and a return flow along the sides of the tank at the same depth at $\mathrm{t}=660 \mathrm{~s}$. The arrow points to the nose of the return flow. The turbulent zone is measured at the bar. The mixed layer height is measured at the left side of the image, $38 \mathrm{~cm}$ from the bar.

Figure $3.5 a$ presents data from the $N=0.66 \mathrm{~s}^{-1}$ experiment, plotting the height versus time for the turbulent zone and the mixed layer. The turbulent zone, measured at the bar, exhibits a rapid initial growth. Within the first 60 seconds it grows to the height that is maintained during the emergence of the mixed layer. The mixed layer is measured at a distance $38 \mathrm{~cm}$ from the bar. The steep increase in layer height shown between $t=200$ and $600 \mathrm{~s}$ indicates the arrival of the tapered intrusion, Figure $3.3 \mathrm{~b}$, at the measurement site. Once the intrusion has been blocked by the far wall, the mixed layer begins to thicken uniformly, Figure $3.3 c$ and $d$, and the turbulent zone resumes growing. Mixed layer growth goes as $h \sim t^{1 / 5}$ while the turbulent zone increases at a lesser rate 
with $h \sim t^{1 / 9}$. As the mixed layer height approaches the height of the turbulent zone, the data suggest that the turbulent zone may cease growing.

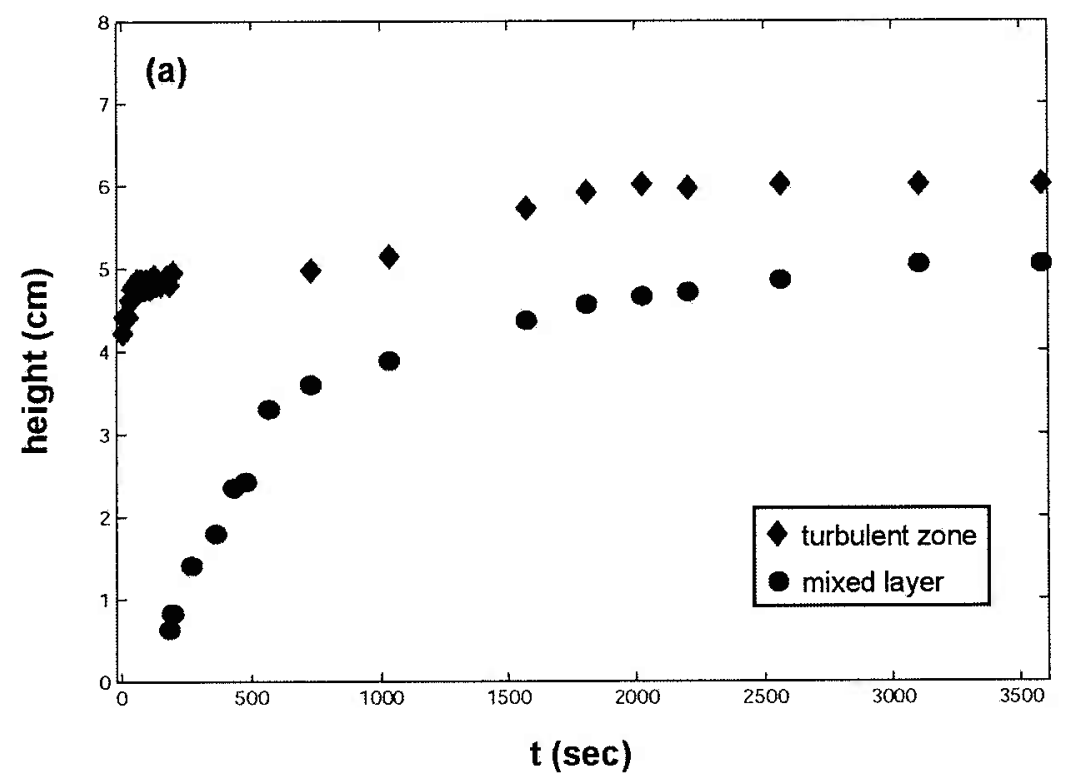

Figure 3.5 (a): Height vs time. Growth of the turbulent layer at the bar and the mixed layer $28 \mathrm{~cm}$ away from the bar for an experiment with $N=0.66 \mathrm{~s}^{-1}$.

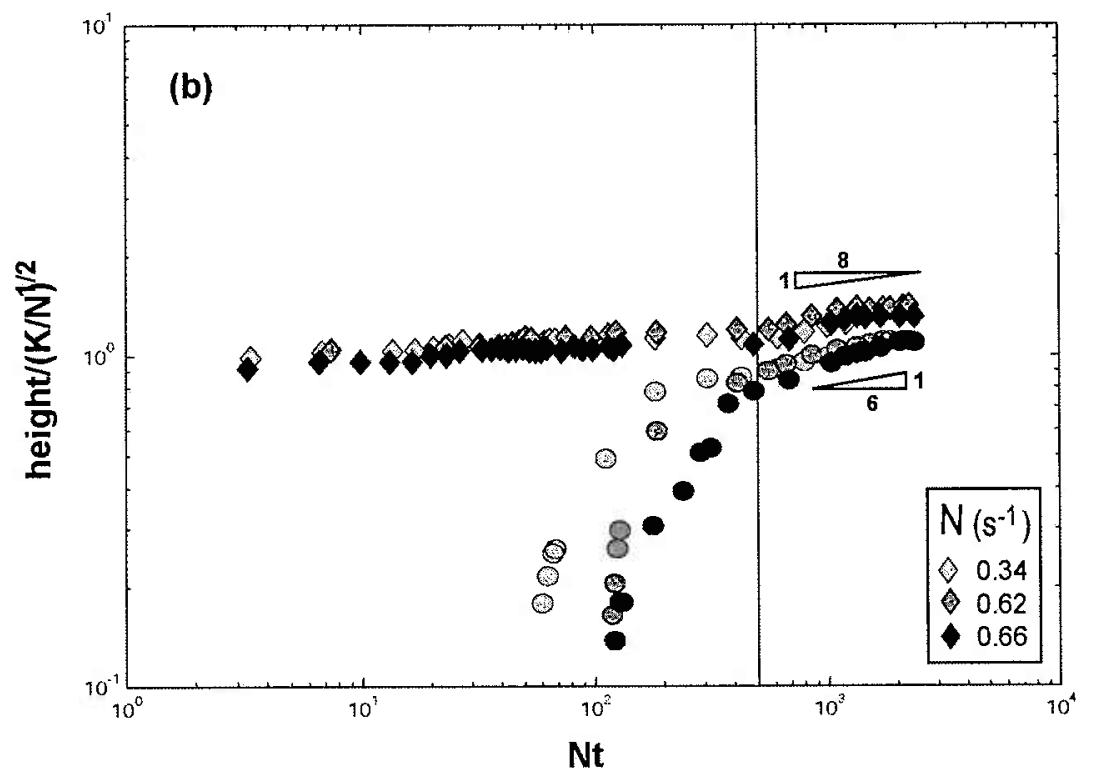

Figure3.5 (b): Height normalized by $(\mathrm{K} / \mathrm{N})^{1 / 2}$ vs Nt. Growth of the turbulent zone and mixed layer for experiments with $N=0.34,0.62$ and $0.66 \mathrm{~s}^{-1}$. 
Figure $3.5 b$ is a $\log \log$ plot of data from the three long experiments with time normalized by $N^{-1}$ and height normalized by $(K / N)^{1 / 2}$, where $K$ is the grid action (2.5) and is assigned an arbitrary value, $K=14 \mathrm{~cm}^{2} \mathrm{~s}^{-1}$, based on the bar frequency, $\omega=14 \mathrm{~s}^{-1}$. The combined data show the turbulent zone growing approximately as $t^{1 / 8}$ and the mixed layer growing approximately as $t^{1 / 6}$ for $N t \geq 500$. The longterm growth rate of the turbulent zone is not consistent with the observations of DeSilva and Fernando (1998) which show both the turbulent patch and the mixed layer growing as $t^{1 / 5}$. The experiments show a horizontal recirculation, Figure 3.4, but no visual evidence of horizontal entrainment of unmixed fluid into the turbulent zone at the top and bottom edges of the intrusion. The limited results more closely correspond to the entrainment hypotheses of Turner (1973) and Long (1978a) which postulate longterm growth due to vertical entrainment at $t^{1 / 8}$ and $t^{1 / 9}$ respectively.

In general, the experiments show that the oscillating bar generates turbulent overturning that is confined to the immediate vicinity of the bar by the ambient stratification. The mixing produces a single intrusion of mixed fluid similar to the intrusions generated by the horizontally oriented planar grids of Maxworthy and Monismith and DeSilva and Fernandes. However, the single bar mechanism is a unique design and it is still necessary to examine the relationship between its properties and the properties of the induced turbulence. The examination will follow the approach taken by Thorpe (1982) in inferring turbulent velocities and length scales from observations of the collapse of the turbulence.

Thorpe's method, summarized below, is discussed in Chapter 2.3.d. Thorpe used a vertically oriented planar grid in a linearly stratified fluid. The grid produced localized turbulence which collapsed into multiple layers of mixed fluid. At the time when the layers first appeared, Thorpe measured two characteristics of the flow: the position of the turbulent front and the height of the intrusion, $x_{F}$ and $h_{i}$, respectively, as indicated in Figure 2.4. He assumed that the frontal position represented $d_{N}$, the critical distance from the grid where buoyancy suppresses turbulent overturning, and that the layer heights 
approximated $l_{t}$, the length scale of the turbulence. He constructed expressions for both $d_{N}(2.26)$ and $l_{t}$ at $d_{N}(2.27)$ by using the Hopfinger and Toly grid parameterization of turbulent velocity $u_{t}$ (2.4) and length scales $l_{t}(2.3)$ with the respective constants of proportionality, $C$ and $\mathcal{B}$, left undetermined. He then put the observed measurements of position and height into (2.26) and (2.27) and solved for $C$ and $\mathcal{B}$. Thorpe applied the same methodology to the Ivey and Corcos (1982) experiment and computed different values for the constants, $C$ and $\mathcal{B}$, associated with the solid-plate multibar grid.

In the present single-bar experiments, neither bar size nor amplitude are varied, so the parameterization for turbulent velocity is written solely in terms of grid frequency $\omega$ and distance from the bar $d$,

$$
u_{t}=G \frac{\omega}{d},
$$

where $G$ replaces $C A^{\alpha} M^{2-\alpha}$ and has the dimensions of length squared. The parameterization of turbulent length scale is given as before (2.3),

$$
l_{t}=\mathcal{B} d \text {. }
$$

In order to evaluate the turbulent velocity (3.2) the constant of proportionality $\mathcal{B}$ has to be specified. It is assumed that the turbulent length scales for the single bar mechanism are similar to those of the multibar Ivey and Corcos grid. Thorpe's computed value for the planar grid, $\mathcal{B}=0.24$, is close to the value Ivey estimated for the cylindrical version, $\mathcal{B}=0.19 \pm 0.04$. The value $\mathcal{B}=0.24$ will be used for the single bar.

With (3.2) and (3.3) Thorpe's expression for the critical distance, $d_{N}$, becomes

$$
d_{N}=\frac{G^{1 / 2}}{\mathcal{E}^{1 / 2} \mathcal{B}^{1 / 2}}\left(\frac{\omega}{N}\right)^{1 / 2}
$$

Thorpe adopted the constant $\mathcal{E}(2.25)$ from the Dickey and Mellor (1980) finding that buoyant suppression of three dimensional turbulence occurs at a fixed value of the turbulent Froude number. That value is given by $\mathcal{E}=0.27$. The last constant, $\mathcal{G}$, is to be empirically determined for the single bar mechanism. 
$G$ is evaluated by measurements from the experiment with $N=0.66 \mathrm{~s}^{-1}$. In the Thorpe and Ivey and Corcos experiments, the time and location for data collection were established by the emergence of weakly turbulent mixed layers from a visible turbulent front. A similar procedure is not possible in experiments with only a single intrusion. Here, the timing is determined by the observed vertical growth of the turbulent zone. The capping of the initially rapid growth is considered coincident with the buoyant suppression of turbulent overturning. It occurs at a time $70 \leq t \leq 85 \mathrm{~s}$ or, in nondimensional terms, $45 \leq N t \leq 55$. The critical distance $d_{N}$ is identified with the location where the ratio of mixed layer height to distance from the bar is equal to the constant $\mathcal{B}$. The mean value of the location during the relevant time period was used in the formula for $d_{N}$ (3.4) and the equation was solved for $G$. The resulting value, $\mathcal{G}=0.23 \mathrm{~cm}^{2}$, gives a parameterization for turbulent velocity,

$$
u_{t}=0.23 \frac{\omega}{d},
$$

which, together with the turbulent length scale,

$$
l_{t}=0.24 d,
$$

gives formulae for the critical distance (3.4) and the turbulent length scale (3.3) evaluated at the critical distance.

$$
\begin{aligned}
d_{N} & =1.9\left(\frac{\omega}{N}\right)^{1 / 2}, \\
\left.l_{t}\right|_{d_{N}} & =0.45\left(\frac{\omega}{N}\right)^{1 / 2} .
\end{aligned}
$$

The predictions were applied to the shadowgraph experiments with $N=[0.51,0.54,0.34,0.62] \mathrm{s}^{-1}$. The heights of the mixed layer were measured at the predicted critical distances during the periods, $45 \leq N t \leq 55$. The observations matched the predicted values to within $20 \%$. The greatest mismatch occurred in the experiment with the dyed mixed layer. Otherwise the shadowgraph image data were within 2 to $9 \%$ 
of the predicted values. Figure 3.6 plots the mean heights normalized by the predicted heights (3.8). The errorbars show the standard deviations from the mean values.

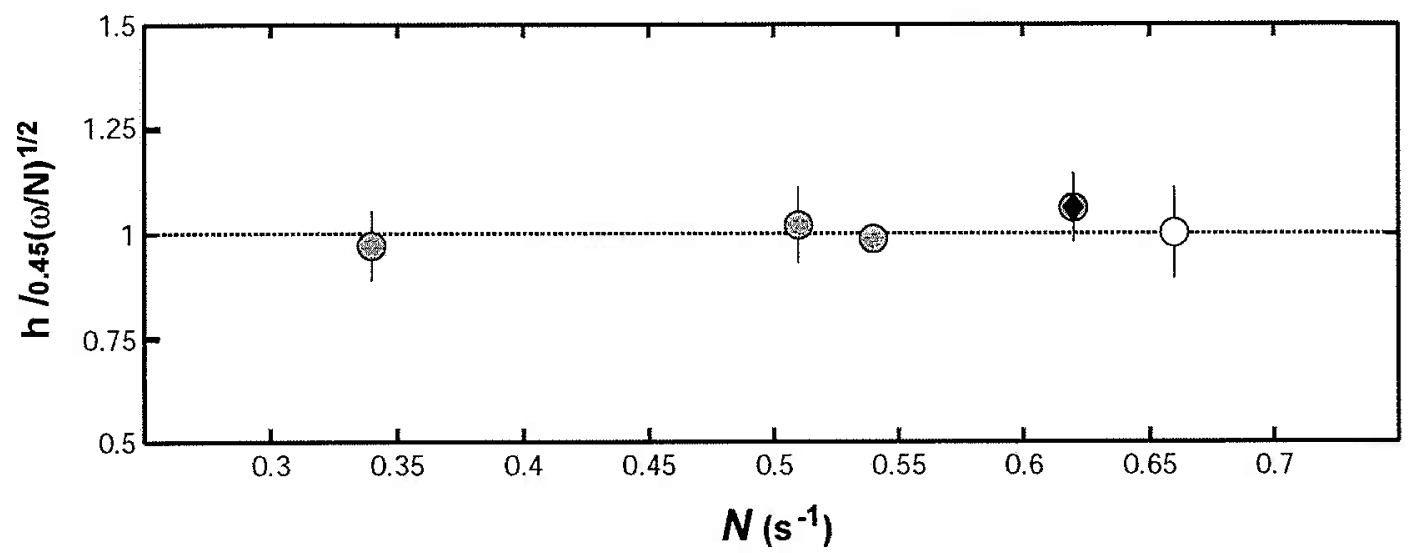

Figure3.6: Mean heights between $45 \leq N t \leq 55$, measured at the critical distance (3.7) and normalized by the predicted height (3.8). Error bars show the standard deviation from the mean. The white circle indicates the base case from which (3.7) and (3.8) are derived. The circle with the diamond indicates the experiment with the dyed mixed layer.

The above examination is not an independent test of the applicability of the Hopfinger-Toly model of grid-induced turbulence. Turbulent velocities and length scales are not directly measured in these experiments. However, the inferred values for the constants, $\mathcal{B}$ and $\mathcal{G}$, yield plausible and consistent predictions for the mixed layer. In Section 3.5 the parameterizations of bar-generated turbulence (3.5) and (3.6) will be incorporated into the definition of length scales used in analyzing the main body of rotating stratified experiments.

\subsection{Measurement methods}

In the open basin and channel experiments, the effects of the oscillating bar on the ambient fluid were monitored by dying the mixed fluid and by using a conductivity probe to measure density. The main visual record relies on dye injected directly in front of the 
bar. The dye mixed quickly throughout the turbulent region and marked the subsequent distribution of mixed fluid. The overhead video camera photographed both a top view and a reflected side view of the spreading dye. These images were recorded on video tape and in precisely timed computer sequences. The digitized computer frames provide the vertical and horizontal measurements of the dyed layer as a function of time.

The initial stratification of each experiment was measured using a Precision Measurement Engineering conductivity probe, which is calibrated for each experiment. In addition, the probe tracked the temporal evolution of the density field. Vertical profiles of conductivity measurements were made from approximately $5 \mathrm{~cm}$ beneath the rigid lid to within $2 \mathrm{~cm}$ of the sand bottom. In the open basin experiments the probe was used at two locations, 18.7 and $93.3 \mathrm{~cm}$ in front of the bar. The density profiles registered the arrival of mixed fluid at these sites and the development of the mixed layer. This data is analyzed in Chapter 4 and used to evaluate the effectiveness of the dye as a marker. In the channel experiments the probe was used to make repeated density sections across the channel. This data documents horizontal variations in the height of the mixed layer, and the horizontal density differences are used to infer transport along the channel.

\subsection{Experimental Parameters}

The experiments are characterized by nondimensional parameters that describe the property of the working fluid, the turbulent forcing, and the geometric configuration. The primary independent variables are the buoyancy frequency $N$, and Coriolis frequency $f$. In the experiments with vertical boundary mixing in an open basin, $N=[0,0.21,0.35,0.70] \mathrm{s}^{-1}$ and $f=[0,0.35,0.70,1.40] \mathrm{s}^{-1}$, giving $\frac{1}{4} \leq N / f \leq 2$. The range of $N / f$ is extended in the channel experiments where $N=[0.3,0.6,0.9] \mathrm{s}^{-1}$ and $f=[0,0.1,0.2,0.3,0.6,0.9,1.2] \mathrm{s}^{-1}$ giving $\frac{1}{4} \leq N / f \leq 9$.

Two other important independent variables are bar length and oscillation frequency. The frequency $\omega$ is a factor in the induced turbulence and its function is examined in the 
open basin experiments with $\omega=[7,14,28] \mathrm{s}^{-1}$. In all cases the bar frequency is much higher than the buoyancy and Coriolis frequencies, $\frac{\omega}{N}, \frac{\omega}{f} \gg 1$, and the bar motion creates localized turbulent mixing rather than internal or inertial waves.

The bar length $L_{B}$ or channel width $L_{C}$ determines the horizontal size of the mixing region. Its role is explored in both sets of experiments. In the open basin experiments the bar length, $L_{B}=[15.2,20.3,22.9,30.5] \mathrm{cm}$, is much smaller than the tank diameter $L_{T}$ : $L_{B} / L_{T} \ll<1$, where $L_{T}=112 \mathrm{~cm}$. The bar length and its location at middepth, away from the top and bottom boundaries of the tank, leave the mixing region exposed to both lateral and vertical exchanges with the ambient fluid. In the channel experiments the channel width $L_{C}$ is identical with the bar length and $L_{C}=[20.3,25.4,30.5] \mathrm{cm}$.

The main results of the mixing experiments are recorded in measurements of height, area and volume as functions of time. Scaling laws are used to connect the observations with external parameters and to identify the underlying physical processes.

The length scales used in evaluating the experimental results include the length of the mixing region, given by $L_{B}$ or $L_{C}$, and three scales, described below, that are constructed from the independent variables, $N, f$ and $\omega$; the buoyancy, Coriolis and bar frequencies.

Two scales follow from the assumption that scales of motion at the interface of the turbulent zone and the ambient rotating stratified fluid are set by a balance between the intrinsic time scale of the turbulence, $l_{t} / u_{t}$, and the buoyancy and rotation periods, $N^{-1}$ and $f^{-1}$. The Chapter 2 review of laboratory studies established that turbulence is suppressed by stratification when the turbulent Froude number (2.10),

$$
F r_{t} \equiv \frac{u_{t} / l_{t}}{N} \leq 1
$$

and by rotation when the turbulent Rossby number (2.32),

$$
R o_{t} \equiv \frac{u_{t} / l_{t}}{f} \leq 1 .
$$


When expressed in terms of the parameterizations of bar-generated turbulence, (3.2) and (3.3), these relations imply the distances from the bar for the onset of the buoyant and rotational inhibition, respectively,

$$
\begin{gathered}
d_{N} \propto\left(\frac{G}{\mathcal{B}}\right)^{1 / 2}\left(\frac{\omega}{N}\right)^{1 / 2} \text { and } \\
d_{f} \propto\left(\frac{G}{\mathcal{B}}\right)^{1 / 2}\left(\frac{\omega}{f}\right)^{1 / 2} .
\end{gathered}
$$

The factors, $\mathcal{G}=0.23 \mathrm{~cm}^{2}(3.5)$ and $\mathcal{B}=0.24$ (3.6), can be written as a single constant of proportionality,

$$
\gamma \equiv(G / \mathcal{B})^{1 / 2}=0.98 \approx 1(\mathrm{~cm})
$$

The constant $\gamma$ has the dimension of length and is $O(1) \mathrm{cm}$.

A buoyancy height scale, $H_{N}$, is defined from (3.11.a) and (3.12) for $F r_{t}=1$,

$$
H_{N} \equiv \gamma\left(\frac{\omega}{N}\right)^{1 / 2}(\mathrm{~cm})
$$

Similarly, a rotational length scale, $L_{f}$, is defined from (3.11.b) and (3.12) for $R o_{t}=1$,

$$
L_{f} \equiv \gamma\left(\frac{\omega}{f}\right)^{1 / 2}(\mathrm{~cm})
$$

The third length scale used in analyzing the experimental results is the internal Rossby radius of deformation, hereafter referred to as the deformation radius $L_{R}$.

$$
L_{R}=\frac{N}{f} H_{N}(\mathrm{~cm}),
$$

where $H_{N}(3.13)$ is the scale height of the mixed layer. Horizontal density gradients cause the gravitational collapse of mixed fluid at the edge of the turbulent zone and a laminar outflow into the interior. The deformation radius is the horizontal scale over which rotational effects on the outflow become as important as the buoyancy effects.

The laboratory experiments tested the applicability of the length scales, $H_{N}, L_{f}$ and $L_{R}$, to the observed flows over a wide range of values. In the open basin experiments, 
$4 \leq H_{N} \leq 9 \mathrm{~cm}, 3 \leq L_{f} \leq 7 \mathrm{~cm}$ and $1.6 \leq L_{R} \leq 12 \mathrm{~cm}$. In the channel experiments $4 \leq H_{N} \leq 7 \mathrm{~cm}, 3 \leq L_{f} \leq 12 \mathrm{~cm}$ and $1.8 \leq L_{R} \leq 35 \mathrm{~cm}$. The ratio of channel width to the deformation radius varies by more than an order of magnitude: $1 \leq\left(L_{C} / L_{R}\right) \leq 18$. 


\section{Chapter 4}

\section{Localized Mixing at a Vertical Boundary}

\subsection{Introduction}

The laboratory experiments described in this chapter explore the interaction of localized vertical boundary mixing with a body of linearly stratified, rotating fluid. The mixing mechanism, sketched in Figures 3.1 and 3.2, is a single horizontally oriented, vertically oscillating bar located at middepth along the outer wall of a rotating tank. The bar produces a small region of turbulence open to both vertical and horizontal exchanges with the ambient fluid.

The turbulent region quickly reaches a steady state height and collapses into the interior. Surprisingly, the export of fluid does not occur as a boundary current. Instead, the mixed layer moves directly out from the bar as a radial front of uniform height. The export dynamics are affected by an observed lateral entrainment flow toward the mixing zone. This flow pattern demonstrates a difference between generating mixed fluid locally and simply introducing it as a mass source. The quantitative analysis, which follows, suggests that the production and distribution of mixed fluid are tied to the interaction of turbulence with the ambient stratification and rotation. Models of lateral and vertical entrainment are discussed, and evaluated through experiments in which the mixing bar length is varied. Finally, rotating and nonrotating experiments are compared. 


\subsection{Qualitative description}

\section{2.a Export of mixed fluid from the turbulent zone}

Turbulent mixing of stratified fluid begins with the onset of the bar oscillation at $t=0$.

The typical development of the mixed layer is seen in images from an experiment with $(\omega, N, f)=(14,0.86,0.88) \mathrm{s}^{-1}$ (Figure 4.1). Within the first 30 seconds a turbulent dyed region has formed around the bar and mixed fluid has collapsed into an outwardly moving intrusion (panel $a$ ). At $t=60 \mathrm{~s}$ (panel $b$ ), the turbulent region at the bar has reached its full height and the emergent mixed layer is well-defined. Panels $c$ and $d$ show that the layer height remains uniform as mixed fluid advances into the interior $(t=300$ and $600 \mathrm{~s})$.
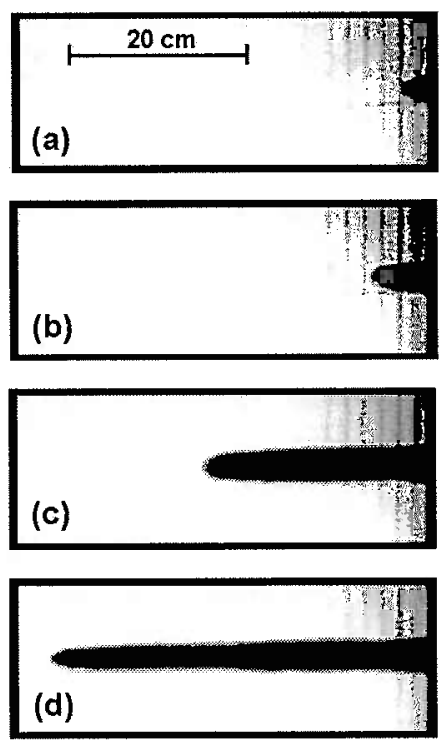

Figure 4.1: $\quad$ Dye images for an experiment with $(\omega, N, f)=(14,0.86,0.88) \mathrm{s}^{-1}$ and $L_{B}=20.3 \mathrm{~cm}$. Side view of mixed layer. (a) $\mathrm{t}=30 \mathrm{~s}$, (b) $\mathrm{t}=60 \mathrm{~s}$, (c) $\mathrm{t}=300 \mathrm{~s}$, (d) $\mathrm{t}=600 \mathrm{~s}$. 
Video images from experiments with $\frac{1}{3} \leq N / f \leq 3$ indicate that vertical mixing is restricted by stratification, while the lateral distribution of mixed fluid depends on both $N$ and $f$. Figure 4.2 shows mixed layers at $t=360 \mathrm{~s}$ from four different experiments. The experiments have identical grid frequencies, $\omega=14 \mathrm{~s}^{-1}$, but different values of the buoyancy and Coriolis frequencies. Compare panels $a$ and $b$ where $N=0.3 \mathrm{~s}^{-1}$, and $f=0.3$ and $0.9 \mathrm{~s}^{-1}$, respectively, with panels $c$ and $d$ where $N=0.9 \mathrm{~s}^{-1}$ and, again, $f=0.3$ and $0.9 \mathrm{~s}^{-1}$. The layer heights decrease with increasing stratification and are independent of the rotation rate. However, the length of the intrusion is affected by both fluid properties: greater length is associated with higher buoyancy frequencies and lower Coriolis frequencies.
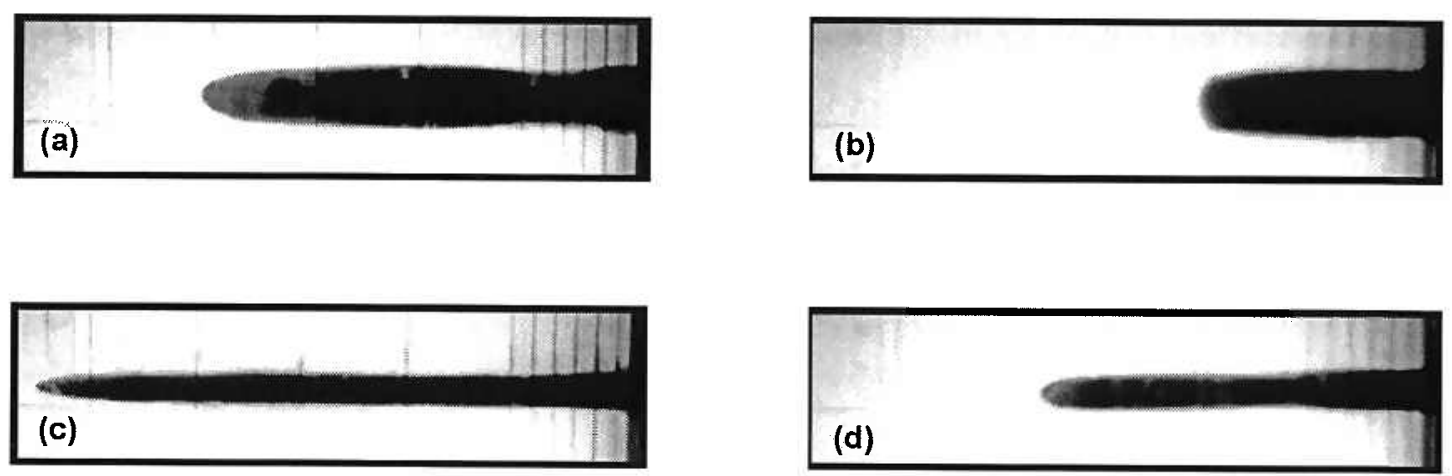

Figure 4.2: Side view dye images for four experiments with $\omega=14 \mathrm{~s}^{-1}$ and $L_{B}=20.3 \mathrm{~cm}$ at $\mathrm{t}=360 \mathrm{~s}$. (a) $N / f=1 ;(N, f)=(0.3,0.3) \mathrm{s}^{-1}$, (b) $N / f=1 / 3 ;(N, f)=(0.3,0.9) \mathrm{s}^{-1}$, (c) $N / f=3 ; \quad(N, f)=(0.9,0.3) \mathrm{s}^{-1}$, (d) $N / f=1$; $(N, f)=(0.9,0.9) \mathrm{s}^{-1}$. 
Figures 4.3 and 4.4 present top view images of the experiments with $N / f=3$ and $N / f=\frac{1}{3}$, respectively, and demonstrate the relationship between $N / f$ and the lateral spreading of the mixed layer. In both cases, the mixed fluid advances directly into the interior with no significant geostrophic boundary current formation. In Figure 4.3, where $N / f=3$, the emerging fluid moves directly across the tank (see panel $a$ and $b$ at $t=360 \mathrm{~s}$ and $t=900 \mathrm{~s}$, respectively). The dyed layer encounters the opposite wall at $t=1200 \mathrm{~s}$ and by $t=3600 \mathrm{~s}$ (panel $c$ ) it has spread over the entire interior. In Figure 4.4, where $N / f=\frac{1}{3}$, the front of mixed fluid never crosses the tank. At $t=360 \mathrm{~s}$ (panel $a$ ) the flow has gone less than one half the distance covered in the same time period by the flow in Figure $4.3 a$. By $t=900 \mathrm{~s}$ (panel $b$ ) a field of eddies has formed and the cross-tank progress of the coherent front has been arrested. Forty-five minutes later, at $t=3600 \mathrm{~s}$ (panel $c$ ), the eddies have grown and dispersed, but the mixed fluid is still confined near the bar.

All the vertical boundary mixing experiments show a similar pattern. Fluid, dyed at the bar, is exported directly out into the tank, with little or no fluid exported in a boundary current. The absence of dye at the sides of the mixing region suggests that entrainment of unmixed fluid may be occurring laterally at the level of the mixing bar. 

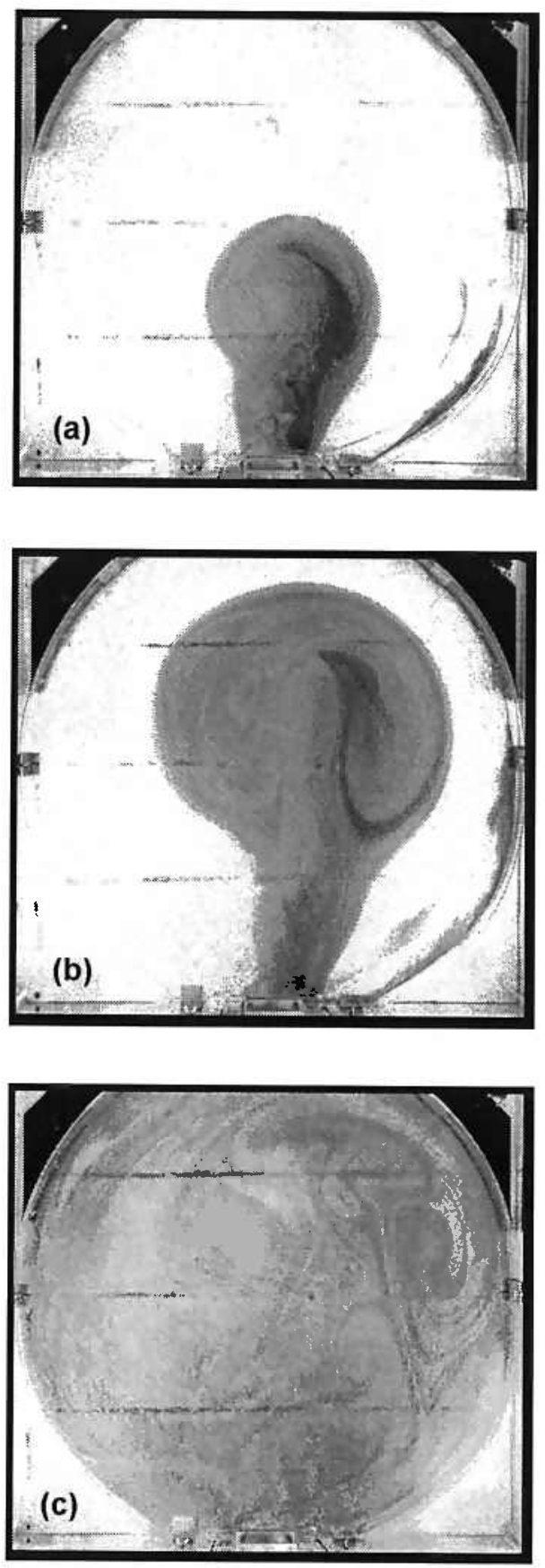

Figure 4.3: Dye images for experiment with $N / f=3 ;(\omega, N, f)=(14,0.9,0.3) \mathrm{s}^{-1}$ and $L_{B}=20.3 \mathrm{~cm}$. (a) $\mathrm{t}=360 \mathrm{~s}$, (b) $\mathrm{t}=900 \mathrm{~s}$, (c) $\mathrm{t}=3600 \mathrm{~s}$. 

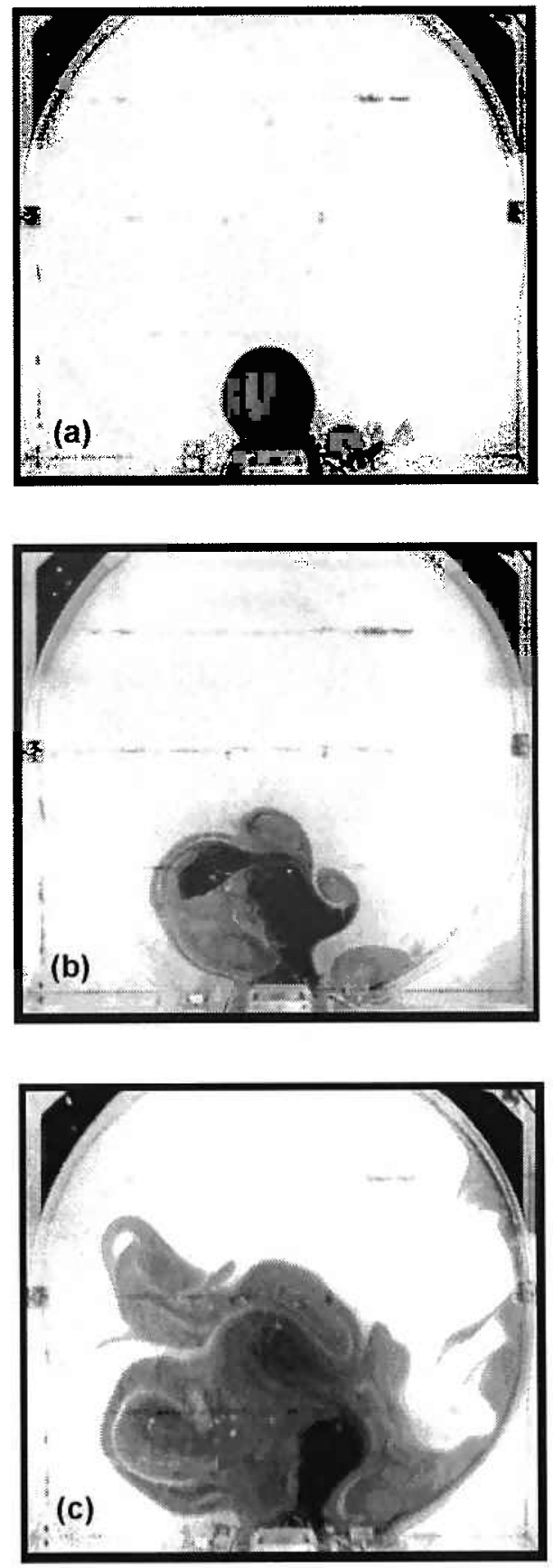

Figure 4.4: Dye images for experiment with $N / f=1 / 3 ;(\omega, N, f)=(14,0.3,0.9) \mathrm{s}^{-1}$ and $L_{B}=20.3 \mathrm{~cm}$. (a) $\mathrm{t}=360 \mathrm{~s}$, (b) $\mathrm{t}=900 \mathrm{~s}$, (c) $\mathrm{t}=3600 \mathrm{~s}$. 


\section{2.b Entrainment of unmixed fluid into the turbulent zone}

Circulation of ambient fluid outside the dyed layer is investigated through two flow visualization techniques. Potassium permanganate crystals are dropped at selected locations to create vertical dye streaks, and neutrally buoyant dye is laid down at specified depths to create horizontal dye streaks across the tank. The displacement of the dye columns traces the vertical structure of horizontal flows, while displacement of the horizontal dye lines provides information on flow across a section.

Cross-tank dye lines laid across the tank $24.5 \mathrm{~cm}$ in front of the bar are used to observe horizontal flows to and from the mixing region. Figure 4.5 diagrams a top view of a dye line experiment. The dotted line marks the original position of dye located at the approximate depth of the mixing bar. The solid line is representative of its position a short time later. The change in position indicates fluid displacement at the level of the mixed layer. Observations of horizontal cross-tank dye lines are made for experiments with values of $N / f=\left[\frac{1}{4}, \frac{1}{2}, 1,2\right]$.

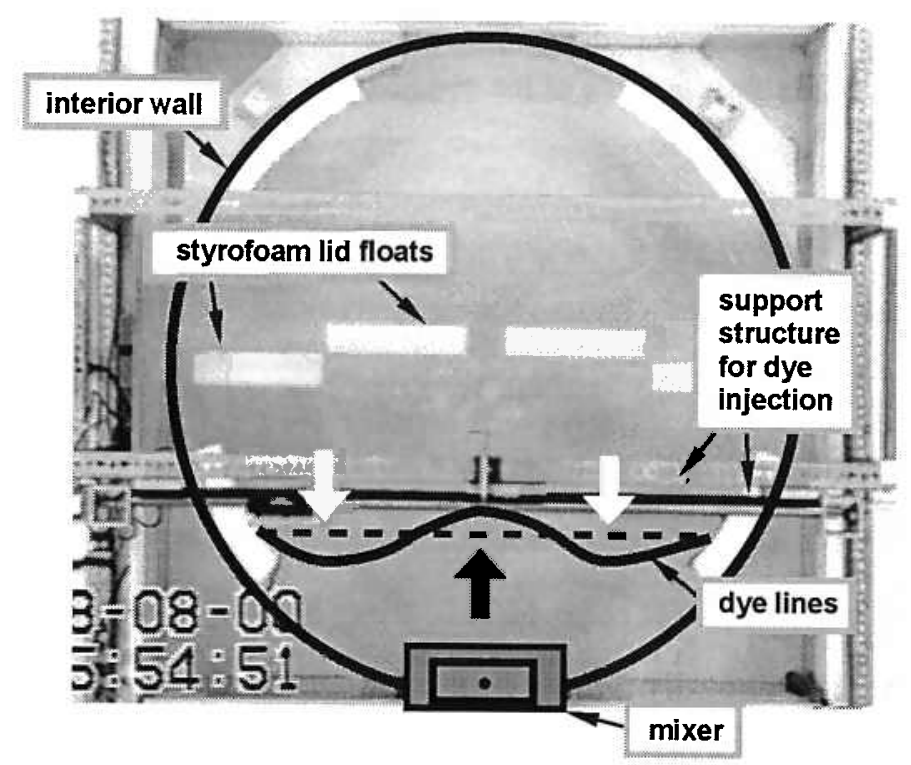

Figure 4.5: Top view of tank with sketch of dye lines. The dotted line marks the original position. The solid line indicates a typical displacement of the dye line. The dark horizontal line that crosses the tank above the dye lines is part of the dye injection system. The mixer is at the bottom of the image. 

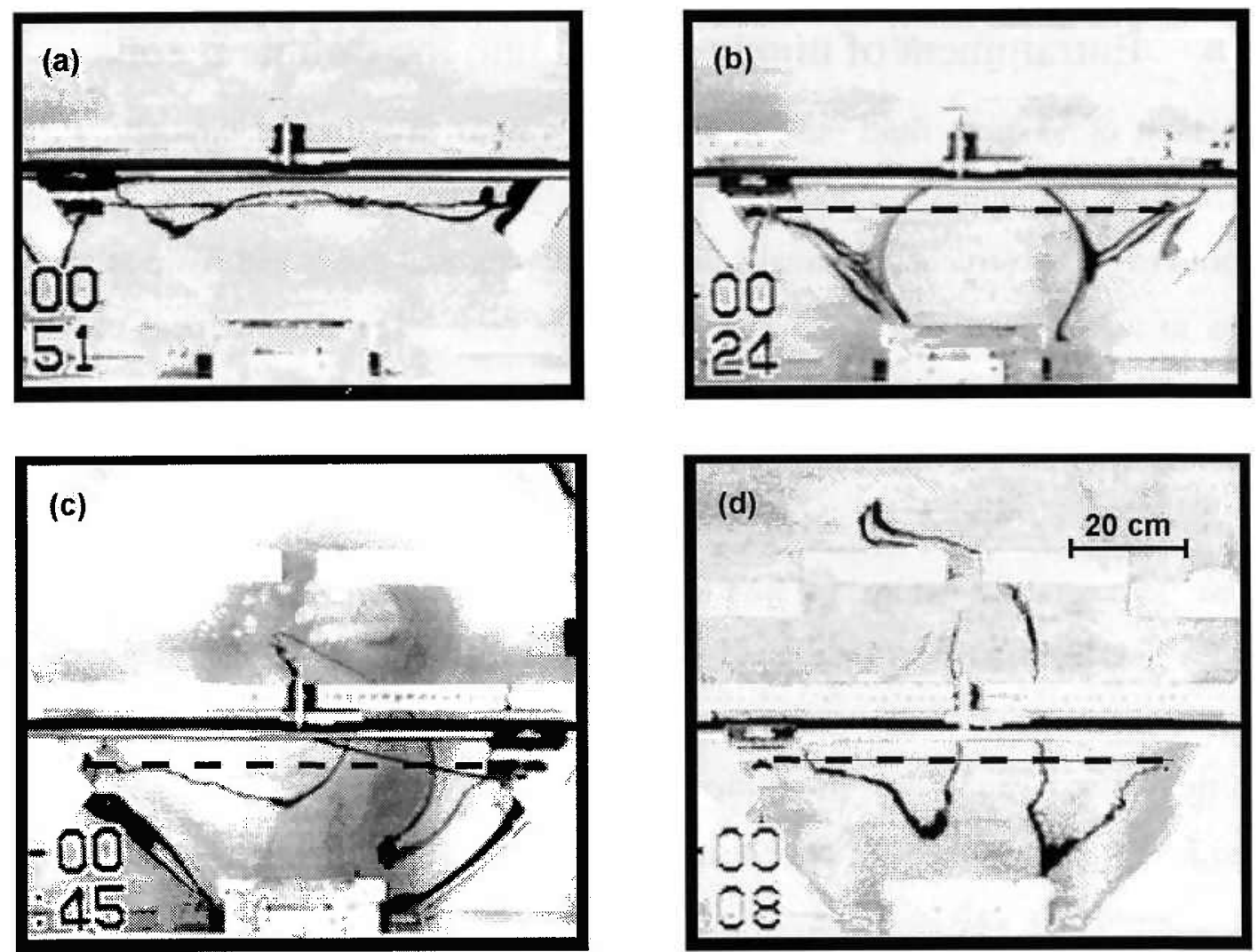

Figure 4.6: Dye line images for experiment with $N / f=1 ;(\omega, N, f)=(14,0.7,0.7) \mathrm{s}^{-1}$ and $L_{B}=30.5 \mathrm{~cm}$. The dotted lines indicate the original position of the dye lines. Images taken at times (a) $t=7 \mathrm{~min},(\mathrm{~b}) \mathrm{t}=10$ $\min ,(c) t=23 \mathrm{~min}$, (d) $\mathrm{t}=7 \mathrm{hr}$.

Figure 4.6, with examples from the experiment where $N / f=1$, typifies the observations. Panel $a$, at $t=7 \mathrm{~min}$, shows two dye lines: one, laid down at $t=5 \mathrm{~min}$, is still in place; the other, laid down at $t<0$, has been displaced outwards at the center and in toward the mixing bar at the sides. The outlined areas show a 5:3 ratio of inward to outward flow. The uneven flow ratio at this time may indicate that fluid is advected vertically as well as horizontally when mixing is initiated. Within three minutes, at $t=10 \mathrm{~min}$, both lines have been drawn into the the sides of the mixing region and a central outward flow is clearly established (panel $b$ ). Later dye line displacements show a mass balance at the level of the bar. The areas outlined in panel $c$ at $t=23 \mathrm{~min}$ have a 
1:1 ratio of inbound and outbound flow, and panel $d$ shows the 1:1 ratio still holding for a dye line laid down 7 hours into the experiment. The observations indicate that the export of mixed fluid and the inflow of ambient fluid balance each other at the level of the mixed layer. The dye line experiments imply that the production of mixed fluid relies on horizontal rather than vertical entrainment. Additional dye lines across the tank at five $\mathrm{cm}$ above and below the bar trace similar flows with much smaller velocities, suggesting that the circulation at the level of the mixed layer indicates the general pattern.

More information on the vertical structure of the horizontal velocity field comes from observations of the vertical dye traces left by potassium permanganate crystals. Dye columns are placed in advance of the intrusion of mixed fluid, intersecting the mixed layer, and in the undyed area close to the bar. The displacement of dye traces in advance of the intrusion shows little or no coherent motion, while the profiles through the mixed layer show a unidirectional velocity over the whole depth of the fluid column. The velocity reaches its maximum at the mixed layer level and drops off rapidly above and below it, in agreement with the pattern inferred from the horizontal dye lines. Profiles in undyed areas close to the grid indicate a horizontal flow of ambient fluid towards the mixing bar. Fluid at the level of the mixed layer is drawn into the sides of the turbulent zone along the top and bottom edges of the bar.

The velocity structure shown by the two flow visualization techniques indicates an entrainment process that is preferentially horizontal. The observations support a picture of a mixing region of finite vertical and horizontal dimension that draws stratified ambient fluid in along the walls of the tank at the level of the bar and exports mixed fluid away from the boundary directly into the interior at the same level.

\subsection{Quantitative analysis and scaling}

This section analyzes the measurable characteristics of the mixed outflow. The measurements cover the period from the emergence of the mixed layer up to the appearance of disturbances on the advancing front, and they include the height of the 
mixed layer, the advance of the intrusion into the interior, and the areal spreading and volume flux of mixed fluid. These characteristics are related to the independent variables, $N, f$ and $\omega$.

The initial fourteen experiments are conducted with a single bar size, $L_{B}=30.5 \mathrm{~cm}$. Eight experiments include both rotation and stratification with $0.33 \leq N \leq 0.75 \mathrm{~s}^{-1}$ and $0.35 \leq f \leq 1.40 \mathrm{~s}^{-1}$. One experiment each is run with a bar frequency $\omega=[7,28] \mathrm{s}^{-1}$. In all other cases, $\omega=14 \mathrm{~s}^{-1}$. In addition, three experiments have stratification, but no rotation, with $N=[0.21,0.35,0.69] \mathrm{s}^{-1}$, and three have rotation, but no stratification, with $f=[0.35,0.70,1.40] \mathrm{s}^{-1}$.

The measured quantities are fit to the independent variables through scaling laws that suggest the underlying dynamics. Section 4.4 offers a physical interpretation of the observations, and a model for the production of mixed fluid is tested through experiments using additional bar lengths, $L_{B}=[15.2,20.3,22.9] \mathrm{cm}$.

\section{3.a Data sources}

The main sources of quantitative data are the conductivity probe records and the images of the dyed fluid. The probe measurements provide a detailed look at the evolution of the vertical density structure. Figure 4.7 shows a time series of density vs depth profiles at one location for an experiment with $(\omega, N, f)=(14,0.68,1.40) \mathrm{s}^{-1}$. The first profile, at $t=300 \mathrm{~s}$, shows the initial linear density distribution. The dyed fluid has not yet reached the probe location $18.7 \mathrm{~cm}$ in front of the bar. At $t=900 \mathrm{~s}$ a step in the linear density profile marks the arrival of the intrusion fluid. By $t=1800 \mathrm{~s}$ the step height is well established. The superimposed dotted lines show five additional profiles taken between $t=2700 \mathrm{~s}$ and $t=14880 \mathrm{~s}$. The intrusion is characterized by a well mixed middle zone bounded by two zones of high density gradient. In the period after $t=1800 \mathrm{~s}$, the middle mixed zone grows from 2.8 to $3.9 \mathrm{~cm}$, while the overall vertical extent of the intrusion remains relatively constant. The intrusion height is defined as encompassing both the low 
gradient mixed middle and the high gradient boundaries. It is measured by the intersection points of the ambient, approximately linear, density curve with the tangents of the high gradient slopes. The probe data give an average intrusion height, $h_{p}=(4.5 \pm 0.2) \mathrm{cm}$. A second step, seen at the bottom of the density profiles in Figure 4.7, is due to mixing by the base of the grid support plate. In later experiments the edge of the plate has been chamfered and the secondary step is greatly reduced.

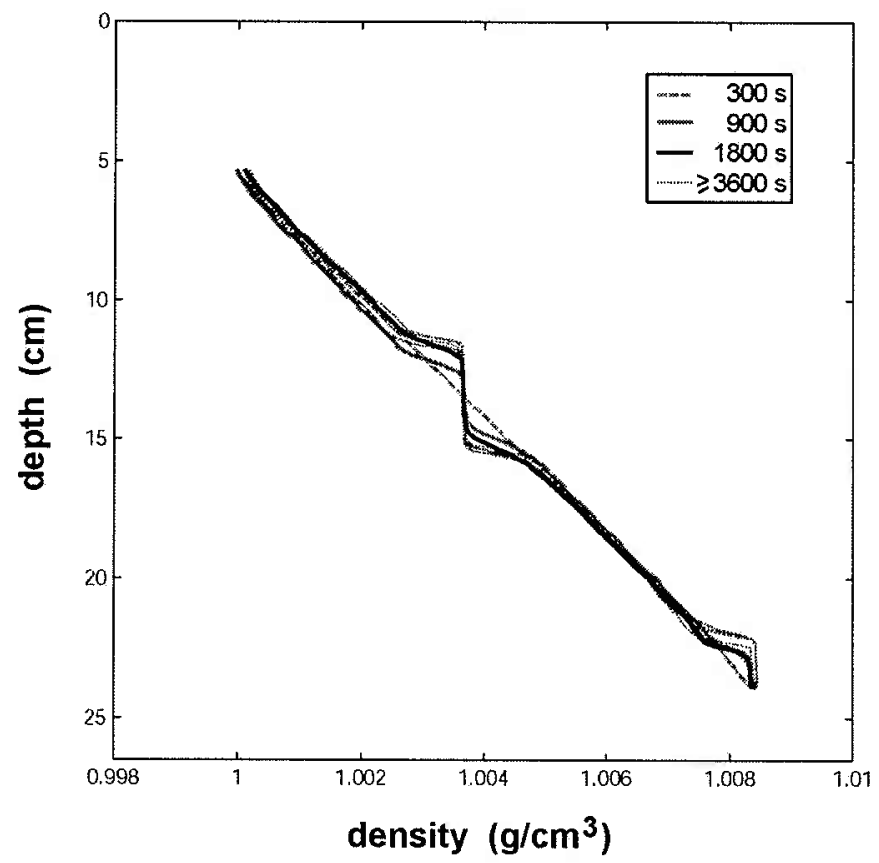

Figure 4.7: Density profiles for experiment with $(\omega, N, f)=(14,0.68,1.40) \mathrm{s}^{-1}$ and $L_{B}=30.5 \mathrm{~cm}$. Eight profiles taken $18.7 \mathrm{~cm}$ in front of the mixing bar between $\mathrm{t}=300 \mathrm{~s}$ and $\mathrm{t}=14880 \mathrm{~s}$.

The probe measurements at two locations, one $18.7 \mathrm{~cm}$ in front of the grid and the other $93.3 \mathrm{~cm}$ across the tank, register density changes only after the arrival of dyed fluid. The appearance of dye can therefore be associated with the presence of mixed fluid. A comparison of dyed layer thickness to probe profiled intrusion height indicates a consistent relationship between the two methods of measurements. 
The dye distributions seen in Figures 4.1 and 4.2 show one well-defined layer in the fluid. Within the first 100 seconds of bar motion, the dyed layer thickens and establishes a height that remains nearly steady over the timespan of the experiments and nearly uniform over the length of the intrusion. Height measurements, based on digitized video images, are taken near the bar where the images are sharpest. The site is marked by the doubleheaded arrow in Figure 3.1. Mean values for the dyed layer heights, $h_{d}$, in each experiment are calculated from data collected after the first 100 seconds. Corresponding mean values from the probe profiles, $h_{p}$, are calculated from data collected after the nose of the intrusion has passed the profiling site. The dyed layer in the experiment shown in Figure 4.7 has a mean height of $h_{d}=(4.6 \pm 0.2) \mathrm{cm}$ a value comparable to the $4.5 \mathrm{~cm}$ probe measurement.

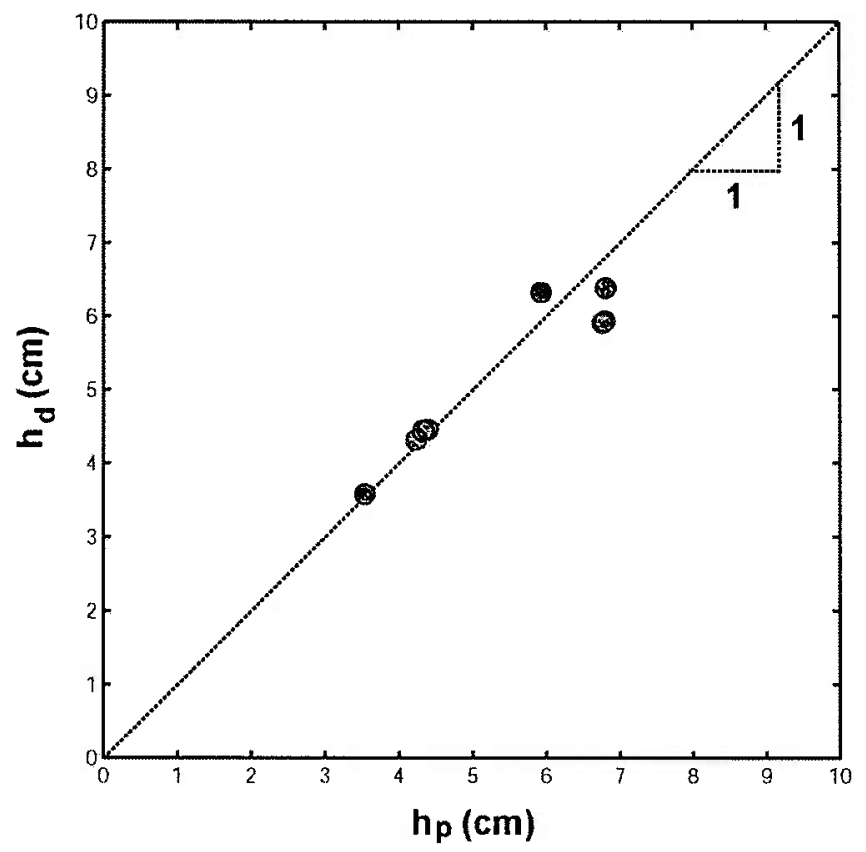

Figure 4.8: $\quad$ Mean dyed layer height, $h_{d}$, versus mean overall step height, $h_{p}$, in the density profile. Measurements for eight experiments with $7 \leq \omega \leq 28 \mathrm{~s}^{-1}, \quad 0.33 \leq N \leq 0.75 \mathrm{~s}^{-1}, \quad 0.35 \leq f \leq 1.40 \mathrm{~s}^{-1}$, and $L_{B}=30.5 \mathrm{~cm}$. 
Figure 4.8 compares layer heights taken from dye images, $h_{d}$, to heights determined from probe profiles, $h_{p}$. It demonstrates a close correspondence between the two sets of heights. For all the stratified, rotating experiments,

$$
h_{d}=(1.00 \pm 0.07) h_{p} .
$$

The dye distribution closely matches the vertical scale of the intrusion. Even though it does not measure the thoroughness of the mixing, it serves as a marker for flows of mixed fluid from the turbulent zone. In the following sections digitized video images of the dye are used to document and quantify the evolution of mixed fluid.

\section{3.b Vertical mixing and layer height}

Mean heights for the mixed layers in each experiment are based on the dye images and calculated as described in Section 4.3.a. The layer heights scale well with the buoyancy scale height, $H_{N}=\gamma\left(\frac{\omega}{N}\right)^{1 / 2}$ (3.13), which represents a balance between bar-generated turbulence and the ambient stratification. $\gamma$ is a constant of proportionality of $O(1) \mathrm{cm}$ (see Chapter3.3). Figure 4.9 plots mean mixed layer height, $h_{m}$, versus $\gamma\left(\frac{\omega}{N}\right)^{1 / 2}$. The errorbar on selected data points is the standard deviation about the mean height for the individual experiment. On average, the standard deviation is $5 \%$ of the mean value. A linear fit to the data gives,

$$
h_{m}=(0.97 \pm 0.05) \gamma\left(\frac{\omega}{N}\right)^{1 / 2} \mathrm{~cm} .
$$

The demonstrated dependence of layer height on $\gamma\left(\frac{\omega}{N}\right)^{1 / 2}$ is consistent with previously cited (see Chapter 2) nonrotating studies which established empirical and theoretical relationships between the height of intrusive layers and the relative strengths of ambient stratification and imposed grid mixing (Ivey and Corcos, 1982; Thorpe, 1982; Browand and Hopfinger, 1985). 
The mixed layer heights plotted in Figure 4.9 show no dependence on variations in the Coriolis frequency. The data are from experiments both with and without rotation, with $f=[0,0.35,0.70,1.40] \mathrm{s}^{-1}$. The rotating experiments of Davies et al. (1991) showed dependence of layer height on rotation after mixed fluid had circled the tank and returned to the source region. However, in their experiments the short term establishment of the mixed layer height was unaffected by rotation and also scaled with $\left(\frac{\omega}{N}\right)^{1 / 2}$.

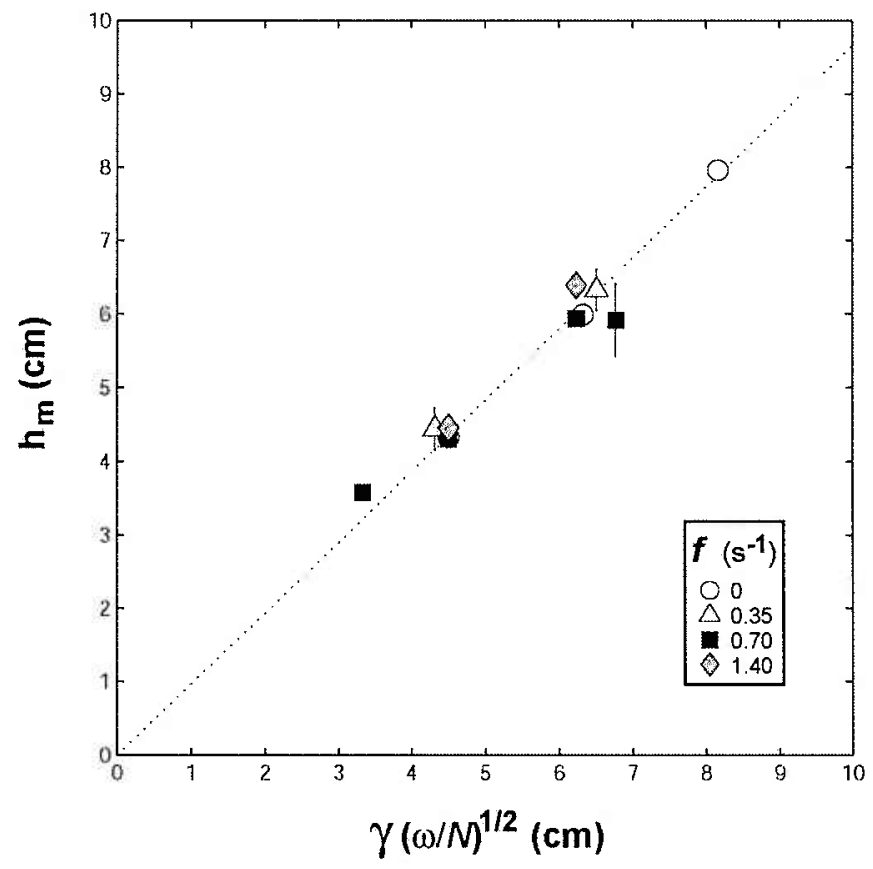

Figure 4.9: $\quad$ Mean height of mixed layer versus buoyancy scale height (3.13), for experiments with $7 \leq \omega \leq 28 \mathrm{~s}^{-1}, \quad 0.33 \leq N \leq 0.75 \mathrm{~s}^{-1}, 0 \leq f \leq 1.40 \mathrm{~s}^{-1}$, and $L_{B}=30.5 \mathrm{~cm}$. Symbols indicate rotation rates for individual experiments.

\section{3.c Advance of the mixed layer front}

The horizontal distribution of the mixed fluid in the rotating stratified experiments is investigated through two measurements: the position of the mixed layer front along an axis normal to the bar, and the total plan view area covered by dye. Figure 4.10 plots the 
advance of the mixed fluid along the normal axis as a function of time. The position is determined from the side view images and defined as the intrusion length, $l_{m}$, from the back of the oscillating bar to the tip of the advancing nose. The images in Figure 4.2 show a relationship between intrusion length and $N / f$. Therefore, length measurements are normalized by the deformation radius, $L_{R}=\frac{N}{f} h(3.15)$, with $h$ based on the layer height $h_{m}$ defined by equation (4.2). Time is scaled by $f^{-1}$. The intrusion advances approximately as $t^{2 / 3}$,

$$
l_{m}=(0.20 \pm 0.04) L_{R}(f t)^{0.65 \pm 0.04} .
$$

The time dependence may be attributed to the two-dimensional spreading of the intrusion as it advances outward from the bar. The rate of advance is proportional to $N h_{m}$, the speed of a gravity current with height $h_{m}$,

$$
\frac{d l_{m}}{d t}=0.13 N h_{m}(f t)^{-0.35} \text {. }
$$

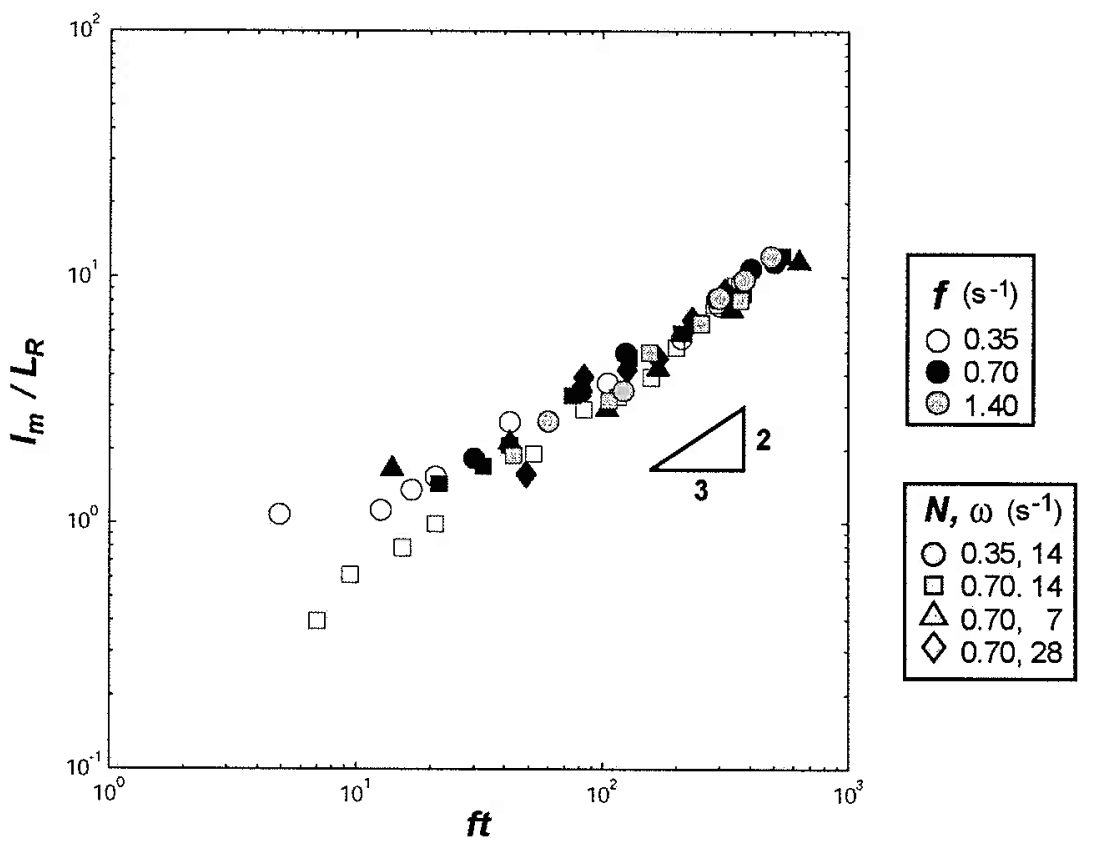

Figure 4.10: Advance of the mixed fluid along the normal axis. Intrusion length normalized by the deformation radius, $L_{R}=\frac{N}{f} h_{m}$, versus nondimensional time $f t$, for all rotating, stratified experiments with $L_{B}=30.5 \mathrm{~cm}$. 
The measurements of intrusion length are taken until the mixed outflow becomes irregular. The advancing front does not form into a boundary current or a distinct geostrophic rim current, but it does display a cyclonic and anticyclonic circulation. The flow at its leading edge develops baroclinic instabilites at intrusion length scales that equal a constant multiple of the deformation radius. Figure 4.11 shows the relationship in a plot of intrusion length, measured at the onset of instability, versus deformation radius,

$$
l_{m}=(10.3 \pm 1.7) L_{R}
$$

The first disturbances appear in the emergence of tendrils of dyed fluid or the formation of multiple lobes. Beyond this point, as seen in the images of Figure 4.4, the continued outflow of mixed fluid involves eddying motions that transport fluid further into the interior.

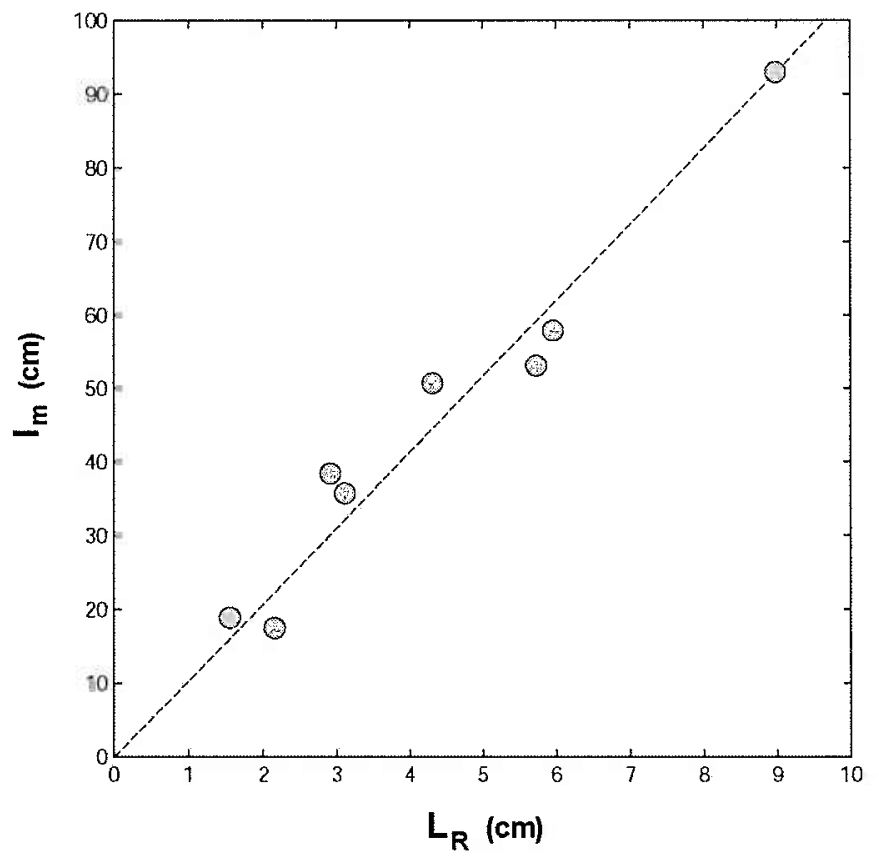

Figure 4.11: Position of the mixed fluid along the normal axis at the onset of instability. Length of intrusion versus deformation radius, $L_{R}=\frac{N}{f} h_{m}$, for all rotating, stratified experiments with $L_{B}=30.5 \mathrm{~cm}$. 


\section{3.d Lateral spreading of the mixed layer}

Prior to the development of instabilities, the advancing front exhibits a simple geometry, spreading smoothly outward from the mixing bar. Figure $4.12 a$ plots the dimensional measurements of area vs time, and Figure $4.12 b$ replots the data, with the area normalized by the deformation radius squared, $L_{R}^{2}$, and time normalized by $f^{-1}$. The data nondimensionalized in this way show considerable scatter and have a correlation coefficient $r=0.91$.
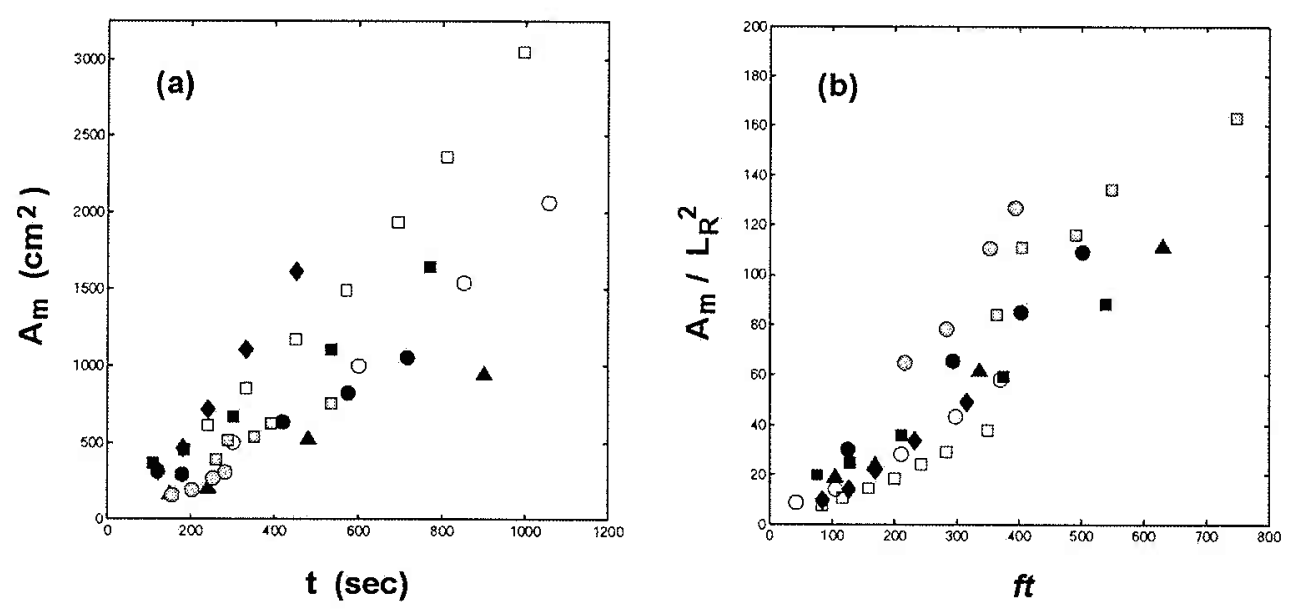

$\begin{array}{ll}f & \left(s^{-1}\right) \\ 0 & 0.35 \\ 0.70 \\ 0 & 1.40\end{array}$

$N, \omega\left(s^{-1}\right)$

$0.35,14$

$\square 0.70 .14$

$\triangle 0.70,7$

$\diamond 0.70,28$

Figure 4.12: Horizontal area of mixed fluid for all rotating, stratified experiments with $L_{B}=30.5 \mathrm{~cm}$. Symbols indicate values of $\omega, N$ and $f$. (a) Area $\left(\mathrm{cm}^{2}\right)$ vs time (sec), (b) Area normalized by $\mathrm{L}_{\mathrm{R}}{ }^{2}$ vs nondimensional time $f t$, with $L_{R}=\frac{N}{f} h_{m}$.

A better fit is found when the area is scaled by the product of $L_{R}{ }^{2}\left(\frac{f}{N}\right)^{1 / 2}$. The data, shown in Figure 4.13, now collapse to a straight line with a correlation coefficient $r=0.99$. The area of mixed fluid,

$$
A_{m}=(0.15 \pm 0.03) L_{R}{ }^{2}\left(\frac{f}{N}\right)^{1 / 2} f t .
$$


A physical interpretation of this empirical relation is suggested when the scale factors are rearranged as $L_{R}$ and $L_{R}\left(\frac{f}{N}\right)^{1 / 2}$. The second factor, rewritten using Equations (3.13) and (3.15), is the rotational length scale, (3.14),

$$
L_{R}\left(\frac{f}{N}\right)^{1 / 2}=\gamma\left(\frac{\omega}{f}\right)^{1 / 2} \equiv L_{f}
$$

Scaling the area by $\left(L_{R} L_{f}\right)$ suggests that the horizontal spreading of mixed fluid is more than an adjustment of laminar outflow to background rotation. It implies that the characteristics of the mixed layer are determined, in part, by the rotational control of turbulent motion.

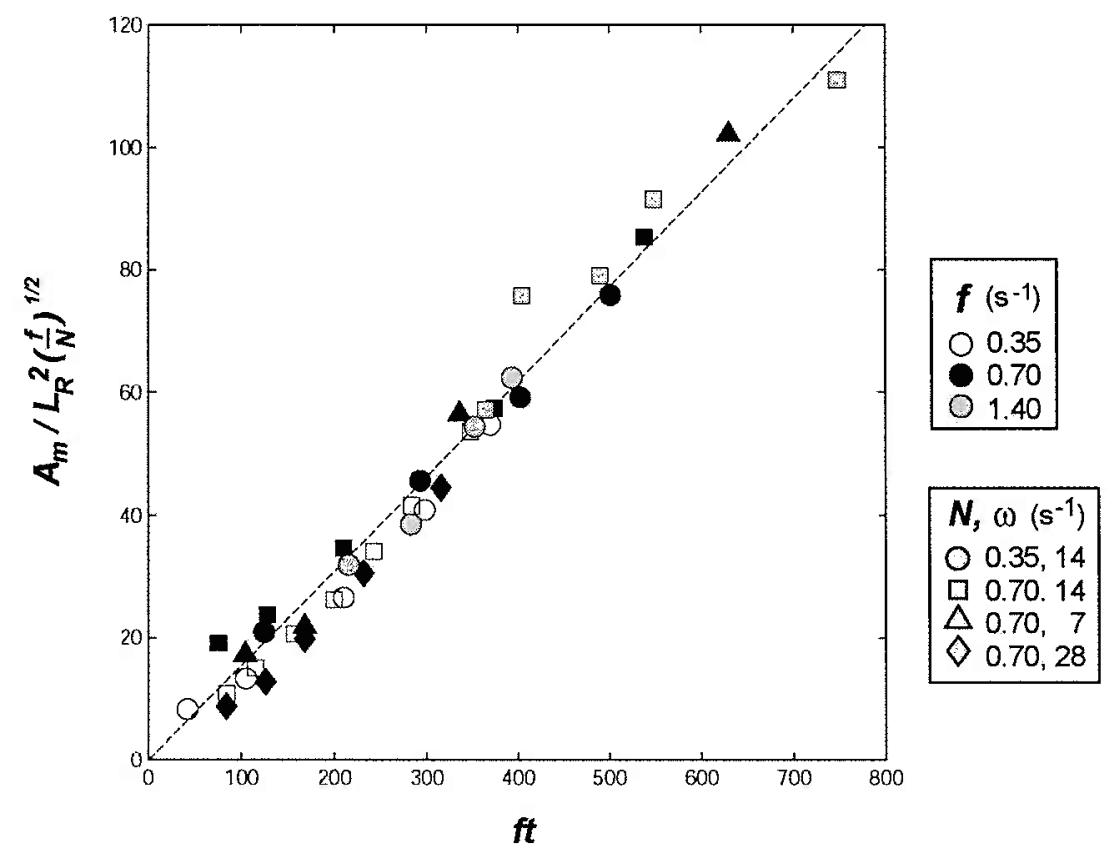

Figure 4.13: Horizontal area of mixed fluid for all rotating, stratified experiments with $L_{B}=30.5 \mathrm{~cm}$. Symbols indicate values of $\omega, N$ and $f$. Area normalized by $L_{R}{ }^{2}\left(\frac{f}{N}\right)^{1 / 2}$, with $L_{R}=\frac{N}{f} h_{m}$, vs nondimensional time $f$. 
Rotational control of bar-generated turbulence in the absence of stratification is examined separately in a set of three experiments, with $\omega=14 \mathrm{~s}^{-1}$ and $f=[0.35,0.70,1.40] \mathrm{s}^{-1}$. The dye injected at the bar does not capture the transition from isotropic turbulence to rotationally dominated flow. The dye immediately forms into vertical sheets which span the fluid depth and expand horizontally into the undisturbed interior. Figure 4.14 plots the growth of the dyed regions and shows the relationship between the area and the rotational length scale, $L_{f}$,

$$
A=(0.09 \pm 0.02) L_{f}^{2} f t \text {. }
$$

The experimental results, although limited, indicate that the horizontal scale of motion in a homogeneous fluid is consistent with rotational inhibition of turbulence.

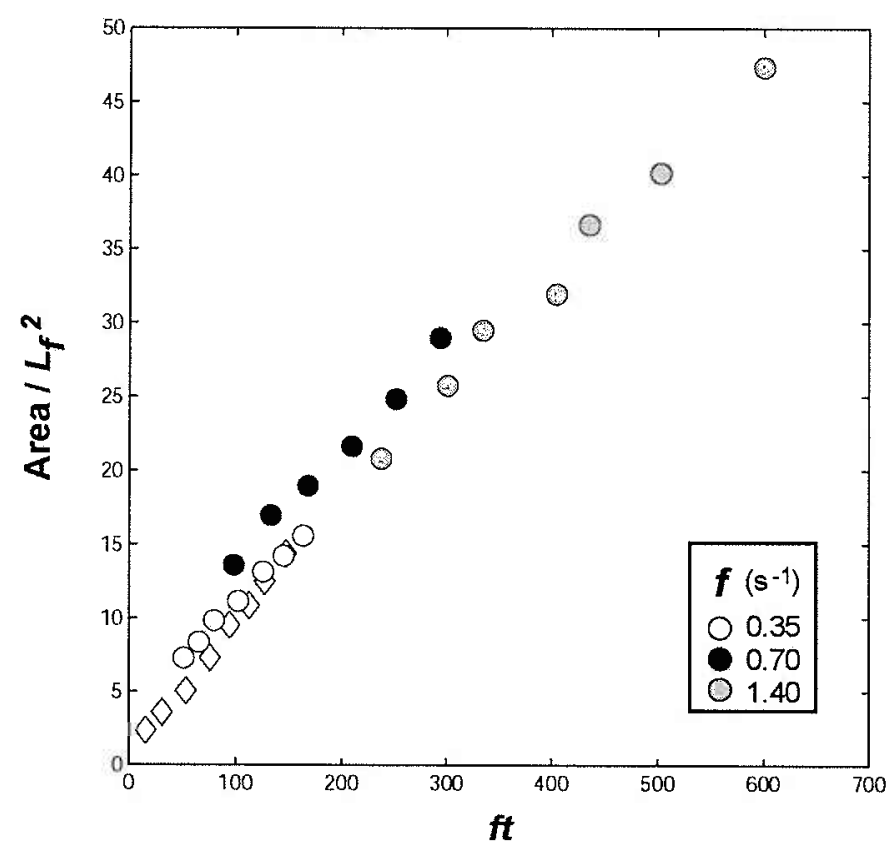

Figure 4.14: Horizontal area of dyed fluid for unstratified, rotating experiments. Area normalized by the rotational length scale, $L_{f}(3.14)$, vs nondimensional time $f t$. The circles indicate experiments with $L_{B}=30.5 \mathrm{~cm}$. The diamonds indicate an experiment with $L_{B}=15.2 \mathrm{~cm}$, (see section 4.4.c).

In the stratified experiments, rotation also appears to influence the horizontal scales of turbulent motion. Section 4.4 proposes a model of turbulent entrainment which relates both rotation and stratification to the volume flux of mixed fluid. 


\section{3.e Volume of mixed fluid}

The volume of mixed fluid is defined as the product of the mean observed height $h_{m}$ and the temporally evolving area $A_{m}$, and is documented from $\sim 150$ seconds onward. Figure 4.15 plots volume, normalized by $L_{R} L_{f} h_{m}$, vs the nondimensional time $f t$. This scaling implies that the volume is independent of the mixing bar length. The data describe an approximately linear increase in the volume of mixed fluid.

$$
V_{m}=(0.16 \pm 0.03) L_{R} L_{f} h_{m} f t .
$$

The volume flux of mixed fluid is

$$
\frac{d V_{m}}{d t}=Q=0.16 L_{R} L_{f} h_{m} f
$$

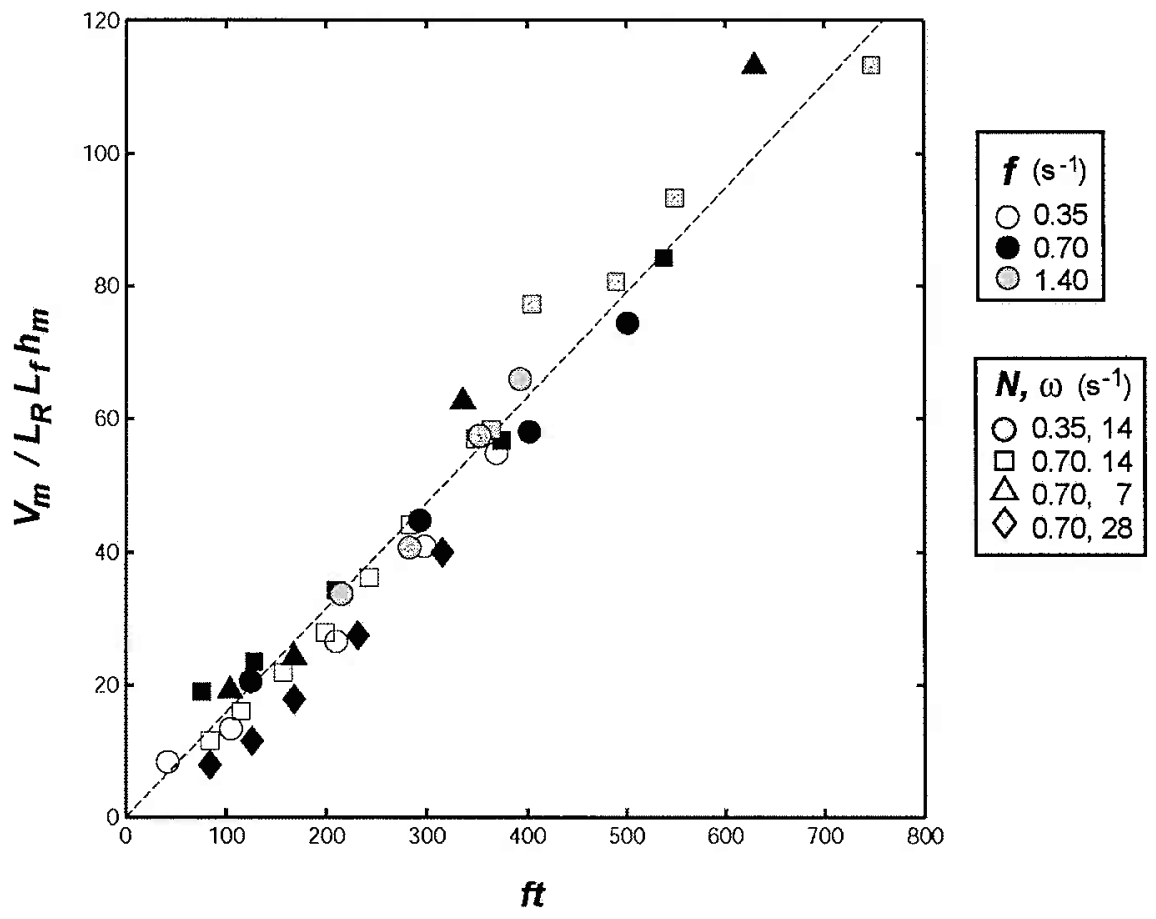

Figure 4.15: Volume of mixed fluid for all rotating, stratified experiments with $L_{B}=30.5 \mathrm{~cm}$. Symbols indicate values of $\omega, N$ and $f$. Volume normalized by $L_{R} L_{f} h_{m}$ vs nondimensional time $f t$, with $L_{R}=\frac{N}{f} h_{m}$, and $L_{f}$ and $h_{m}$ given by (3.14) and (4.2), respectively. 


\subsection{A model for mixing in the presence of rotation}

\section{4.a Model of lateral entrainment}

An entrainment model is proposed that provides a physical interpretation for the volume flux of exported mixed fluid. The basic features of the model are contained in Figure 4.16 which sketches export of mixed fluid from the front of the turbulent zone and entrainment of unmixed fluid into the sides.

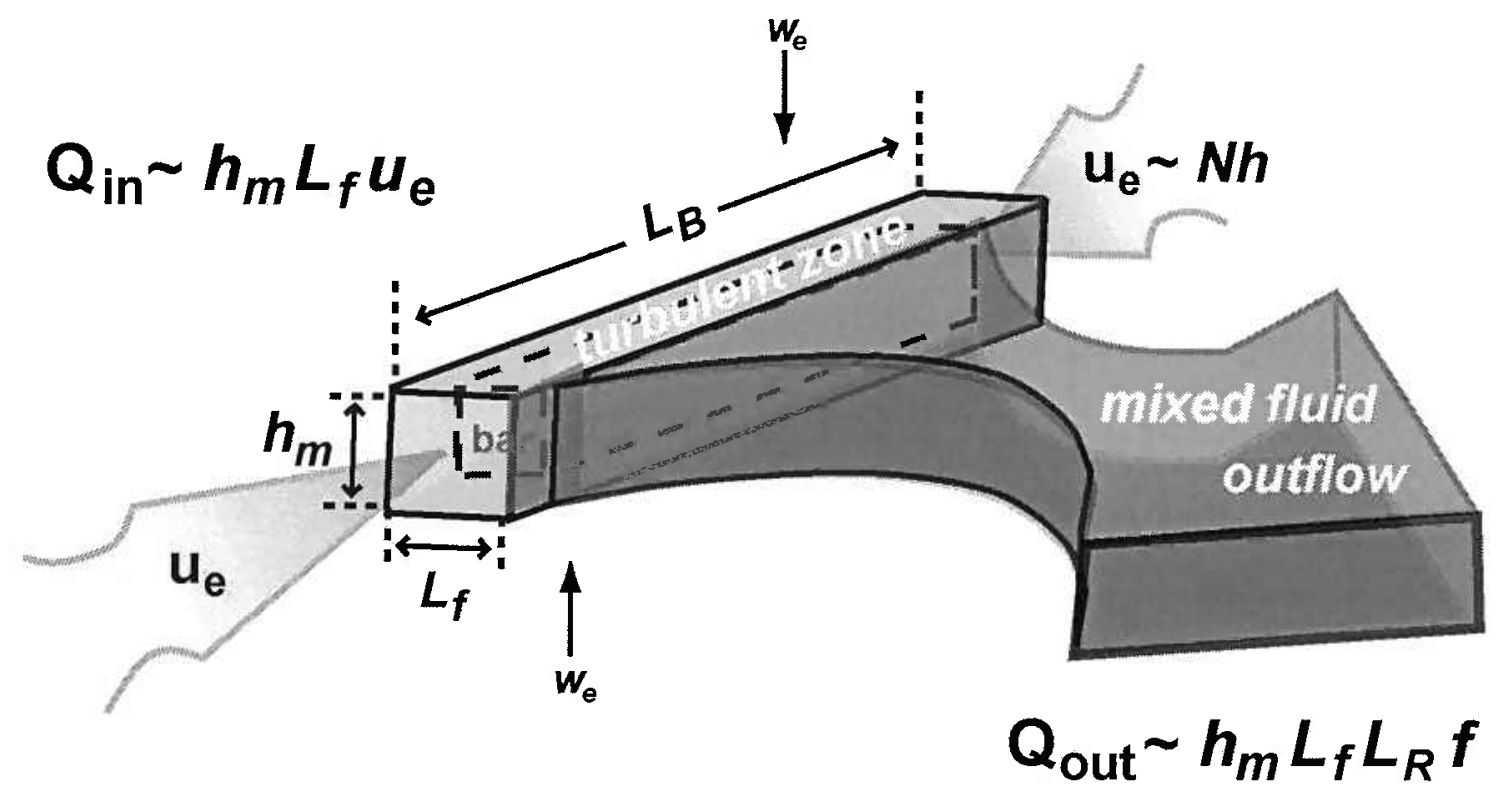

Figure 4.16: A schematic diagram showing the outward flux of mixed fluid, and the lateral entrainment of stratified fluid at the sides of the turbulent zone. The dimensions of the turbulent zone are labelled, $L_{B} \times L_{f} \times h_{m}$, where $L_{B}$ is the bar length and $L_{f}$ and $h_{m}$ given by (3.14) and (4.2), respectively.

The basic assumptions of the model are as follows.

(a) The size of the turbulent zone is set by the bar length, and the ambient stratification and rotation. The height is proportional to the buoyancy height scale $H_{N}$ (3.13), and specifically, the measured layer height $h_{m}(4.2)$. The length is approximately equal to the bar length $L_{B}$, and the width is proportional to the rotational length scale $L_{f}(3.14)$. The 
images of layer height (Figure $4.2 a, b$ ) and of mixed fluid outflow (Figure $4.3 a, b$; Figure 4.4 a) support the assumptions about the mixing region height and length .

(b) Nonturbulent fluid is entrained into the turbulent zone both vertically and horizontally, but vertical entrainment is negligible. This assumption is discussed more fully in the next section.

(c) Horizontal entrainment of stratified fluid occurs at the sides of the turbulent zone over a length scale $L_{f}$. The cross tank dye line experiments (Figure 4.6) illustrate an outward flux from the face of the bar and inward flows at the sides.

(d) The entrainment velocity $u_{e}$ is proportional to $N h_{m}$. This is consistent with an interior flow in thermal wind balance over a length scale comparable to the deformation radius $L_{R}$ (3.15).

As sketched in Figure 4.16, ambient fluid enters across a surface area, $h_{m} \times L_{f}$, with a velocity $u_{e}$ giving an influx,

$$
Q_{\text {in }} \propto h_{m} L_{f} u_{e} .
$$

The lateral influx must equal the outflow, thus

$$
Q_{\text {out }} \propto h_{m} L_{f} L_{R} f,
$$

where $L_{R} f=N h_{m}$. This agrees with the experimental result (4.10), and interestingly, is independent of the mixing zone length, $L_{B}$.

\section{4.b Model of vertical entrainment}

An alternative hypothesis is that production of mixed fluid occurs through vertical entrainment only. The assumptions for this model follow:

(a) The size of the turbulent zone is set by the bar length and the effects of the ambient rotation and stratification. As in the previous model, the dimensions are given by: $L_{B} \times L_{f} \times h_{m}$

(b) Horizontal entrainment is considered negligible. 
(c) Vertical entrainment of unmixed fluid occurs across the upper and lower surfaces of the turbulent zone. Section 4.3.a describes the rapid establishment of a mixed layer of height $h_{m}$, given by equation (4.2), and Figure 4.7 shows the increasingly sharp interface between the mixed layer and the ambient stratification. The model is therefore based on entrainment across a density interface, and considers entrainment for times $t \geq 100 \mathrm{~s}$, after the height $h_{m}$ has been set.

(d) The vertical entrainment velocity, $w_{e} \propto\left(N h_{m}\right)(N t)^{-7 / 8}$. This relation is consistent with the results of the small tank experiments reported in Chapter 3.3, where, in the absence of rotation, the longterm growth of the turbulent zone height went as $h \sim\left(\frac{K}{N}\right)^{1 / 2}(N t)^{1 / 8}$. It is also consistent with the studies of Linden (1973), Maxworthy and Monismith (1988), Fleury et al. (1991) and others, which support entrainment laws of the form, $R i^{-3 / 2}$, where $R i$ is the Richardson number defined by equation (2.7).

The calculation of vertical entrainment for the single-bar experiments employs the constant of proportionality empirically determined by Fleury et al. for nonrotating stratified fluids, (see equation 2.34). Fleury et al. also developed a formula for rotating fluids and demonstrated that rotation inhibited growth for $\frac{N}{f}<3$. The nonrotating formula is used here because it provides a maximum estimate for vertical entrainment. Following the methodology outlined in Chapter 2.3.b, $w_{e}$ is defined as the displacement of the density interface over time and expressed as a function of $N t$. With the parameterizations of bar-generated turbulence (3.5) and (3.6), the Fleury et al. (1991) relation becomes

$$
w_{e} \equiv \frac{d z}{d t}=0.11 N h_{m}(N t)^{-7 / 8},
$$

where $z$ is the distance between bar centerline and interface. According to (4.13) and the assumptions of the vertical entrainment model, vertical flux across the top and bottom surfaces is given by

$$
Q_{\text {vert }}=0.22\left(L_{B} L_{f} N h_{m}\right)(N t)^{-7 / 8} \text {, }
$$


which implies an equivalent outflux that is a function of mixing region length, $L_{B}$.

Total influx is calculated for the period that extends from the establishment of the mixed layer height at $t \approx 100 \mathrm{~s}$ to the limit of volume measurements at $t \approx 1000 \mathrm{~s}$. The model is applied to two cases. The experiment with the maximum possible vertical entrainment has $(\omega, N, f)=(28,0.61,0.70) \mathrm{s}^{-1}$ and $L_{B}=30.5 \mathrm{~cm}$. The total volume change is calculated as

$$
\int_{100 \mathrm{~s}}^{1000 \mathrm{~s}} Q d t=1300 \mathrm{~cm}^{3} .
$$

An experiment with more typical parameters, $(\omega, N, f)=(14,0.70,0.70) \mathrm{s}^{-1}$ and $L_{B}=30.5 \mathrm{~cm}$, shows a volume change of

$$
\int_{100 \mathrm{~s}}^{1000 \mathrm{~s}} Q d t=600 \mathrm{~cm}^{3} .
$$

The observed volume growth during the same period is $25500 \mathrm{~cm}^{3}$ and $9000 \mathrm{~cm}^{3}$ for the two cases. Thus, vertical entrainment accounts for only $5 \%$ and $7 \%$ of the respective totals.

However, the assumption that $w_{e} \sim t^{-7 / 8}$ may be inappropriate. Entrainment velocity depends on the distance between the source of turbulent mixing, the bar, and the unmixed fluid. Although the density interface is sharpening during the observation period, the overall height of the turbulent zone remains approximately uniform.

A modified model assumes that the curtailment of vertical entrainment is coincident with establishment of mixed layer height, but, once reduced, the entrainment rate remains constant as long as layer height is unchanged. A rough estimate of volume change is made by calculating the vertical flux (4.14) during the first ten seconds after layer height has been established. The result is then multiplied by 90 to cover the entire $900 \mathrm{~s}$ period. For the experiment with maximum flux, the volume growth is

$$
\left(90 \times \int_{100}^{110 \mathrm{~s}} Q d t\right)=4100 \mathrm{~cm}^{3} \text {, }
$$


while for the typical experiment it is

$$
\left(90 \times \int_{100 \mathrm{~s}}^{110 \mathrm{~s}} Q d t\right)=2000 \mathrm{~cm}^{3} .
$$

By this calculation, vertical entrainment equals $16 \%$ and $22 \%$ of total observed outflows. It does not account for the total production of mixed fluid. However, if this fraction of mixed fluid is due to vertical entrainment, then changes in the bar length, $L_{B}$, may significantly affect the production.

\section{4.c Variations in bar length}

In the original set of experiments the bar length is a constant, $L_{B}=30.5 \mathrm{~cm}$. The observed volume of mixed fluid, described by equation (4.9), and the lateral entrainment model imply that the production of mixed fluid is independent of bar length. This conclusion is examined through a series of experiments with $L_{B}=[15.2,20.3,22.9] \mathrm{cm}$, $\frac{1}{2}, \frac{2}{3}$, and $\frac{3}{4}$ of the original length, respectively. The range of bar size is based on two considerations: keeping the mixing region small relative to the tank size, and keeping it long relative to the rotational length scale, $L_{f}$, in order to avoid interactions between lateral flows being entrained into the turbulent zone from opposite sides. In all cases the length is much greater than the bar height and the amplitude of bar motion, and it is assumed that the properties of turbulent motion, given by (3.5) and (3.6), are unchanged.

For each bar length, experiments are conducted with three different values of $\frac{N}{f}$. The Coriolis and buoyancy frequencies are varied, with $0.30 \leq f \leq 1.40 \mathrm{~s}^{-1}$ and $0.33 \leq N \leq 0.83 \mathrm{~s}^{-1}$, though usually $N \approx 0.7 \mathrm{~s}^{-1}$. The bar frequency has a constant value, $\omega=14 \mathrm{~s}^{-1}$. As in the original set of experiments, volume is calculated from the product of the horizontal area and the mean mixed layer height for each experiment.

Figure 4.17 plots the mean heights versus the buoyancy height scale $H_{N}$ (3.13). The new data is superimposed on the first data set, and falls within the error limits of the 
original fit, $h_{m}=(0.97 \pm 0.05) \gamma\left(\frac{\omega}{N}\right)^{1 / 2}$ (4.2). The unchanged relationship to the scale height suggests that turbulent properties are unaffected by the variation in bar length.

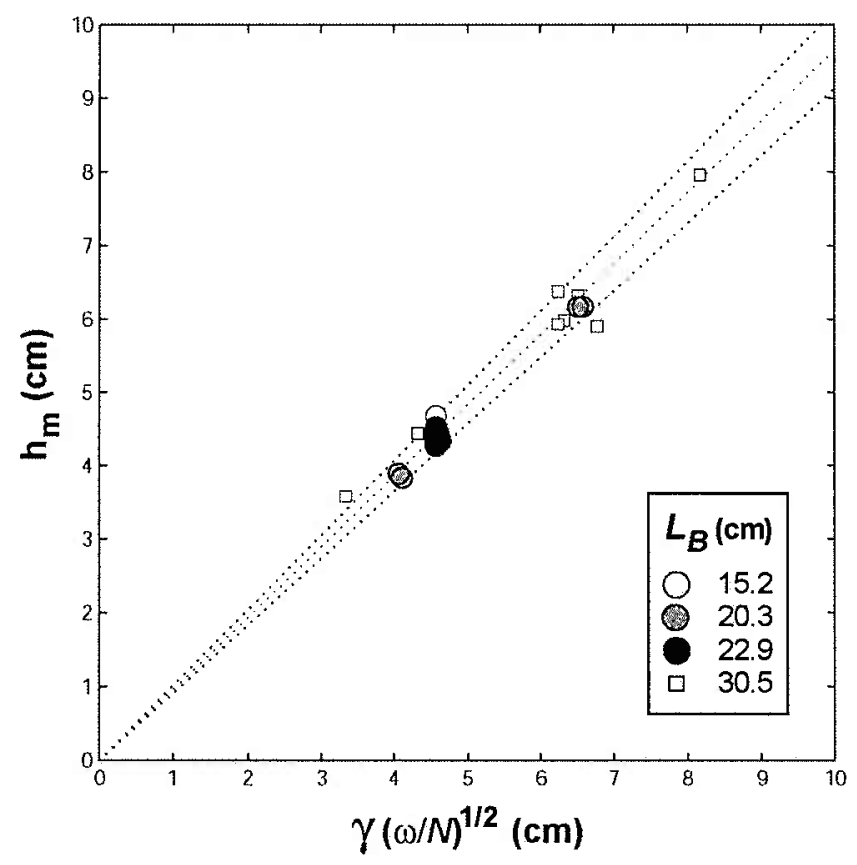

Figure 4.17: Mean height of mixed layer versus buoyancy scale height (3.13), for experiments with all bar lengths. The small squares indicate the original set with $L_{B}=30.5 \mathrm{~cm}$ (see Figure 4.9). The dotted lines give the original straight line fit \pm one standard deviation.

The rotational length scale $L_{f}$ (3.14), which also depends on the turbulent properties, is examined in an unstratified experiment using the shortest bar, $L_{B}=15.2 \mathrm{~cm}$, with $(\omega, f)=(14,0.35) \mathrm{s}^{-1}$. The measurements of areal spreading are included in Figure 4.14, and scale as $L_{f}^{2}(4.8)$, consistent with the original unstratified data for $L_{B}=30.5 \mathrm{~cm}$.

Volume calculations from the nine rotating, stratified experiments are presented in Figure 4.18. Panel $a$ plots the dimensional data, and panel $b$ plots the data scaled according to (4.9). The results from the experiments with the $\frac{3}{4}$ length bar are on the low 
side, but there is no pattern of smaller bar lengths generating smaller volumes of mixed fluid. Overall, the best fit for all bar lengths is the original scaling given by equation (4.9).
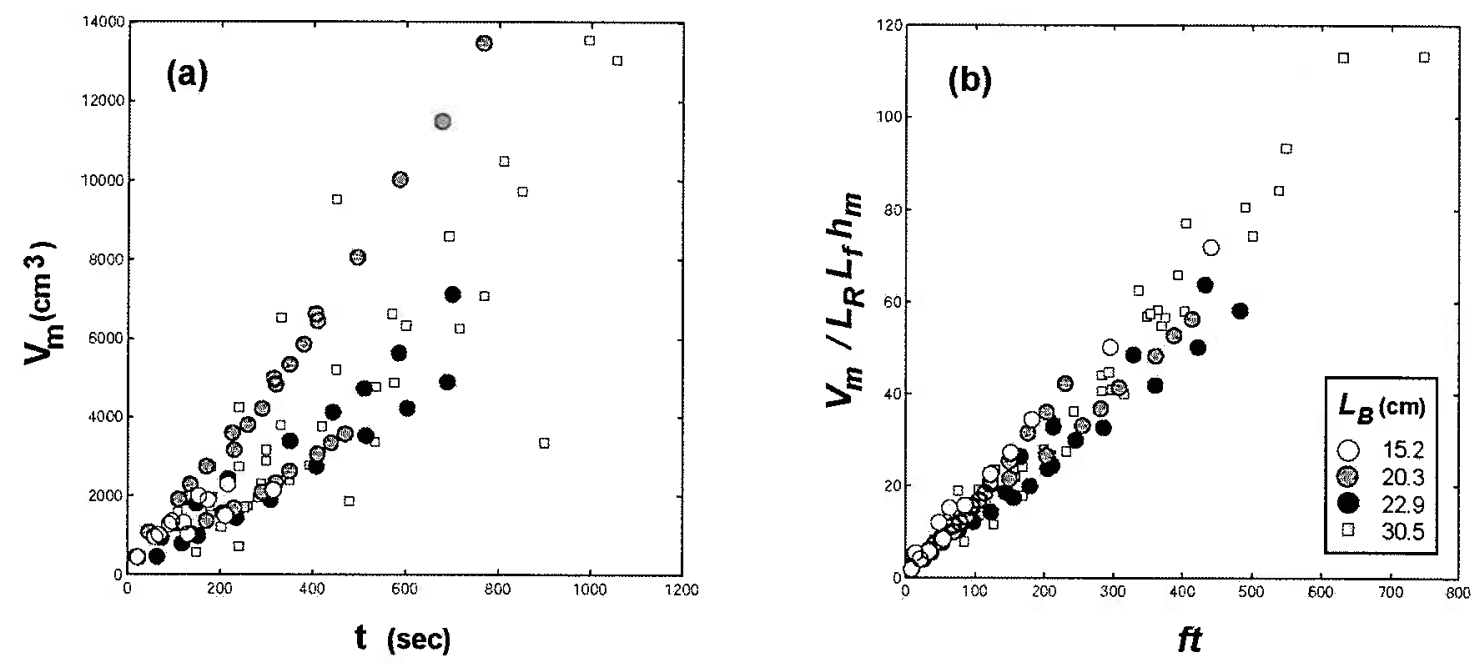

Figure 4.18: Volume of mixed fluid for all bar lengths. The small squares indicate the original set with $L_{B}=30.5 \mathrm{~cm}$ (see Figure 4.15). (a) Volume $\left(\mathrm{cm}^{3}\right)$ vs time (sec), (b) volume normalized by $L_{R} L_{f} h_{m}$ vs nondimensional time $f t$, with $L_{R}=\frac{N}{f} h_{m}$, and $L_{f}$ and $h_{m}$ given by (3.14) and (4.2), respectively

\subsection{The limits of rotational effects}

The experiments discussed so far have investigated localized mixing in rotating, stratified fluids with $\frac{1}{4} \leq \frac{N}{f} \leq 2$. They indicate that while local vertical mixing and the height of the mixed layer are independent of rotation (4.2), the production of mixed fluid over time is restricted by it. The restrictive effects of rotation are seen in the lateral spreading (4.6) and the volume growth (4.9) of mixed fluid, both of which go as $f^{-1 / 2}$. The flux of mixed fluid increases as the rotation rate decreases, but flux cannot go to infinity as the rotation rate goes to zero. This section looks at the limit, $f=0$, in order to investigate 
behavior of mixed fluid production as $f \rightarrow 0$. The results from six stratified nonrotating experiments are presented. Three experiments are conducted for the original bar length, $L_{B}=30.5 \mathrm{~cm}$, with $N=[0.21,0.35,0.69] \mathrm{s}^{-1}$, and one each for the other bar lengths $L_{B}=[15.3,20.3,22.9] \mathrm{cm}$ and $N=[0.67,0.85,0.67] \mathrm{s}^{-1}$, respectively.

The mean mixed layer heights $h_{m}$ are measured near the turbulent zone. The data are included in the plots in Figures 4.9 and 4.17, and are indistinguishable from the results of the rotating experiments. However, the layers do not maintain uniform heights as they move into the interior. In the absence of rotation, the turbulent fluid simply collapses into a thinning intrusion of mixed fluid as seen in the shadowgraph in Figure $3.3 b$.

The horizontal areas of the spreading fluid are measured as before from the plan views, but the volume estimates are complicated by the nonuniform heights. The volume is estimated using a mean height $\bar{h}$, calculated from the intrusion side view area and length. This method tends to overestimate the volume when the mixed layer spreads beyond the width of the mixing bar, but other methods, such as fitting triangular and parabolic functions to the intrusion shape, were inconsistent predictors of layer height.

The volume estimates are shown in Figure 4.19. Panel $a$ plots the dimensional data, and panel $b$ shows the volume scaled by $L_{B} h_{m}{ }^{2}$ versus nondimensional time $N t$. This scaling is the best fit for all the data, even though the data from the experiment with the $\frac{3}{4}$ bar length falls below the others, just as it does in the rotating experiments. The experiments for each bar length involve separate laboratory setups and it is possible that different conditions have been unintentionally introduced. If the $\frac{3}{4}$ bar experiment is excluded, a straight line fit of the data gives the volume,

$$
V_{m}=(0.072 \pm 0.008) L_{B} h_{m}{ }^{2} N t .
$$

With all data included, the volume goes as $V_{m}=(0.064 \pm 0.011) L_{B} h_{m}{ }^{2} N t$. The flux $Q$ is constant,

$$
Q_{f=0} \propto L_{B} h_{m} N h_{m}
$$



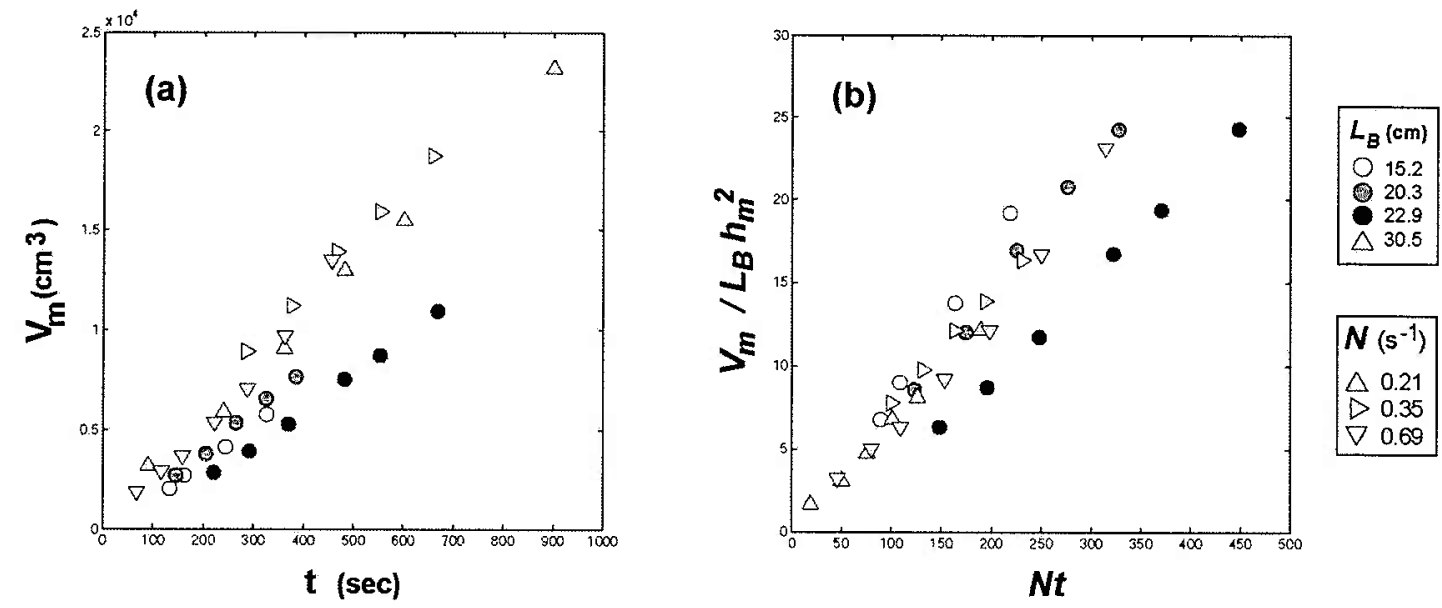

Figure 4.19: Volume of mixed fluid in experiments without rotation. Bar length, $L_{B}$, is indicated by shading. The stratification in the experiments with $L_{B}=30.5 \mathrm{~cm}$ is indicated by the triangular shapes. (a) Volume $\left(\mathrm{cm}^{3}\right)$ vs time (sec), (b) volume normalized by $L_{B} h_{m}{ }^{2}$ vs nondimensional time $N t$.

The production of mixed fluid may be driven by the horizontal pressure gradient at the face of the turbulent zone, of length $L_{B}$ and height $h_{m}$, and supplied by a combination of vertical and horizontal entrainment. The vertical entrainment model (section 4.4.b) suggests that vertical entrainment alone is insufficient. The qualitative observations of circulation in the nonrotating experiments indicate the presence of lateral entrainment. Dye traces from potassium permanganate crystals show compensatory return flows of ambient fluid at the level of the mixed layer. These flows may also be supplemented by horizontal flow into the front of the turbulent region along the top and bottom edges of the collapsing intrusion, as described in the experiments of De Silva and Fernando (1998).

Figure 4.20 compares the production of mixed fluid with and without rotation. The flux calculated for each experiment is scaled by $L_{B} h_{m} N h_{m}$. The rotating experiments are plotted versus $L_{f} / L_{B}$. Values for the nonrotating experiments, which correspond to $L_{f} \rightarrow \infty$, are placed at the far right of the plot. For a given bar length, the flux of mixed fluid in the presence of rotation is always lower than for $f=0$. The magnitude of flux 
approaches the nonrotating values as the rotational length scale $L_{f}$ increases, that is, as rotation decreases. The limiting value is $L_{f} / L_{B} \sim 0.4-0.5$. When the rotational length scale approaches the half-length of the mixing region, the limiting factor in the production of mixed fluid may be the length over which the mixed fluid can be exported from the turbulent zone rather than length over which it can be laterally entrained. This could imply that the production of mixed fluid is capped by the nonrotating relation (4.22). However, there is no data for $L_{f} / L_{B}>0.5$. It is interesting that the factor that differentiates rotating from nonrotating experiments is a function of the rotational length scale and the length of the mixing region rather than a function of $N / f$. For ratios where $0.5 \leq \frac{L_{f}}{L_{B}}<1$, fluid entrained into the sides of the turbulent region, over scales $\sim L_{f}$, may interact and drive a jet of mixed, or partially mixed, fluid into the interior and possibly produce an even greater volume of mixed fluid than in the absence of rotation.

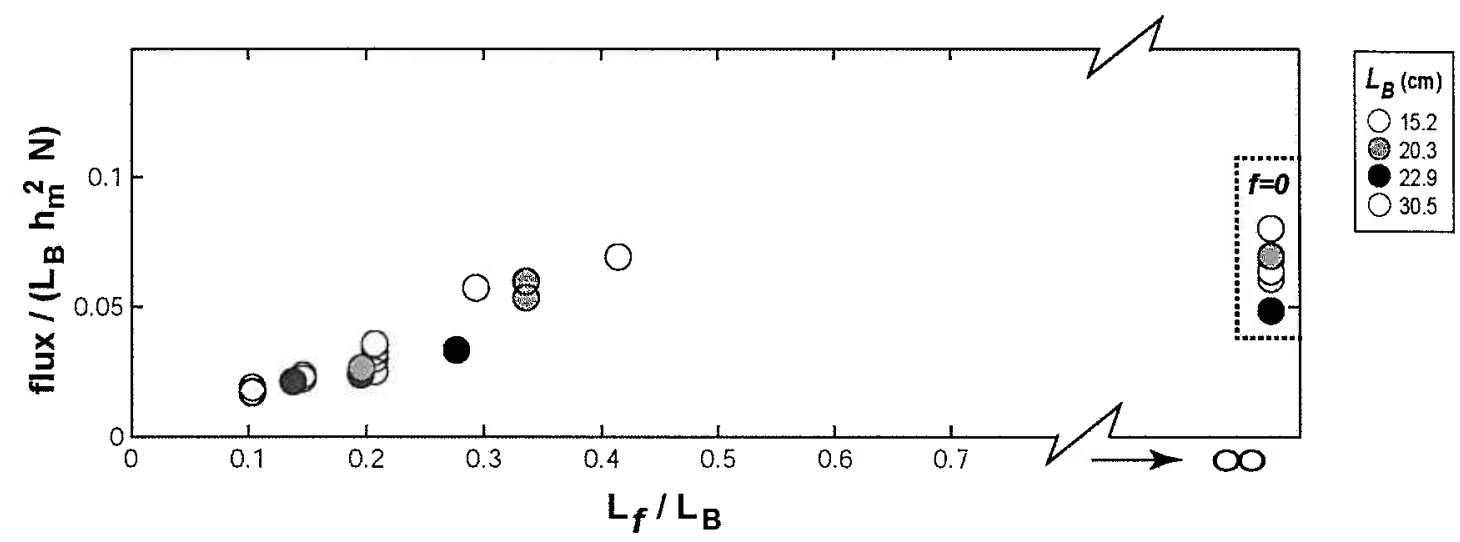

Figure 4.20 Flux of mixed fluid in experiments with and without rotation. The legend indicates the bar length $L_{B}$ of each experiment. For the rotating experiments, flux is normalized by $L_{B} h_{m}{ }^{2} N t$ and plotted vs $L_{f} / L_{B}$, with $L_{f}$ and $h_{m}$ given by (3.14) and (4.2), respectively. Flux for the experiments without rotation is plotted on the far right. 


\subsection{Discussion}

The laboratory study, described in this chapter, investigates the interaction between a patch of vertical boundary mixing and a body of rotating stratified fluid. The experimental results, which cover the parameter range $\frac{1}{4} \leq N / f \leq 2$, illustrate the contribution of $N$ and $f$ to the production and distribution of mixed fluid, and also bring out the importance of geometric features, such as the exposed sides of the mixing region and the overall length of the mixing bar.

The laboratory measurements of mixed layer height, lateral area, and total volume have been evaluated in terms of three basic length scales, $H_{N}$ (3.13), $L_{f}$ (3.14) and $L_{R}$ (3.15), each of which implies an aspect of the underlying physics of mixing. The first two, the buoyancy and rotational length scales, are related to the interaction of turbulence with the ambient fluid, and the third, the deformation radius, is related to the adjustment of the mixed fluid outflow to background rotation. The empirical relationships, when rewritten in terms of the external parameters, $N, f$ and $\omega$, highlight the role of the buoyancy and Coriolis frequencies.

The mixed layer height, which is measured near the bar, is quickly established and remains nearly steady over time. It is a function of the ambient stratification and independent of rotation,

$$
h_{m} \sim \omega^{1 / 2} N^{-1 / 2}
$$

The lateral spreading of mixed fluid increases with stratification, decreases with greater rotation, and is linear in time,

$$
A_{m} \sim \omega\left(\frac{N}{f}\right)^{1 / 2} t
$$

The volume, $V_{m}=h_{m} A_{m}(t)$, also grows linearly with time. It is restricted by rotation and, interestingly, is independent of the stratification.

$$
V_{m} \sim \omega^{3 / 2} f^{-1 / 2} t
$$


The characteristic dimensions of the mixed fluid, whether set by rotation, stratification or both, are all also functions of the intensity of the imposed mixing, which is represented by the bar frequency $\omega$ (see Chapter 3.3). This parameterization of bargenerated turbulence, while useful in evaluating the experimental results, is not directly transferable to oceanic mixing processes. However, the laboratory findings can be formulated without reference to the mixing parameter $\omega$, by making use of the robust relationship of mixed layer height to the buoyancy height scale, (4.2). The mixing intensity is implicit in the magnitude of the height. Thus the flux of locally mixed fluid is given by

$$
\frac{d V_{m}}{d t}=Q=0.16\left(\frac{N}{f}\right)^{3 / 2} h_{m}^{3} f
$$

and its lateral spreading is predicted by

$$
A_{m}=0.15\left(\frac{N}{f}\right)^{3 / 2} h_{m}^{2} f t
$$

In this form, the laboratory results may be useful in understanding the circulation of mixed fluid generated by a variety of mechanisms. In regions of intensified oceanic mixing, observations of mixed layer thickness, together with the local buoyancy and Coriolis frequencies, could indicate the magnitude of the mixed fluid production and the extent of its areal distribution.

In the abyssal ocean, where the buoyancy period is 3 to 5 hours, $1 \leq N / f \leq 10$, and in highly stratified regions of the upper ocean, the ratio can go as high as $N / f=O\left(10^{2}\right)$. Although most laboratory values of $N / f$ are lower than those encountered in the ocean, the experiments at the limit, $f=0$, suggest that $L_{f} / L_{B}$ rather than $N / f$ is the factor that determines whether or not rotational effects are significant.

The ratio of the rotational length scale $L_{f}$ to the bar length $L_{B}$ is related to the characteristics of the mixing region. The lateral entrainment model, developed in section 4.4 and sketched in Figure 4.16, describes the export of mixed fluid into the interior from 
the face of the bar and the entrainment of unmixed fluid at the sides. It hypothesizes that the presence of rotation restricts lateral entrainment on a scale proportional to $L_{f}$ and thus limits the production of mixed fluid. The physical interpretation is consistent with the observations of flow patterns reported in section 4.4 , and with the scaled relations for height (4.2), area (4.6) and volume (4.9), empirically developed in section 4.3. The experiments with bar variations (section 4.4.c) demonstrate that the production of mixed fluid is independent of bar length, at least within the test range, $0.1 \leq L_{f} / L_{B} \leq 0.4$, and thus they offer further support for a model of mixing that depends on lateral rather than vertical entrainment.

In the experiments with an exposed patch of mixing at a vertical boundary, many of the characteristics of mixed fluid production and distribution follow from the decisive role of lateral entrainment. Oceanic observations, however, often associate intensified mixing with abyssal canyons and rough topographic features that pose barriers to lateral entrainment. If the sides are blocked, the dynamics of mixing can change considerably. Mixed fluid production may shift from rotational control to dependence on mixing region length and vertical entrainment. Flow patterns can change as well. The presence of side walls may promote the formation of a boundary current or otherwise redirect flow into the interior. In the next chapter, these possibilities are addressed in a series of experiments with mixing imposed at the closed end of a channel that opens at its other end to a larger basin. 


\section{Chapter 5}

\section{Mixing in a Channel}

\subsection{Introduction}

Observations of spatially inhomogeneous mixing in the deep ocean motivated the vertical boundary mixing experiments of Chapter 4 . Those experiments address the question of fluid exchanges between an isolated turbulent patch and an open basin of rotating stratified fluid. The results indicate that horizontal entrainment of ambient fluid at the sides of the turbulent zone sets the limits of mixed fluid production and affects its export into the interior.

The observations that describe the uneven distribution of mixing in the ocean also identify intensified mixing rates with regions of rough bathymetry (Toole et al., 1994, 1997; Lueck and Mudge, 1997; Polzin et al., 1997; Ledwell et al., 2000). The topographic features associated with enhanced mixing, such as the crests and canyons of the transverse fracture zones of the MidAtlantic Ridge, may pose barriers to lateral entrainment and prevent the development of circulations similar to those seen in the laboratory experiments. This chapter reports a second set of experiments which examine the effects of blocking access to the sides of the mixing zone. In these experiments, the mixing bar is laterally confined between two walls.

Except for the two walls, the experimental configuration is unchanged. The rectangular tank and interior circular wall, the counterclockwise rotation, the linear salt- 
stratified fluid, and the single horizontal mixing bar are all identical to the vertical boundary mixing experiments of Chapter 4 and are fully described in Chapter 3. Figure 5.1 sketches the laboratory apparatus, as viewed by the overhead video camera. Two, $61 \mathrm{~cm}$ long, plexiglass walls are added, extending the full depth of the fluid beneath the rigid lid and abutting the sides of the mixing bar. The outer wall and the side walls enclose the mixing bar on three sides and create a channel which opens into the rest of the basin.

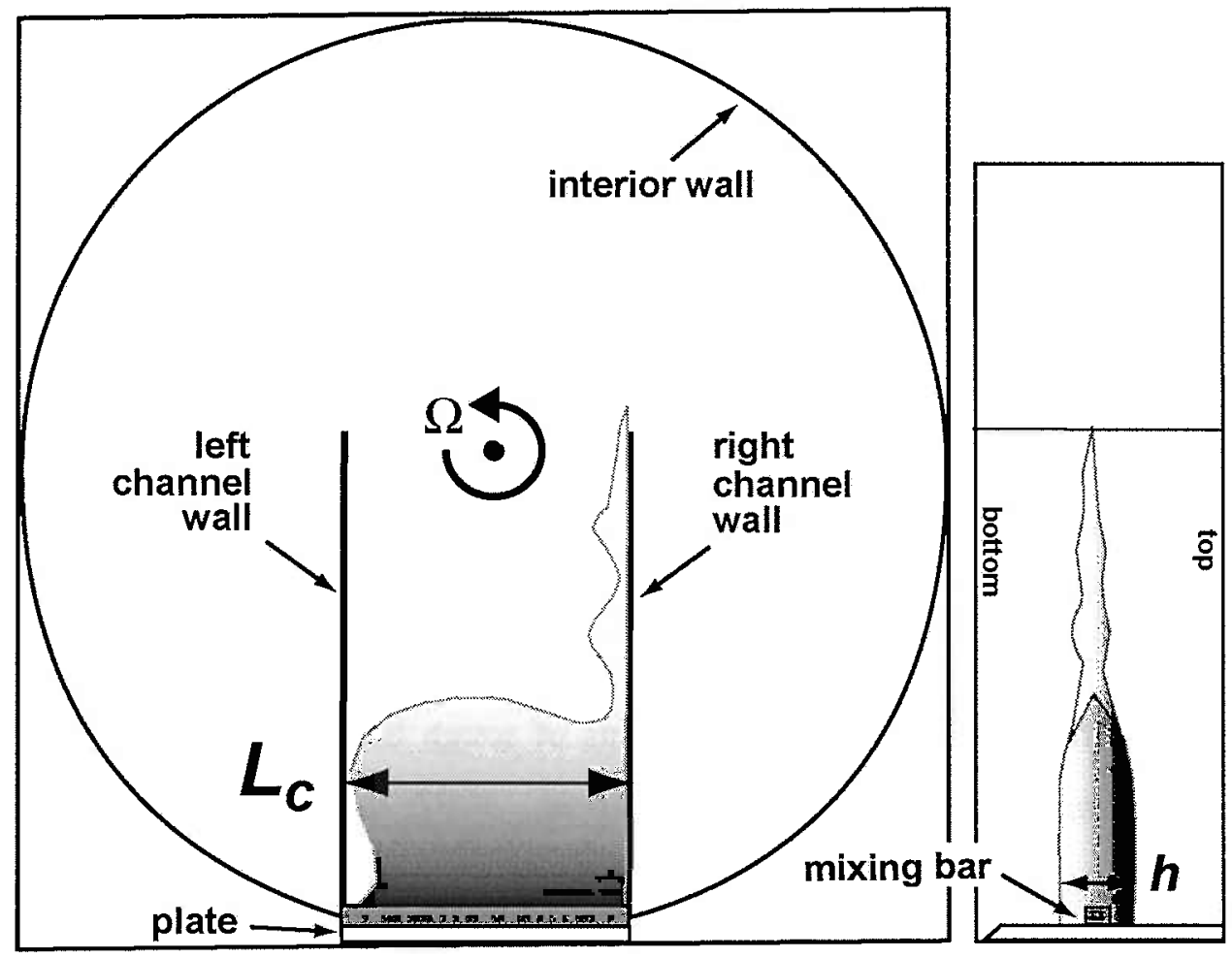

Figure 5.1: The experimental apparatus with a channel of width, $L_{C}$. Sketch showing a top view of the $114 \times 114 \mathrm{~cm}$ square glass tank, the circular interior wall, the channel walls, and the mixing bar and support plate. To the right of the tank is a $45^{\circ}$ mirror giving a side view of the bar. A region of mixed fluid is shown in front of the bar and a boundary current is shown along the right channel wall. Area measurements are made from the top view. The height $h$ is measured from the mirror view at the site of the doubleheaded arrow. 
In the previous experiments, features of vertical boundary mixing in an open basin are interpreted through a model of lateral entrainment (see Chapter 4.4 and Figure 4.16). For example, the restrictive effect of rotation on mixed fluid production, and the unexpected absence of boundary currents are both associated with a pattern of horizontal inflow at the sides of the mixing region. When channel walls confine the mixer and block the inflow, these features may change. The production of mixed fluid, depending on alternative avenues of horizontal or vertical entrainment, may be curtailed. The export of mixed fluid, given the immediate presence of the channel wall, may now occur as a boundary current. These possibilities are investigated in a series of channel mixing experiments, covering a range of values of $N$ and $f$.

The main set of experiments is conducted with a channel size $L_{C}$ corresponding to the maximum mixing bar length, $L_{C}=30.5 \mathrm{~cm}$. The bar frequency is fixed, $\omega=14 \mathrm{~s}^{-1}$. The experimental values for the buoyancy and Coriolis frequencies are $N=[0.3,0.6,0.9] \mathrm{s}^{-1}$, and $f=[0,0.1,0.2,0.3,0.6,0.9,1.2] \mathrm{s}^{-1}$, respectively. After the results of the rotating and nonrotating experiments are analyzed for $L_{C}=30.5 \mathrm{~cm}$, the role of channel width is investigated in experiments with $L_{C}=[25.4,20.3] \mathrm{cm}$.

Figure 5.2 is a regime diagram of the experiments. Each experiment is characterized by two nondimensional numbers, $N / f$ and $L_{C} / L_{R}$, where $L_{R}$ is the deformation radius defined by

$$
L_{R} \equiv \frac{N}{f} h_{m} .
$$

The vertical scale is the height of the mixed layer, $h_{m}$, an empirically determined function of the buoyancy height scale (3.13),

$$
H_{N} \equiv \gamma\left(\frac{\omega}{N}\right)^{1 / 2}
$$

where the constant of proportionality $\gamma=0.98 \mathrm{~cm} \mathrm{(3.12).} \mathrm{The} \mathrm{derivation} \mathrm{of} \gamma$ and the scale height can be found in Chapter 3. The determination of $h_{m}$ will be presented in 
section 5.3.a. The parameter $L_{C} / L_{R}$ compares the width of the channel to the distance within which the flow will feel the effects of rotation. The experiments cover the parameter ranges, $\frac{1}{4} \leq N / f \leq 9$ and $1 \leq L_{C} / L_{R} \leq 18$.

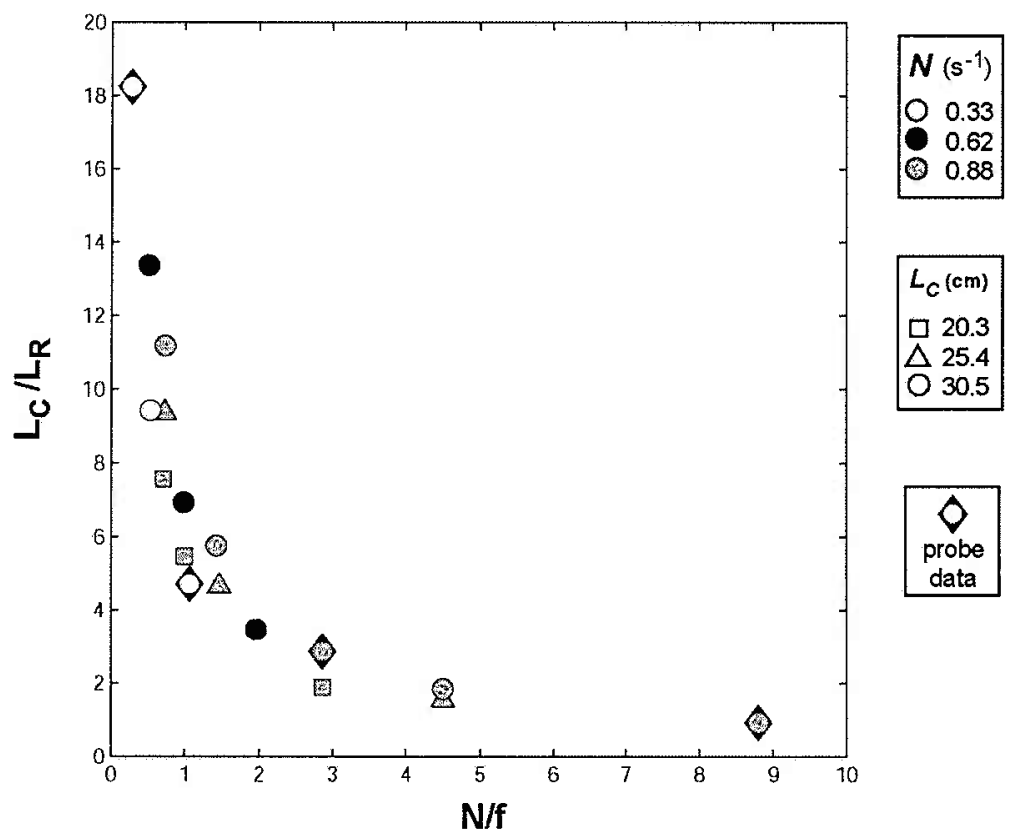

Figure 5.2: Regime diagram. Each experiment is shown with respect to $N / f$, the ratio of buoyancy and Coriolis frequencies, and $L_{C} / L_{R}$, the ratio of channel width to the deformation radius (5.1). The symbols indicate initial buoyancy frequency and channel width. The diamonds designate the probe experiments.

In the chapter on vertical boundary mixing, experiments are characterized by the nondimensional parameters, $N / f$ and $L_{f} / L_{B}$, where $L_{B}$ is the bar length and $L_{f}$ is the rotational length scale, defined in Chapter 3.5 by equation (3.14),

$$
L_{f} \equiv \gamma\left(\frac{\omega}{f}\right)^{1 / 2} .
$$


The parameter $L_{f} / L_{B}$ compares the length scale of rotationally controlled turbulence to the length of the turbulent zone. Recall that the lateral entrainment zone scaled with $L_{f}$ (see Figure 4.16). In the unconfined boundary mixing experiments the production of mixed fluid is found to be a function of $L_{f}$ and independent of $L_{B}$ over the range tested. In the channel experiments $L_{C}$ corresponds to $L_{B}$ and the same parameter range is covered, $0.1 \leq L_{f} / L_{C} \leq 0.4$.

Figure 5.1 sketches a typical distribution of mixed fluid with both a region of mixed fluid in front of the bar and a boundary current along the right hand channel wall. Neutrally buoyant dye is slowly injected at three sites just in front of the mixing bar and quickly disperses throughout the turbulent region. The dye moves with the mixed fluid as a marker, as discussed in Chapter 4.3.a, and measurements are made from digitized images of the dye. The overhead camera records a direct view of lateral spreading and the image of layer thickness is reflected in the $45^{\circ}$ side mirror. Precise information on the vertical density distribution is collected with a conductivity probe. The initial buoyancy frequency $N$ is recorded for each experiment prior to the initiation of mixing. Probe profiles are also used to evaluate horizontal density differences within the mixed layer. Cross channel transects are made through the center of the mixed region in four separate experiments which span the parameter range of the dye experiments with $N / f=\left[\frac{1}{4}, 1,3,9\right]$ and $L_{c} / L_{R}=[18,4.7,3,1]$. The duplicate experiments are indicated by diamonds on the regime diagram (Figure 5.2). Frequent transects are made over the course of the three-hour long experiments, and each transect consists of a series of closely spaced vertical density profiles. The data provide a picture of both the vertical and horizontal density distributions. The information is used in section 5.3.b to assess crosschannel variations in mixed layer height. In section 5.4, the horizontal density differences are used to calculate geostrophic velocities and infer circulation within the channel. 


\subsection{Qualitative description}

\section{2.a Evolution of the mixed layer}

The typical formation and spreading of mixed fluid is illustrated in Figure 5.3 with images from an experiment with $(\omega, N, f)=(14,0.61,0.62) \mathrm{s}^{-1}, \quad N / f=1$ and $L_{C} / L_{R}=7$. Turbulent mixing of the stratified fluid begins with the onset of the bar oscillation at $t=0$. Mixed fluid, generated at the bar, collapses into the interior, turns to the right and forms a boundary current along the right-hand wall. The bulge in the base of the boundary current in panel $a$ at $t=70 \mathrm{~s}$ is a developing cyclonic eddy. The eddy grows into a region of recirculating fluid in front of the bar (panel $b, t=360 \mathrm{~s}$ ). This cell expands to fill the width of the channel and spreads outward from the bar. In the experiment pictured here, a second, anticyclonic, eddy forms on the left-hand side of the channel (panel $d, t=900 \mathrm{~s}$ ). The bulk of mixed fluid is contained in the these cells, but the boundary current remains distinct and continues to advance. It follows the wall and turns the sharp corner at the end of the channel. The current develops regularly spaced, standing lobes (panel $b$ ) which elongate into the interior with time (panels $c$ and $d$ ). Panel $e$ at $t=1800$ s shows a lobe within the channel being engulfed by the cell of mixed fluid, and a lobe around the end of the channel wall stretching across the tank. By $t=3600 \mathrm{~s}$ (panel $f$ ), the latter lobe has established a separate pathway to the tank periphery. However, the majority of mixed fluid is still contained within the channel.

The two structures, the recirculating region and the boundary current, are also apparent in the side view of the mixed layer. Figure 5.4 shows images at $t=40 \mathrm{~s}$, when the boundary current is just emerging from the mixed region, and at $t=600 \mathrm{~s}$, when both structures are clearly defined. The recirculating zone is distinguished by its relatively uniform height, while the thinner boundary current tapers toward the nose. The dark patches on the boundary current are the laterally extended lobes. 

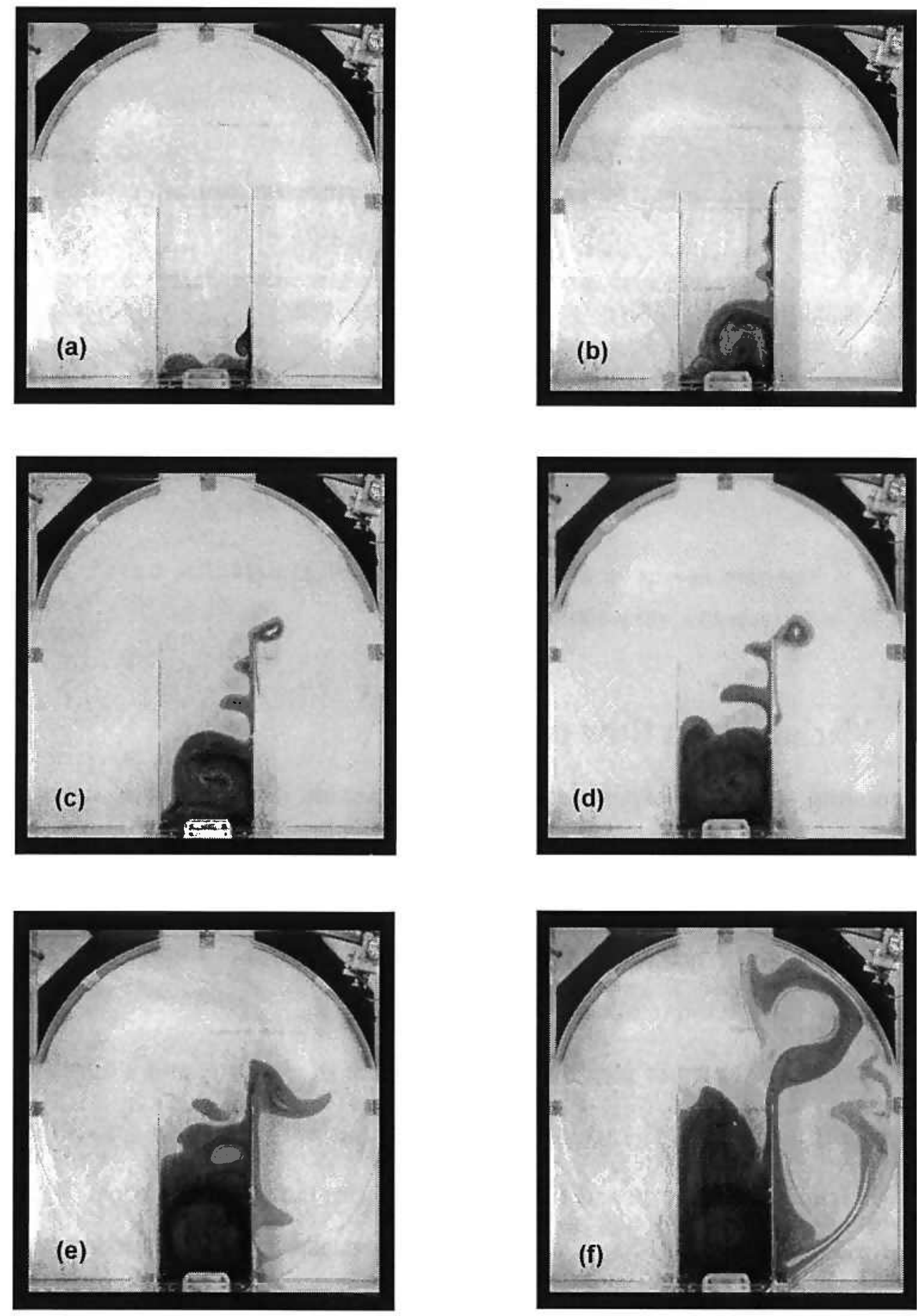

Figure 5.3: Dye images for an experiment with $(\omega, N, f)=(14,0.6,0.6) \mathrm{s}^{-1}, L_{c}=30.5 \mathrm{~cm}$, $N / f=1, L_{C} / L_{R}=7$. (a) $\mathrm{t}=70 \mathrm{~s}$, (b) $\mathrm{t}=360 \mathrm{~s}$, (c) $\mathrm{t}=600 \mathrm{~s}$, (d) $\mathrm{t}=900 \mathrm{~s}$, (e) $\mathrm{t}=1800 \mathrm{~s}$, (f) $\mathrm{t}=3600 \mathrm{~s}$. 

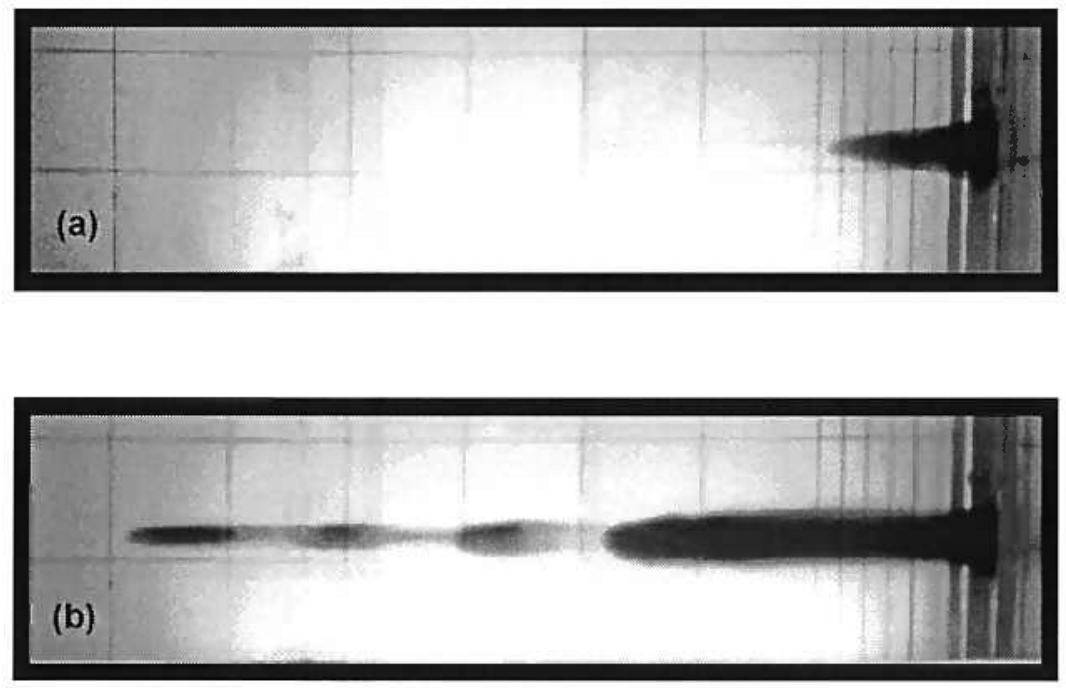

Figure 5.4: Side view images for an experiment with $(\omega, N, f)=(14,0.6,0.6) \mathrm{s}^{-1}, L_{c}=30.5 \mathrm{~cm}$, $N / f=1, L_{C} / L_{R}=7$. (a) $\mathrm{t}=40 \mathrm{~s}$, (b) $\mathrm{t}=600 \mathrm{~s}$.

\section{2.b Variations in flow patterns}

All the rotating, stratified experiments display the same dual structure, a recirculating region spanning the channel in front of the mixing bar, and a boundary current following the right-hand wall and exiting the channel. However, within this basic pattern there is considerable variety. Figure 5.5 shows images taken at $t=600 \mathrm{~s}$ during three experiments covering a range of values for $N / f$ and $L_{C} / L_{R}$.

The experiment shown in panel $a$ has a low value of $N / f=\frac{1}{2}$ and a correspondingly high value of $L_{C} / L_{R}=15$. The recirculation region has not yet spread across the channel and is still distinct from the mixed fluid emerging from the turbulent zone. The absence of dyed fluid along the left-hand wall suggests a possible avenue for entrainment of unmixed fluid into the turbulent zone. In panel $b$, where $N / f=2$ and $L_{C} / L_{R}=4$, a patch of undyed fluid can be seen to the left of the turbulent zone, but the fully developed recirculation cell now spans the channel width and extends halfway down its length. Panel $c$ shows the experiment with the highest ratio of stratification to rotation 
frequencies, with $N / f=9$ and $L_{C} / L_{R}=1$. The channel is nearly filled with mixed fluid and a wide flow exits the right-hand side of the channel. Only the darker dye in the interior of the channel suggests the presence of both a recirculating cell and boundary current. However, the two structures had been distinct until mixed fluid nearly filled the channel at $t \approx 500 \mathrm{~s}$.
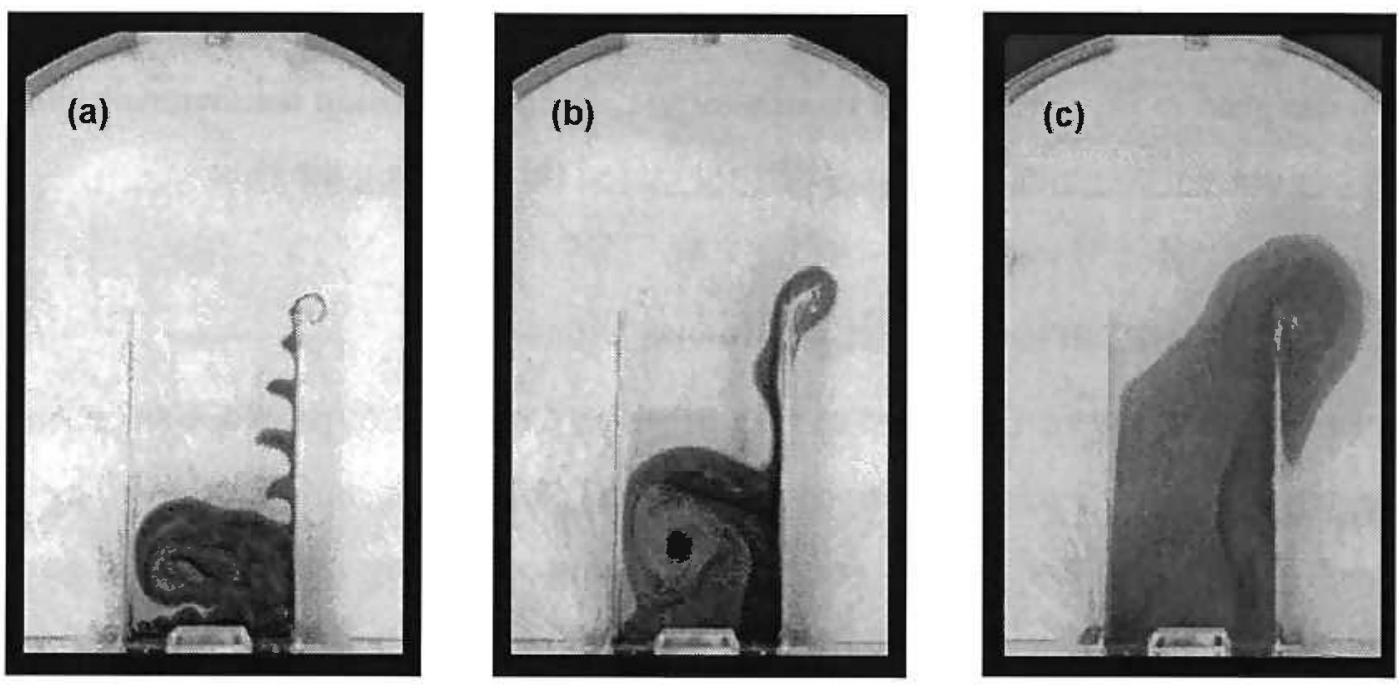

Figure 5.5: Dye images for three experiments with $\omega=14 \mathrm{~s}^{-1}$ and $L_{C}=30.5 \mathrm{~cm}$ at $\mathrm{t}=600 \mathrm{~s}$.
(a) $(N, f)=(0.6,1.2) \mathrm{s}^{-1}, \quad N / f=\frac{1}{2}, \quad L_{C} / L_{R}=15$
(b) $(N, f)=(0.6,0.3) \mathrm{s}^{-1}, \quad N / f=2, \quad L_{C} / L_{R}=3$,

(c) $(N, f)=(0.9,0.1) \mathrm{s}^{-1}, N / f=9, L_{c} / L_{R}=1$.

The images in panels $a$ and $b$ illustrate the changing character of the boundary current as $N / f$ increases and $L_{C} / L_{R}$ decreases. Panel $a$ shows a narrow current along the righthand wall characterized by multiple elongated lobes, while panel $b$ shows a somewhat broader current with just one rounded protrusion. Multiple disturbances appear on the edge of the boundary current in experiments where $L_{C} / L_{R}>5$. Figure $5.3 c$ shows the formation of two lobes in an experiment with $L_{C} / L_{R}=7$, and Figure $5.5 a$ shows four lobes on the boundary current of an experiment with $L_{C} / L_{R}=15$. The spacing between 
disturbances is roughly proportional to the deformation radius, $L_{R}$. The disturbances lengthen over time and when the amplitude exceeds five times the deformation radius they tend to break backward toward the mixing source and eventually merge with the recirculation region.

Baroclinic disturbances also develop along the rim of the recirculating cell in experiments where $L_{C} / L_{R}>9$. Both the rim and boundary current instabilities distribute mixed fluid further into the channel. However, the horizontal dispersion of mixed fluid within the channel does not directly affect the distribution of mixed fluid in the larger basin. As seen in Figure 5.3 $f$, the recirculating cells remain within the channel while the boundary current continues as the conduit for mixed fluid leaving the channel.

\section{2.c A comparison with previous work}

The laboratory study of Davies et al., described in Chapter 2.5, provides an interesting comparison to the present experiments. Their experimental configuration is a closed channel. They use a horizontally oriented, vertically oscillating meshed grid to generate turbulence at the narrow end of a rotating rectangular tank filled with linearly stratified salt water. The grid motion quickly establishes a mixed layer which collapses into the tank interior and forms a region of mixed fluid in front of the grid and a boundary current along the right-hand wall. The quantitative emphasis of the study is on the long term evolution of the mixed layer above and below the grid after the boundary current has returned to the source. However, Davies et al. also provide a qualitative picture of the horizontal distribution of the mixed fluid over the short time period before the boundary current reaches the end of the tank and begins its return, between five and thirty rotation periods after the onset of grid mixing. Visualization of the flow is achieved through marking the mixed fluid with an electrolytically precipitated tracer, and through streak photographs of neutrally buoyant particles. They show examples from two flow regimes: one with $N / f=1.2$ and $L_{C} / L_{R}=10$, and the other with $N / f=4.8$ and $L_{C} / L_{R}=2.5$. In the former, the tracer shows a protrusion at the base of the boundary current that grows 
into a cyclonic eddy and merges with the mixed fluid in front of the grid. In the latter, the tracer simply shows the region of mixed fluid spreading out from the grid, while the streak photography provides evidence of cyclonic circulation within the fluid. The qualitative pictures also show the boundary current as a distinct structure, with a propagation speed that scales with gravity current velocity and a width of the order of the deformation radius. Overall, the early distribution of mixed fluid in the closed container corresponds to the developing flows observed in the open channel.

\subsection{Quantitative analysis and scaling}

In the present experiments, the quantitative analysis considers the extent of vertical mixing, the volume production of mixed fluid, and its partition between the two flow structures, the region of recirculation and the boundary current. Vertical mixing is evaluated from measurements of the mixed layer height near the turbulent zone, but variations in layer height in the interior of the channel are treated separately for the recirculating cell and the boundary current. The volume of the recirculation region is calculated from area and height data collected from the time of its formation up to the development of instabilities or secondary cells. Data on boundary current length, height and area cover the period from the current's emergence to its arrival at the end of the channel.

\section{3.a Height of the mixed layer}

The height of the mixed layer is measured approximately four $\mathrm{cm}$ from the bar at the location marked by the small doubleheaded arrow in Figure 5.1. Measurements are taken until dye in the larger basin blocks the side view. Turbulent overturning at the bar quickly establishes a mixed layer height that remains nearly steady throughout the experiments. A mean height $h_{m}$ for each experiment is calculated from all measurements taken for $t \geq 300 \mathrm{~s}$. Figure 5.6 plots the mean height versus the buoyancy scale height, 
$\gamma\left(\frac{\omega}{N}\right)^{1 / 2}$ (5.2), for fourteen experiments with $L_{C}=30.5 \mathrm{~cm}$ and $\omega=14 \mathrm{~s}^{-1}$. The errorbars give the standard deviation about the mean height for the individual experiment. Only the larger values are visible. On average, the standard deviation is $5 \%$ of the mean value and the errorbar is smaller than the symbol size. A straight line fit with a zero intercept gives,

$$
h_{m}=(0.93 \pm 0.06) \gamma\left(\frac{\omega}{N}\right)^{1 / 2},
$$

where the constant, $\gamma=0.98 \mathrm{~cm}(3.12)$, is associated with the mixing mechanics, and $\omega$ and $N$ are the bar and buoyancy frequencies, respectively. The layer height is proportional to the relative strength of the imposed mixing and the ambient stratification, and is independent of rotation. The functional relationship is the same as that established for the vertical boundary experiments in Chapter 4, where layer height is given by (4.2), $h_{m}=(0.97 \pm 0.05) \gamma\left(\frac{\omega}{N}\right)^{1 / 2}$. The constants of proportionality for $h_{m}$ are nearly identical for unconfined and laterally confined mixing.

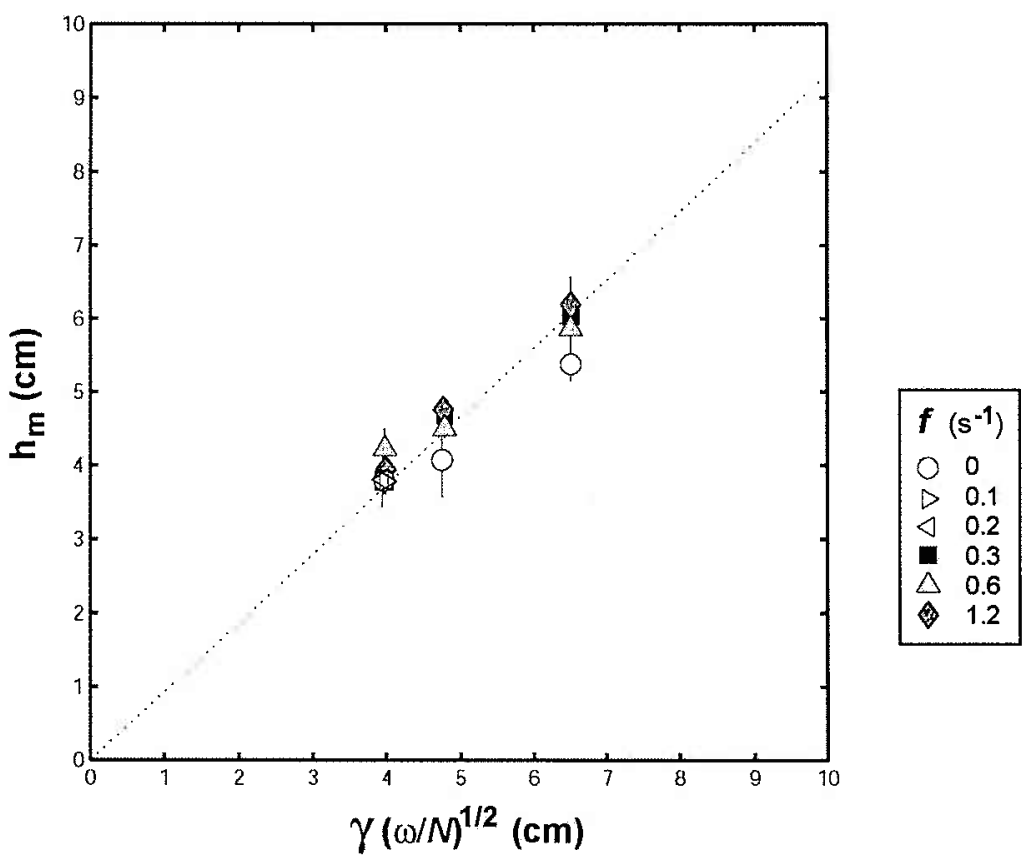

Figure 5.6: Mean height of mixed layer versus buoyancy scale height (5.2), for experiments with $\omega=14 \mathrm{~s}^{-1}, 0.3 \leq N \leq 0.9 \mathrm{~s}^{-1}, 0 \leq f \leq 1.2 \mathrm{~s}^{-1}$, and $L_{C}=30.5 \mathrm{~cm}$. Symbols indicate rotation rates for individual experiments. 
In the Davies et al. (1991) closed channel experiments, the mixed layer established an initial equilibrium thickness that scaled with $(K / N)^{1 / 2}$, where $K$ is a parameter which incorporates the mixing characteristics of their grid (see Chapter 2.2 and equation (2.5)). However, after the boundary current followed the tank perimeter back to the mixing region, Davies et al. report a resumption of vertical growth which they associate with the injection of mixed fluid from the boundary current. This development is not observed in the present experiments where the boundary current exits the channel and does not return to the source. The mixed layer maintains a steady state height throughout the experiments and is unaffected by the recirculation of mixed fluid in front of the bar.

\section{3.b Recirculation region}

The volume of fluid in the recirculation region is calculated from measurements of the mixed layer height and the horizontal area of dyed fluid in front of the mixing bar. As the mixed layer leaves the turbulent zone and adjusts to the ambient rotation and stratification it may develop variations in height. The recirculation region, seen from the side in Figure $5.4 b$, has a well-defined, relatively uniform height, but the side view, a horizontally integrated image of dyed fluid, shows only the maximum height. A picture of height variability through the approximate center of the recirculation region is obtained from conductivity probe measurements taken along cross channel transects $17 \mathrm{~cm}$ from the mixing bar. The cross sections of layer thickness inferred from the probe data are useful for identifying flows within the channel (see section 5.4), as well as for calculating mixed fluid volume within the recirculation region.

Figure 5.7 shows data from a transect of the channel taken at $t \approx 50$ min during an experiment with $(\omega, N, f)=(14,0.33,0.31) \mathrm{s}^{-1}$ and $L_{C} / L_{R}=4.7$. The section is comprised of seven vertical density profiles. In the plot the profiles are staggered according to their cross channel position from left to right, looking outward from the mixer. The profile spacing is closer on the right-hand side at the expected site of the boundary current. Each profile shows a homogeneous layer located at middepth and 
separated from the linearly stratified fluid above and below it by sharp density interfaces. Together the profiles indicate a small but definite thinning of the mixed layer toward the center of the channel, consistent with a cyclonic recirculation.

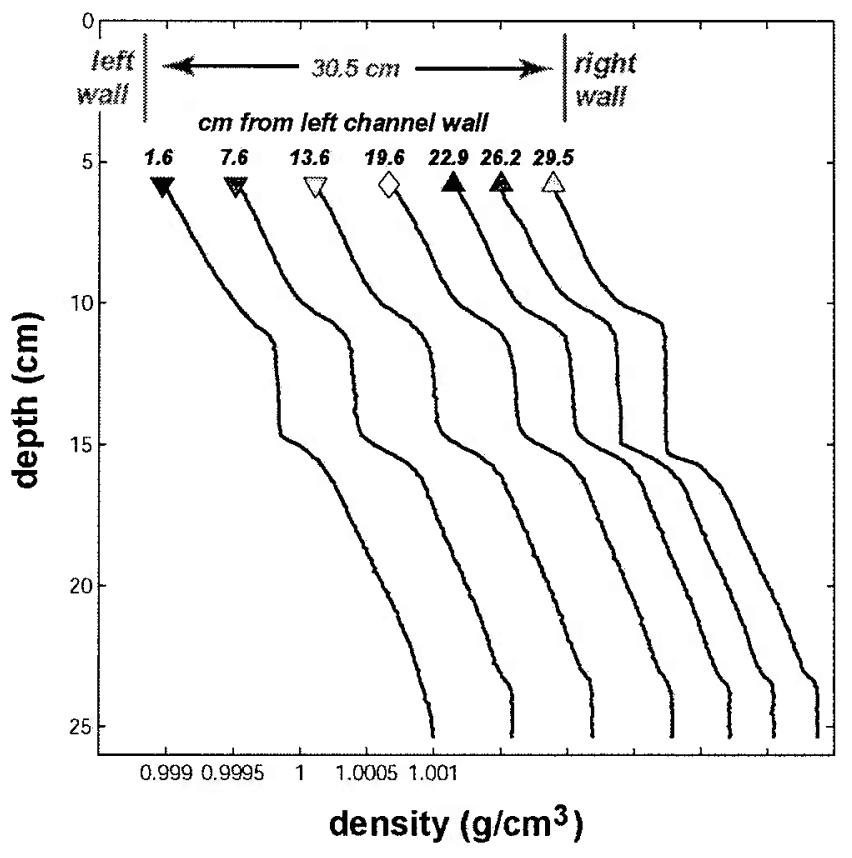

Figure 5.7: Density profiles for experiment with $(\omega, N, f)=(14,0.3,0.3) \mathrm{s}^{-1}$ and $L_{C}=30.5 \mathrm{~cm}$, taken 17 $\mathrm{cm}$ in front of the mixing bar at $\mathrm{t}=50 \mathrm{~min} . \quad N / f=1, L_{C} / L_{R}=4.7$. Profiles are staggered according to their spacing across the channel. The left-hand profile is pegged to the correct density.

The probe data is analyzed in the expectation that the height of the mixed layer will conform to some regular geometry related to the background rotation and stratification, and thus provide a formula for estimating height variations in the dye experiments. To arrive at a formula, the recirculating flow is modelled as a cylinder of height $h_{m}$ with antisymmetric cone-shaped upper and lower surfaces. Once the density profiles indicate that mixed fluid has filled the channel, the slope of the cone is calculated for each transect from measurements of the maximum and mean heights of the homogeneous layer. A 
characteristic slope, $\Delta h /\left(\frac{1}{2} L_{C}\right)$, is then defined for each experiment from the full set of transects.

Ideally a flow in geostrophic balance would show a change in height, $\Delta h \sim(f / N) l$, where $l$ is the radius of the recirculating region. Instead, the data, which cover the range $\frac{1}{4} \leq N / f \leq 9$, show $\Delta h \sim(f / N)^{1 / 2} l$, where $l=\frac{1}{2} L_{C}$. This result is incorporated in a height adjustment for the volume calculations,

$$
h_{*}=h_{m}-0.03\left(\frac{f}{N}\right)^{1 / 2} l(t) .
$$

Here, $h_{m}$ is the mean mixed layer height measured near the turbulent zone for the individual experiment. The second term is $\Delta h$, as a function of $l(t)$, the half-length of the recirculation region measured parallel to the mixing bar. $\Delta h$ is weighted by a factor which incorporates the volumetric effects of the assumed cone geometry. Caution is needed in using $h_{*}$, since equation (5.5) is derived from a highly simplified geometry and limited experimental data. In the discussion which follows, volume calculations based on the adjusted height, $h_{*}$, are compared to those using the unadjusted mean height, $h_{m}$.

Area measurements of the horizontal spreading of dyed fluid in front of the bar are shown in Figure $5.8 a$, which plots dimensional area versus time. Figure $5.8 b$ plots the normalized data, with area scaled by $L_{C}\left(L_{R} L_{f}\right)^{1 / 2}$ and time scaled by $f^{-1}$. The term $\left(L_{R} L_{f}\right)^{1 / 2}$ is the square root of the deformation radius $L_{R}$ (5.1) and the rotational length scale $L_{f}(5.3)$. The channel width, $L_{C}$, a constant in these experiments, is included in the scaling for dimensional reasons. Its role is investigated separately in the experiments discussed in section 5.3.d. The area grows approximately as $t^{1 / 2}$, with $A_{m} \propto t^{0.59 \pm 0.14}$. In Figure $5.8 b$ the normalized area measurements are shown superimposed on the $(f t)^{1 / 2}$ curve. The data collapse quite well to

$$
A_{m}=(0.32 \pm 0.04) L_{C}\left(L_{R} L_{f}\right)^{1 / 2}(f t)^{1 / 2} .
$$


In the vertical boundary mixing experiments of Chapter 4, area growth is described as a function of $L_{R} L_{f} f t$, (see equation (4.6), (4.7) and Figure 4.13). Interestingly, the square root of these terms appears in the scaling of mixing in a channel, and the rate of area growth, which was constant in the unconfined case, decreases with time in the confined case.
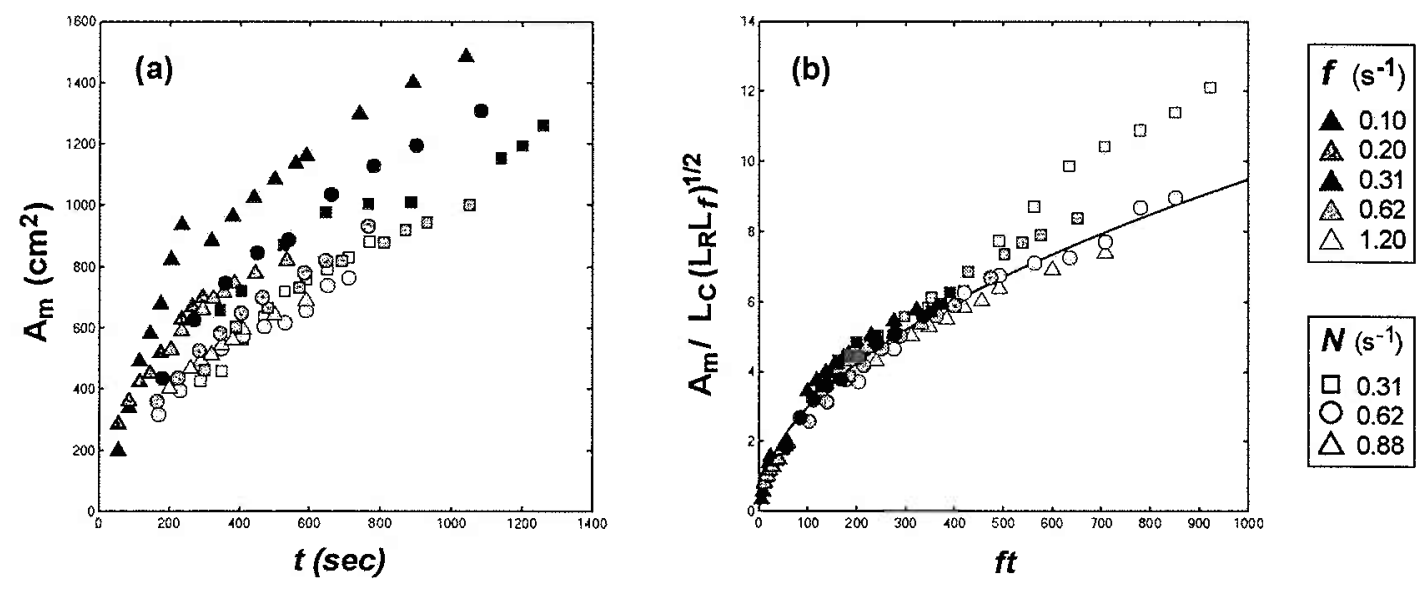

Figure 5.8: Horizontal area of mixed fluid for all rotating, stratified experiments with $L_{C}=30.5 \mathrm{~cm}$. Symbols indicate values of $N$ and $f$. (a) Area $\left(\mathrm{cm}^{2}\right)$ vs time (sec), (b) Area normalized by $L_{C}\left(L_{R} L_{f}\right)^{1 / 2}$ vs nondimensional time $f t . L_{R}$ and $L_{f}$ are given by (5.1) and (5.3), respectively.

The gradual blockage of the channel by mixed fluid offers a possible explanation for the observed scaling. If mixed layer growth and lateral spreading initially follow the pattern of the unconfined experiments, the recirculating cell will expand with a radius, $r \sim\left(L_{R} L_{f} f t\right)^{1 / 2}$. As it grows, it will squeeze shut the pathway along the left wall which supplies ambient fluid to the mixing zone. This should scale as

$$
\frac{d}{d t}\left|L_{C}-2 r\right| \sim\left(L_{R} L_{f}\right)^{1 / 2} f^{1 / 2} t^{-1 / 2} .
$$

The lateral entrainment of unmixed fluid will thus decrease as $t^{-1 / 2}$. 
Volume is calculated from the product of area and height, $A(t) \times h_{*}(t),(5.5)$. The effect of using the adjusted height factor $h_{*}$ instead of $h_{m}$ is a general reduction in calculated volumes. The reductions range from $3 \%$ to $11 \%$ of volumes calculated from the formula, $A(t) \times h_{m}$, and are greatest in those experiments with the lowest values of $N / f$. The reductions also tend to increase over time as more of the mixed fluid in front of the bar is involved in recirculation (compare, for example, panels $b$ and $c$ in Figure 5.3). However, the volume, based on $h_{*}$, scales the same as volume based on the mean heights for each experiment. Figure $5.9 a$ plots the normalized volume versus $f t$. Figure $5.9 b$ presents the data in a $\log \log$ plot illustrating the $t^{1 / 2}$ behavior. The data give

$$
V_{m}=(0.30 \pm 0.04) L_{C}\left(L_{R} L_{f}\right)^{1 / 2} h_{m}(f t)^{1 / 2} .
$$

Here, the scale factor $h_{m}$ denotes the empirical relation for height (5.4).

The equation for volume growth (5.8) is derived from the best fit of the full data set to variations in $N$ and $f$, but data points from two experiments, one at each end of the $N / f$ parameter range, appear as outliers in Figure 5.9. The light gray squares, above the upper right segment of the data curves, indicate exceptionally rapid and sustained production of mixed fluid in the experiment with $N / f=\frac{1}{4}$ and $L_{C} / L_{R}=18$. The black triangles at the lower left of the loglog plot, Figure $5.9 b$, show low initial volumes and a high growth rate in the experiment with $N / f=9$ and $L_{C} / L_{R}=1$. The latter experiment is pictured in Figure $5.5 \mathrm{c}$ at $t=600 \mathrm{~s}$. The observed broadening of the boundary current and the volume measurements of the recirculation region for $N / f=9$ may represent a change in mixing dynamics. However, the rapid filling of the channel and the low rotation rate, $f=0.1 \mathrm{~s}^{-1}$, restrict data collection to less than four rotation periods, and there is little time for mixed fluid flow to adjust to the background rotation. In the former experiment, where $N / f=\frac{1}{4}$, the high volume of the recirculation region may simply be indicative of experimental variability. In the probe experiment with identical parameters, the density profiles do not show a similar spreading of the mixed layer. 

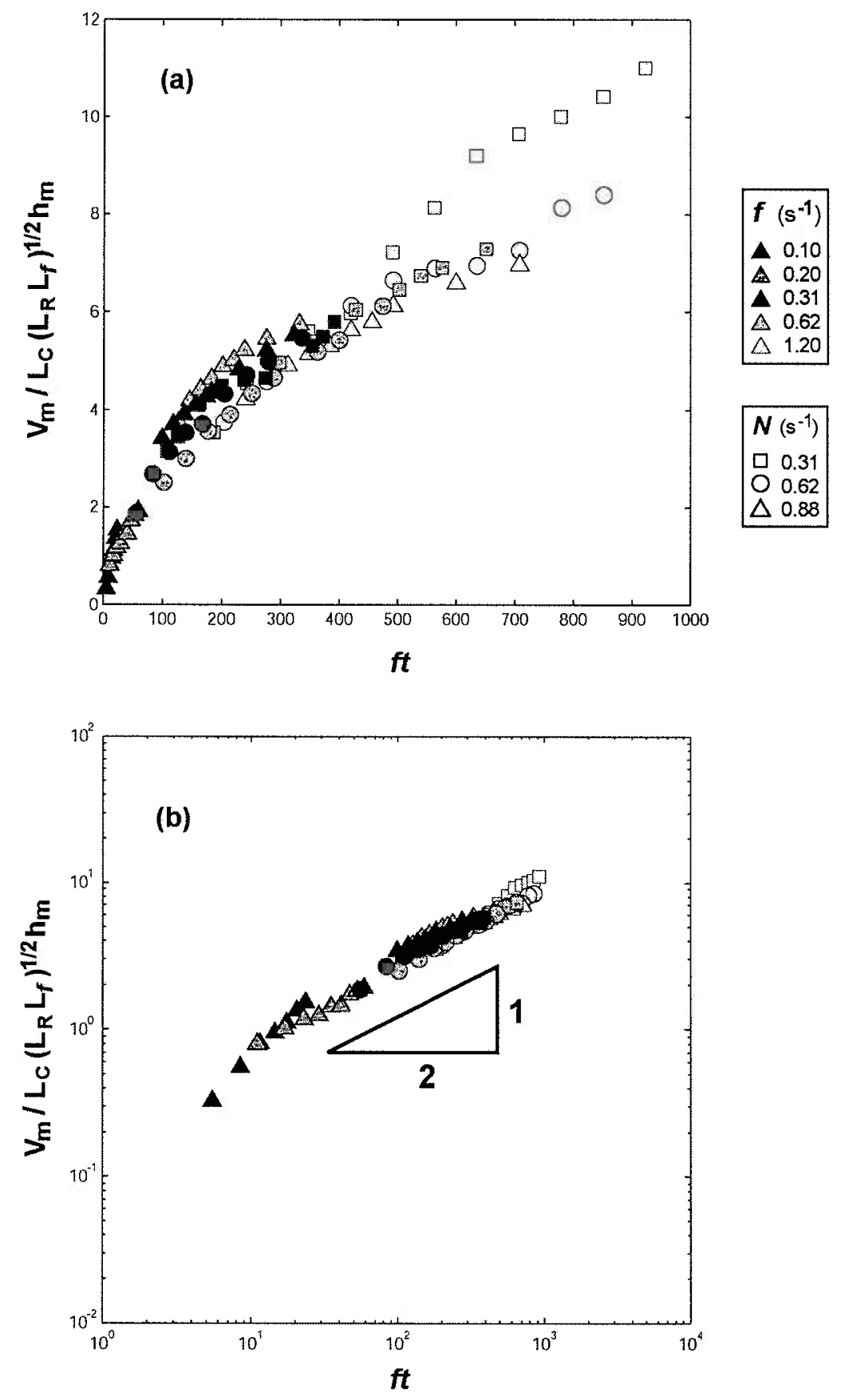

Figure 5.9: Volume of mixed fluid for all rotating, stratified experiments with $L_{C}=30.5 \mathrm{~cm}$. Symbols indicate values of $N$ and $f$. (a) Volume normalized by $L_{C}\left(L_{R} L_{f}\right)^{1 / 2} h_{m}$ vs nondimensional time $f t$. $L_{R}, L_{f}$, and $h_{m}$ are given by (5.1), (5.3) and (5.4), respectively. (b) The same data in a loglog plot. 


\section{3.c Boundary current}

It is necessary to account for the total production of mixed fluid in order to understand mixing in the laterally confined setting. The qualitative picture has emphasized the body of mixed fluid in front of the turbulent zone, but the boundary current also carries a portion of the mixed fluid. Moreover, the boundary current is the conduit for mixed fluid exiting the channel into the basin interior. This section estimates the volume of the boundary current and examines its propagation along the channel wall.
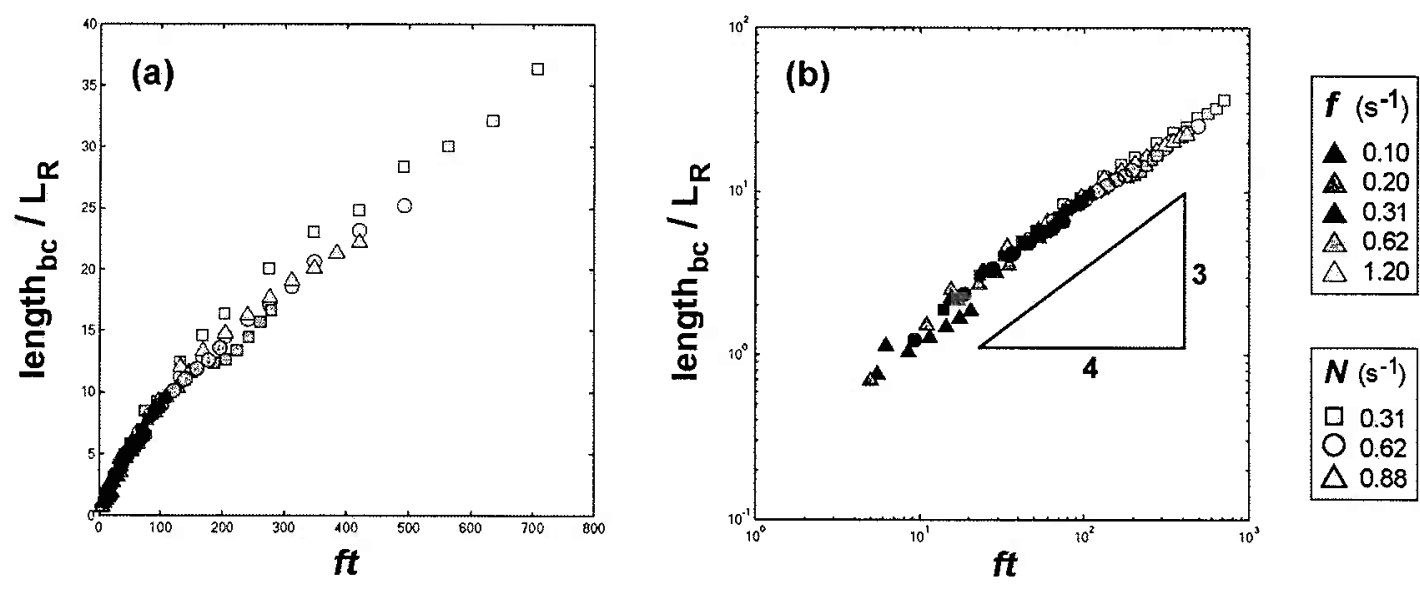

Figure 5.10: Advance of the boundary current for all rotating, stratified experiments with $L_{C}=30.5 \mathrm{~cm}$. Symbols indicate values of $N$ and $f$. (a) Length normalized by $L_{R}(5.1)$ vs nondimensional time $f t$. (b) The same data in a loglog plot.

The advance of the boundary current is measured by the position of the dyed fluid in the side view images, from the onset of mixing until the current leaves the channel. Figure $5.10 a$ plots the length of the boundary current normalized by the deformation radius, $L_{R}(5.1)$, versus nondimensional time, $f t$. Figure $5.10 \mathrm{~b}$ displays the same data in a $\log \log$ plot. The boundary current advances approximately as $t^{3 / 4}$,

$$
l_{b c}=(0.25 \pm 0.03) L_{R}(f t)^{0.76 \pm 0.14} .
$$

The rate of advance is proportional to $N h_{m}$, the speed of a gravity current with height $h_{m}$, 


$$
\frac{d l_{b c}}{d t}=0.19 N h_{m}(f t)^{-0.24} .
$$

The slowing of the current nose may be attributable to mixed fluid entering the growing lateral lobes. The boundary current area, $A_{b c}$, measured from the top plan view, shows a linear growth.

A rough estimate of the volume of mixed fluid contained in the boundary current is obtained from a simple formula: $V_{b c}=\frac{4}{9} h_{b c} \times A_{b c}$, where $h_{b c}$ is the height of the boundary current measured where it emerges from the recirculating region (see Figure $5.4 b$ ). The constant $\frac{4}{9}$ follows from an assumption that the vertical distribution of mixed fluid approximates a parabolic shape as it moves out parallel to the wall and as it spreads laterally into the channel. The height $h_{b c}$ remains relatively constant, thus giving a linear growth in boundary current volume.

As the volume growth of the recirculation region decays with time, the boundary current carries an increasing percentage of the total mixed fluid. During the time period of the measurements, these percentages remain small. For $N / f \leq 3$, the maximums range from $1 \%$ to $4 \%$ of the total mixed fluid volume, increasing with increasing values of $N / f$. The experiments with $N / f=4.5$ and $N / f=9$ show higher maximums, $13 \%$ and $14 \%$, respectively. However, the $N / f=9$ data points, which appear as outliers on the volume plot in Figure 5.9, remain outliers even with the addition of boundary current measurements. Overall, the boundary current volumes do not substantially change the previously determined relation for volume growth (5.8).

\section{3.d Variations in channel width}

In the unconfined experiments the production of mixed fluid was found to be independent of the horizontal extent of the mixing region given by the bar length, $L_{B}$. In the confined experiments the mixing region length, given by the channel width, $L_{C}$, is included in the scaling of mixed fluid volume for dimensional reasons, even though it has remained a constant $30.5 \mathrm{~cm}$. The validity of the scaling is examined here in two additional sets of 
experiments with $L_{c}=[20.3,25.4] \mathrm{cm}, \frac{2}{3}$ and $\frac{5}{6}$ of the original width, respectively. For each channel width, three experiments are conducted with a similar buoyancy frequency, $N \approx 0.9 \mathrm{~s}^{-1}$, and different values of the Coriolis frequency, such that $0.7 \leq N / f \leq 4.5$ and $9.5 \geq L_{C} / L_{R} \geq 1.5$. The experiments are marked on the regime diagram, Figure 5.2 , by the squares $\left(L_{C}=20.3 \mathrm{~cm}\right)$ and triangles $\left(L_{C}=25.4 \mathrm{~cm}\right)$. The bar frequency $\omega=14 \mathrm{~s}^{-1}$.

As in the base set of channel experiments, the height of the mixed layer is measured four $\mathrm{cm}$ in front of the bar. Figure 5.11 shows the mean heights versus the buoyancy height scale (5.2). The new data is superimposed on the first data set and corresponds to the original determination of height, $h_{m}=(0.93 \pm 0.06) \gamma\left(\frac{\omega}{N}\right)^{1 / 2} \mathrm{~cm}$. As in the unconfined case, the height of the mixed layer is unaffected by the length of the mixing region.

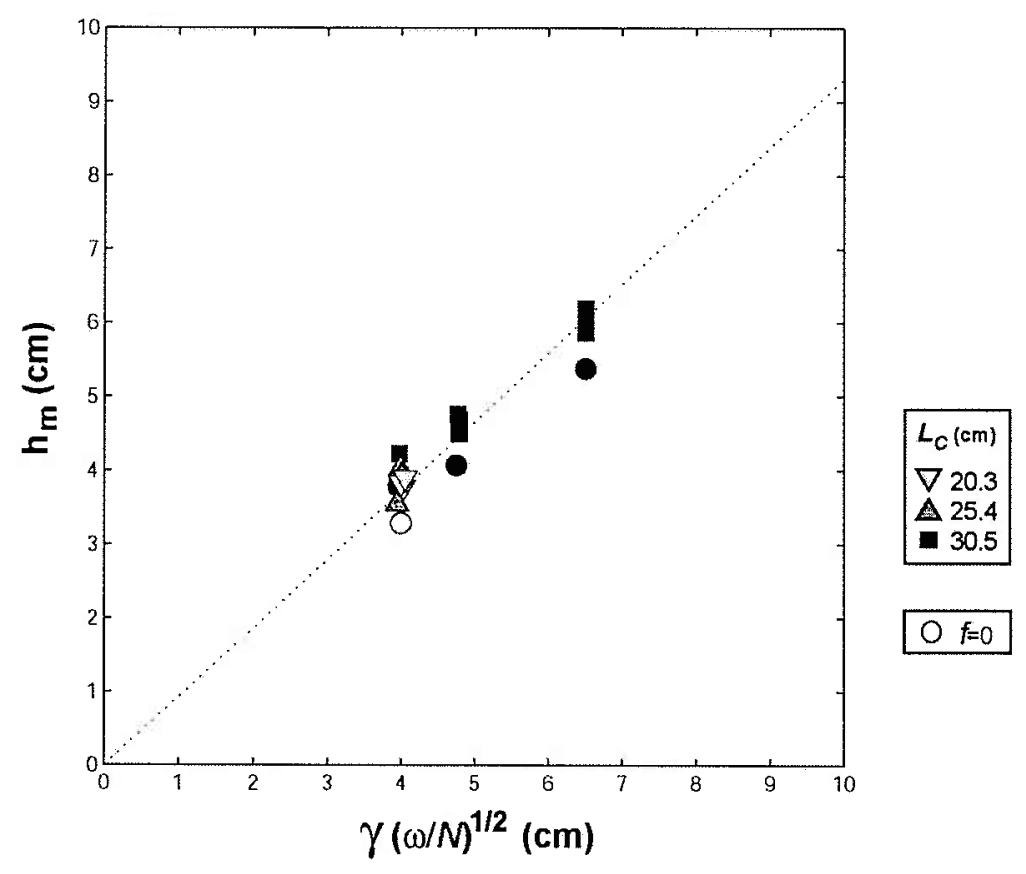

Figure 5.11: Mean height of mixed layer versus buoyancy scale height (5.2), for experiments with all channel widths. The black symbols indicate the original set with $L_{C}=30.5 \mathrm{~cm}$ (see Figure 5.6). Mean heights of the nonrotating experiments (all channel widths) are indicated by circles. 
Volume calculations for the mixed fluid in the recirculation region are presented in Figure 5.12. For a fixed channel width the best fit of volume growth to the external parameters, $N$ and $f$, is achieved using the scale factors, $L_{C}\left(L_{R} L_{f}\right)^{1 / 2} h_{m}$, from equation (5.8), derived for volume growth in the original channel experiments. Panel $a$ shows the volume data from all three sets, normalized by these scale factors and a single constant $(30.5 \mathrm{~cm})$, versus nondimensional time, $f t$. In panel $b$ the volume data are scaled by the appropriate channel width, $L_{C}=[20.3,25.4$, or 30.5$] \mathrm{cm}$. The experiments conducted in the $\frac{5}{6}$ channel are on the low side, and one experiment, with $N / f=4.5$, is anomalously low. Nevertheless, the overall collapse of the data indicates that the volume of mixed fluid in the recirculation region is directly dependent on channel width.
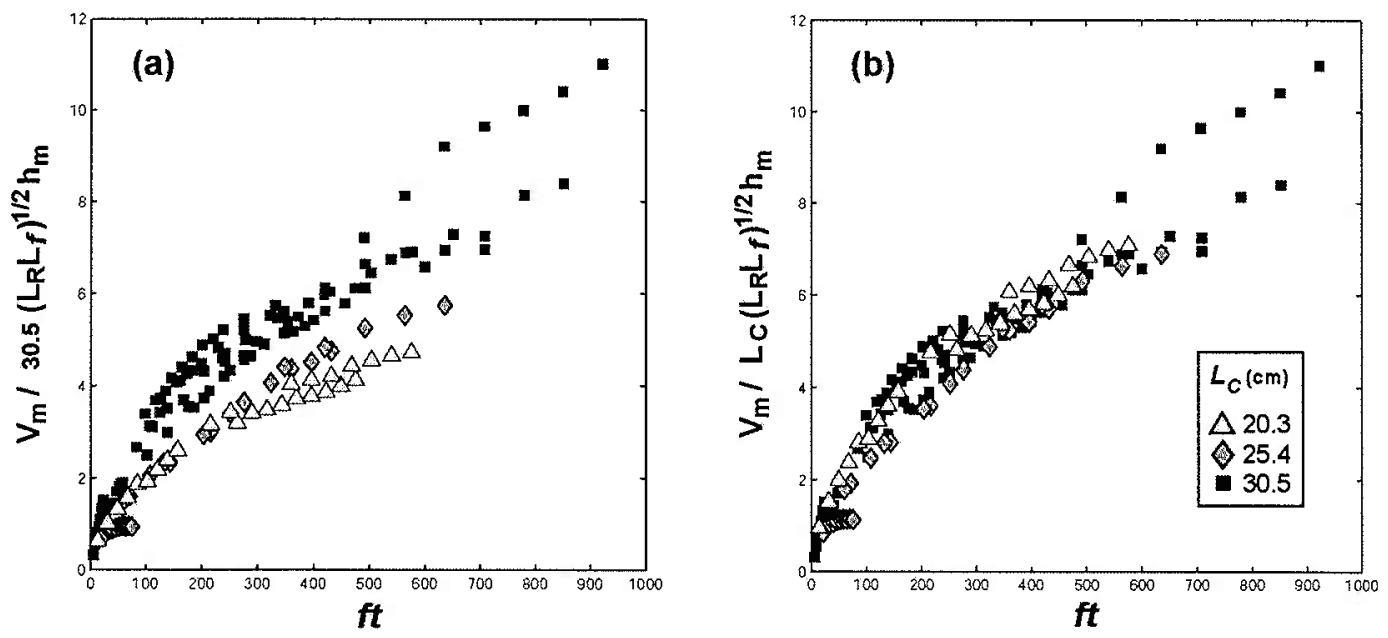

Figure 5.12: $\quad$ Volume of mixed fluid for all channel widths. The small squares indicate the original set with $L_{C}=30.5 \mathrm{~cm}$ (see Figure 5.9). (a) Volume normalized by a constant times $\left(L_{R} L_{f}\right)^{1 / 2} h_{m}$ vs $f t$, with $L_{R}, L_{f}$, and $h_{m}$ given by (5.1), (5.3) and (5.4), respectively. (b) Volume normalized by the appropriate channel width, $L_{C}$, times $\left(L_{R} L_{f}\right)^{1 / 2} h_{m}$ vs $f t$

The boundary currents are not noticeably dependent on channel width within the range investigated. Figure 5.13 is a loglog plot of boundary current length, normalized by the deformation radius (5.1), versus $f t$. The boundary current advances as $l_{b c} \sim t^{3 / 4}$, 
propagating with a speed proportional to $N h_{m}$. The data points from the experiments with $L_{C}=[20.5,25.4] \mathrm{cm}$ fall slightly below the original $L_{C}=30.5$ data, but not in an order related to relative channel width.

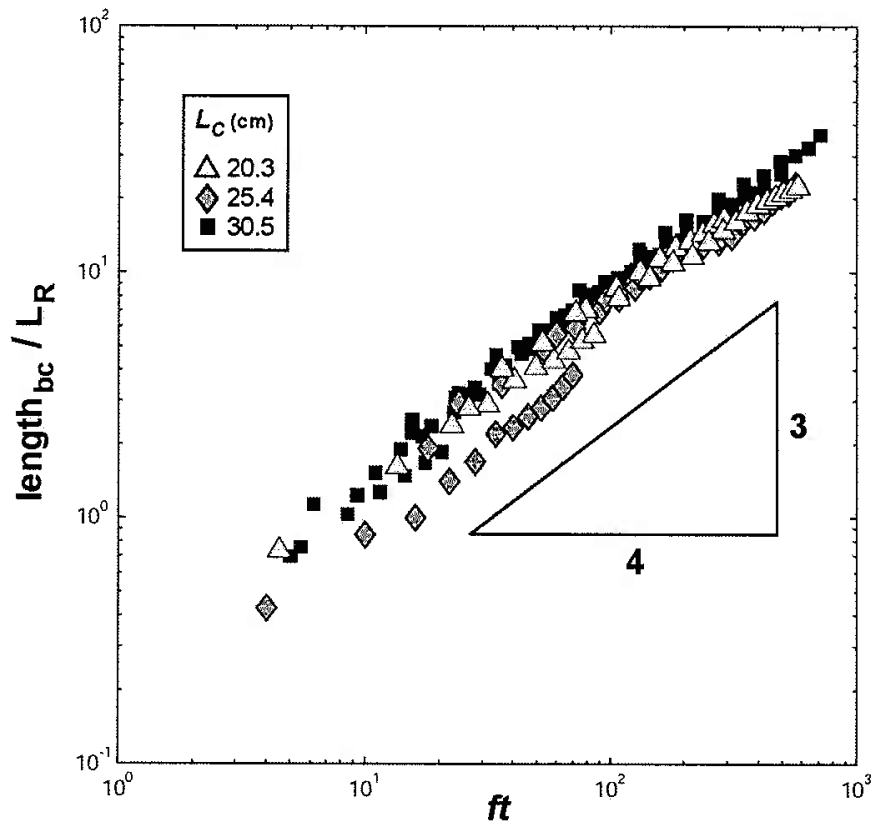

Figure 5.13: Advance of the boundary current for all channel widths. The small squares indicate the original set with $L_{C}=30.5 \mathrm{~cm}$ (see Figure 5.10). Length normalized by $L_{R}(5.1)$ vs nondimensional time $f t$.

The partition of mixed fluid between the recirculation region and the boundary current also shows no significant change. The percentage of the total mixed fluid carried by the boundary current increases with time, but remains small during the measurement period and appears to be a function of $N / f$ rather than $L_{C} / L_{R}$. This relationship is illustrated by a comparison between an experiment in the $20.3 \mathrm{~cm}$ channel with $\left(N / f, L_{C} / L_{R}\right)=(3,2)$ and one in the $30.5 \mathrm{~cm}$ channel with $\left(N / f, L_{C} / L_{R}\right)=(4.5,2)$. Although both experiments have the same value of $L_{C} / L_{R}$, the boundary current in the first experiment carries less than $2 \%$ of the total volume of mixed fluid, while in the second case it carries approximately $13 \%$. In the anomalously low volume experiment, 
with $\left(N / f, L_{C} / L_{R}\right)=(4.5,1.5)$, the boundary current contains $15 \%$ of the mixed fluid, comparable to the $13 \%$ cited above. In all other experiments with $L_{C}=[20.3,25.4] \mathrm{cm}$, $N / f \leq 3$ and the boundary current carries $1 \%$ to $4 \%$ of the total volume.

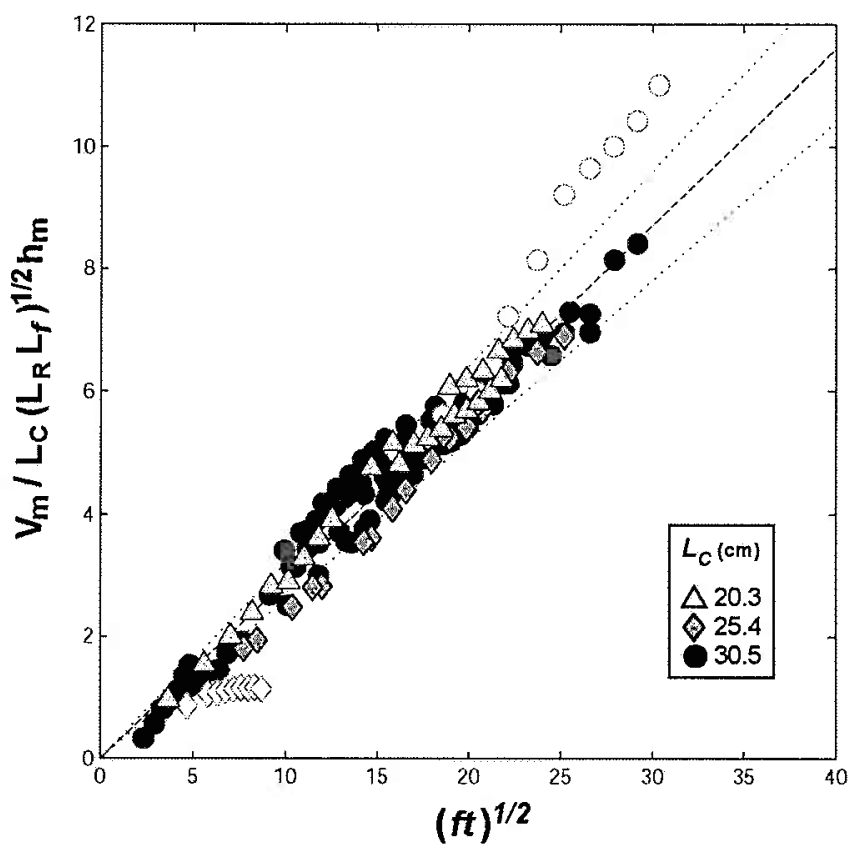

Figure 5.14: Volume of mixed fluid for all experiments. The symbols indicate the channel width, $L_{C}$. Volume normalized by $L_{C}\left(L_{R} L_{f}\right)^{1 / 2} h_{m}$ vs the square root of nondimensional time $(f t)^{1 / 2} . L_{R}, L_{f}$, and $h_{m}$ are given by (5.1), (5.3) and (5.4), respectively.

Figure 5.14 plots the volume of mixed fluid in the recirculation region for all three channel sizes. The volume is normalized by $L_{C}\left(L_{R} L_{f}\right)^{1 / 2} h_{m}$, and is now plotted versus nondimensional time to the one-half power, $(f t)^{1 / 2}$. The full data set gives $V_{m}=(0.29 \pm 0.05) L_{C} h_{m}\left(L_{R} L_{f}\right)^{1 / 2}(f t)^{1 / 2}$ with a correlation coefficient, $r=0.97$. When the two anomalous experiments are excluded, that is, the experiment with $L_{C}=30.5 \mathrm{~cm}$ and $N / f=\frac{1}{4}$, and the one with $L_{C}=25.4 \mathrm{~cm}$ and $N / f=4.5$, the combined data give, 


$$
V_{m}=(0.29 \pm 0.03) L_{C} h_{m}\left(L_{R} L_{f}\right)^{1 / 2}(f t)^{1 / 2} .
$$

The correlation coefficient for this fit is $r=0.98$. The volume of the recirculation region represents approximately $90 \%$ or more of the total production of mixed fluid, and shows that the flux of mixed fluid from the turbulent zone is a decreasing function of time,

$$
Q=\frac{d V_{m}}{d t}=0.15 L_{C} h_{m}\left(L_{R} L_{f}\right)^{1 / 2} f^{1 / 2} t^{-1 / 2} .
$$

\subsection{Geostrophic transport calculations}

The observed distribution of dyed fluid (section 5.2.b) and the decrease in its rate of volume growth (5.11) describe a recirculation within the channel that cuts off the lateral entrainment of unmixed fluid and apparently curtails the production of mixed fluid. The dye distributions provide no direct information on vertical entrainment and are, moreover, difficult to quantify when instabilities and irregularities develop. The quantitative analysis thus covers only a relatively short time, generally less than 125 rotation periods, but the recirculation region continues to grow and the boundary current continues to carry mixed fluid into the basin interior. The vertical density profiles, described in section 5.3.b, cover the full duration of four experiments, and supplement the dye data with information about flow across a channel cross-section at all depths, not just the depth of the mixed layer.

Repeated conductivity probe measurements are made along a transect through the approximate center of the recirculation region. The location of the transect is sketched in Figure 5.15, and a typical set of vertical density profiles is shown in Figure 5.7. The profiles extend from approximately six $\mathrm{cm}$ below the rigid lid to within one-half $\mathrm{cm}$ of the sand bottom. Geostrophic velocities are calculated from the horizontal density differences between the profiles for four experiments with $N / f=\left[\frac{1}{4}, 1,3,9\right]$ and $L_{c} / L_{R}=[18,4.7,3,1]$. To do the calculations, it is assumed that geostrophy applies to flows away from the area of active mixing. The observed flows are slow and laminar, 
and the Rossby numbers of the swiftest flows in the boundary current are of the order, $O\left(10^{-2}\right.$ to $\left.10^{-1}\right)$. Geostrophy appears appropriate. It is further assumed that a level of no motion occurs at the base of the profiles. Finally, each cross channel transport calculation is adjusted to conserve volume. Density differences in the experiments are so small that conservation of mass does not provide an additional constraint on the geostrophic calculation. Therefore, net cross-channel volume transport is set to zero by assigning a uniform barotropic velocity to the entire flow field. In the absence of other independent information on the velocity field, a more realistic identification of barotropic velocity structure is not possible.

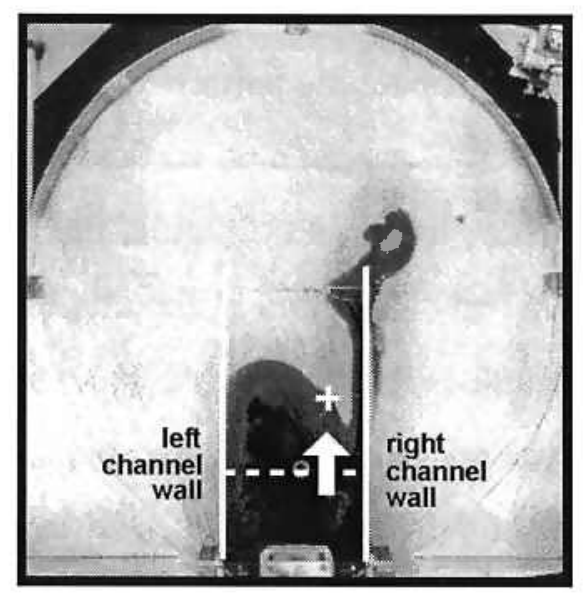

Figure 5.15: Diagram showing the location of the cross-channel probe transects, (dotted line). White arrow indicates the direction of positive flow, away from the mixing bar. Dye image from experiment with $(\omega, N, f)=(14,0.3,0.3) \mathrm{s}^{-1}, L_{C}=30.5 \mathrm{~cm}, N / f=1$, and $L_{C} / L_{R}=4.7$ at $t=900 \mathrm{~s}$.

The examples of along channel transport shown in Figures 5.16, 5.17 and 5.18 come from the experiment with $L_{C}=30.5 \mathrm{~cm},(\omega, N, f)=(14,0.33,0.31) \mathrm{s}^{-1}, N / f=1$ and $L_{C} / L_{R}=4.7$. These are the same as the parameters of the experiments pictured in Figures 5.7 and 5.15. Figure 5.16 shows four vertical profiles of horizontal along channel volume transport. The transport is integrated across the channel and adjusted for volume conservation. The profiles show a net flow outward at the level of the mixed layer (the 
positive direction in the figures), and a net flow toward the mixing zone above and below the mixed layer. Figure 5.17 shows details of the horizontal flow structure, integrated over a one $\mathrm{cm}$ depth, at the two levels indicated by shaded sections I and II in Figure 5.16. Section I is centered at the level of the mixing bar, $13.75 \mathrm{~cm}$ beneath the rigid lid. Section II is located below the mixed layer, at a depth of $17 \mathrm{~cm}$.

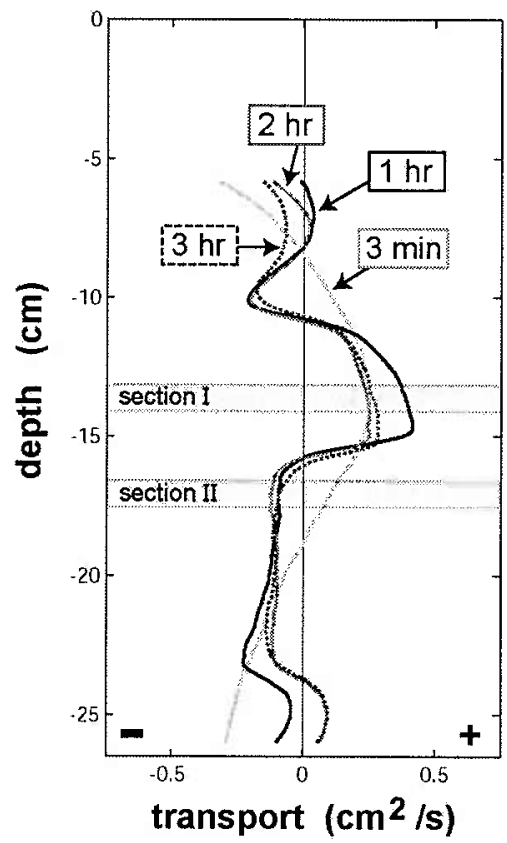

Figure 5.16: Horizontally integrated, along channel volume transport $\left(\mathrm{cm}^{2} / \mathrm{s}\right)$ vs depth $(\mathrm{cm})$ at location shown in Figure 5.15. Positive values are down channel, away from the mixing bar. Geostrophic calculation based on density data from experiment with $(\omega, N, f)=(14,0.3,0.3) \mathrm{s}^{-1}, L_{C}=30.5 \mathrm{~cm}, N / f=1$, and $L_{C} / L_{R}=4.7$. The transport profiles are for four times as indicated, $t=[3 \mathrm{~min}, 1 \mathrm{hr}, 2 \mathrm{hr}, 3 \mathrm{hr}$ ]. The shaded strips mark the location of the cross-channels details shown in Figure 5.17.

The four pairs of plots in Figure 5.17 display the volume transports through sections I and II for each of the four vertical profiles in Figure 5.16. The sense of the flow is the same at both levels, but there is a definite vertical shear. At $t=3 \mathrm{~min}$, the boundary current is just crossing the transect and the flow is positive at both levels, though weaker 
below the mixed layer. At later times, $t=1,2$, and 3 hrs, the net flow at middepth is positive and concentrated near the right wall, while the net flow at the lower level is inward and along the left wall. The flows imply that the export of fluid at the level of the mixed layer is being supplied in part by incoming unmixed fluid from the lower (and upper) levels. Hence, vertical entrainment is contributing to the flux of mixed fluid.
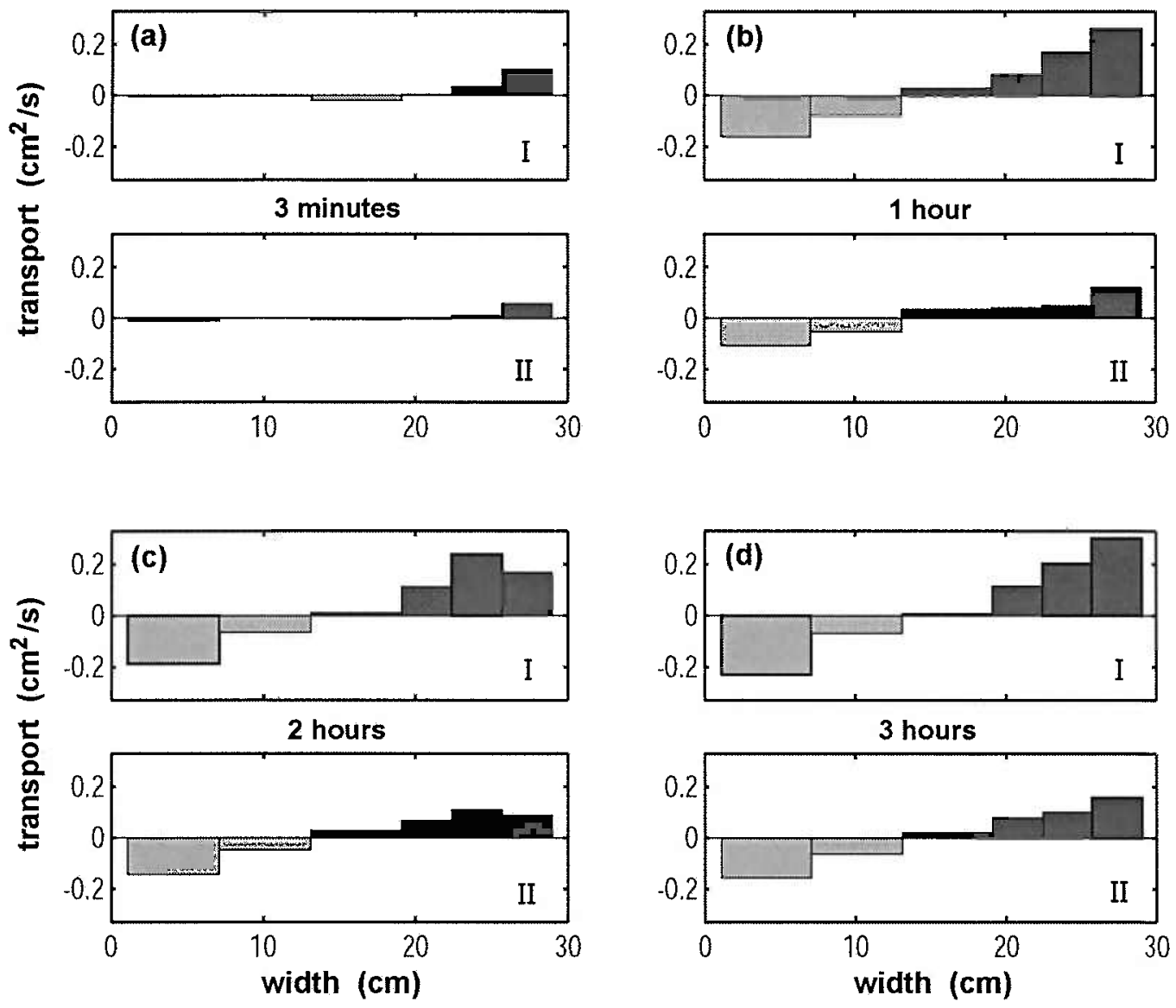

Figure 5.17: Vertically integrated, along channel volume transport $\left(\mathrm{cm}^{2} / \mathrm{s}\right)$ vs channel width $(\mathrm{cm})$, at location shown in Figure 5.15. Vertical integrations are over one $\mathrm{cm}$ depths centered $13.75 \mathrm{~cm}$ and $17 \mathrm{~cm}$ below the rigid lid, Section I and Section II, respectively. Positive values are down channel, away from the mixing bar. Geostrophic calculation based on density data from experiment with $(\omega, N, f)=(14,0.3,0.3) \mathrm{s}^{-1}$, $L_{C}=30.5 \mathrm{~cm}, N / f=1$, and $L_{c} / L_{R}=4.7$ (a) $\mathrm{t}=3 \mathrm{~min}$, (b) $\mathrm{t}=1 \mathrm{hr}$, (c) $\mathrm{t}=2 \mathrm{hr}$, (d) $\mathrm{t}=3 \mathrm{hr}$. 
Figure 5.18 plots the horizontally integrated along channel transport in Section I and II for each of the eleven transects made over the course of the three hour experiment. While the exact values of the geostrophic calculation rely on the earlier assumptions, the long term transport trend stands out. The dark gray diamonds show the adjusted geostrophic calculations for transport at middepth. They are superimposed on a curve representing the flux of mixed fluid from equation (5.12) for an equivalent one $\mathrm{cm}$ thick section of the mixed layer. The geostrophic calculations with the uniform barotropic correction are consistent with the predictions derived from the dye observations.

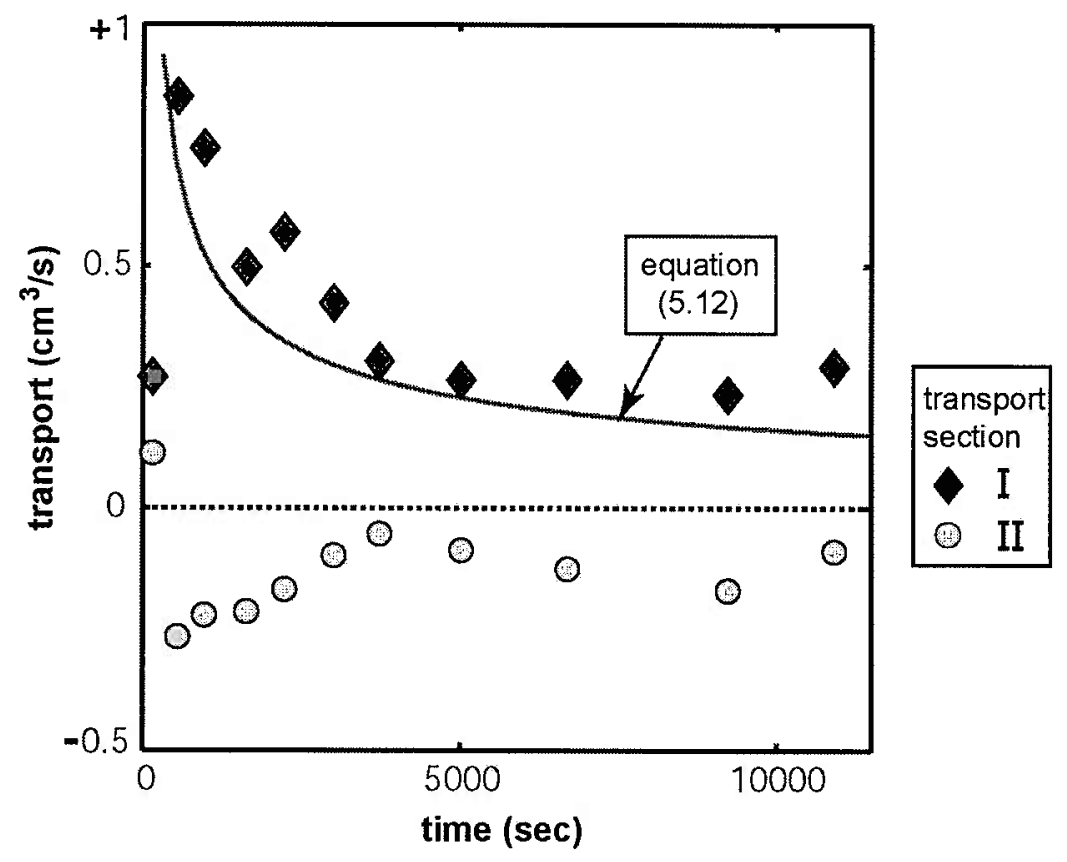

Figure 5.18: Along channel volume transport $\left(\mathrm{cm}^{3} / \mathrm{s}\right)$ vs time $(\mathrm{sec})$, at the location shown in Figure 5.15. The transport is horizontally integrated across the channel and vertically integrated over one $\mathrm{cm}$ depths centered $13.75 \mathrm{~cm}$ and $17 \mathrm{~cm}$ below the rigid lid, Section I and II, respectively. Positive values are down channel, away from the mixing bar. Geostrophic calculations based on density data from experiment with $(\omega, N, f)=(14,0.3,0.3) \mathrm{s}^{-1}, L_{C}=30.5 \mathrm{~cm}, N / f=1$, and $L_{C} / L_{R}=4.7$. Solid curve is mixed fluid flux from Equation (5.12). 
The data from the other three experiments are more scattered than that presented in Figure 5.18, but in all cases they show the recirculating flow strengthening over time and a net midlevel outflow declining with time. Figure 5.19 illustrates some qualitative differences between the two extremes of the parameter range. In panel $a$, the experiment with $N / f=9$ and $L_{C} / L_{R}=1$ shows a strong broad flow at middepth, out on the right and in on the left. In panel $\mathrm{b}$, the experiment with $N / f=\frac{1}{4}$ and $L_{C} / L_{R}=17$ shows a weaker flow and possibly two recirculating cells, a smaller more intense one near the right-hand boundary and a much slower wider one covering the left two-thirds of the channel.
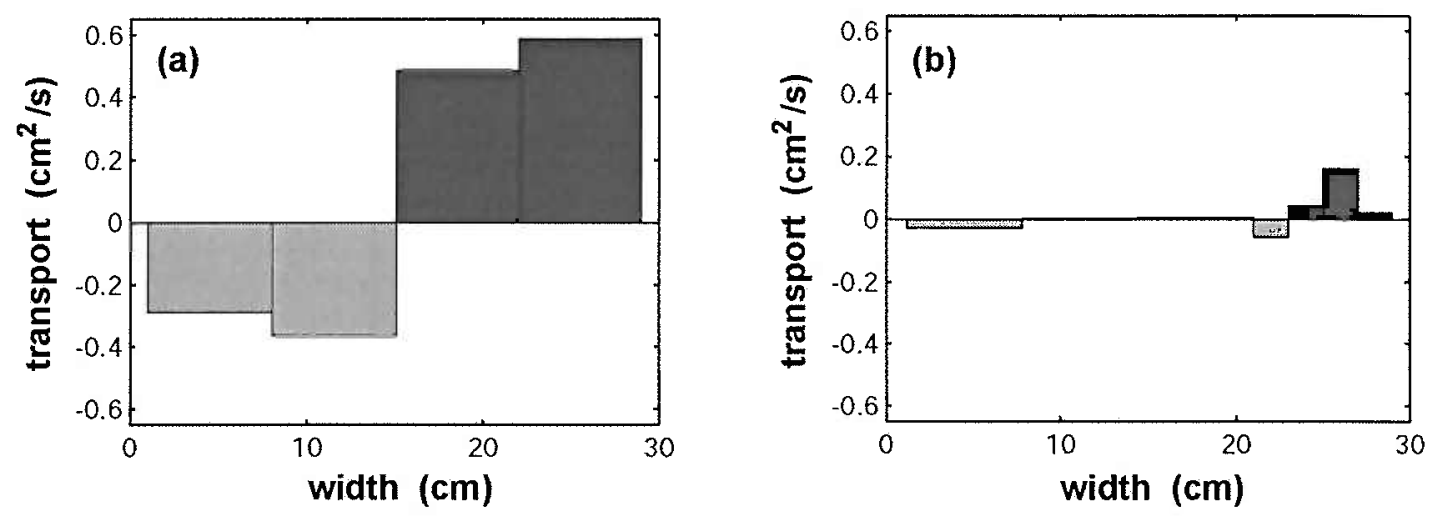

Figure 5.19: Vertically integrated, along channel volume transport $\left(\mathrm{cm}^{2} / \mathrm{s}\right)$ vs channel width $(\mathrm{cm})$, at location shown in Figure 5.15. Vertical integrations are over one $\mathrm{cm}$ depths centered $13.75 \mathrm{~cm}$ and $17 \mathrm{~cm}$ below the rigid lid, Section I and Section II, respectively. Positive values are down channel, away from the mixing bar. (a) Geostrophic calculation based on density data from experiment with $(\omega, N, f)=(14,0.9$, $0.1) \mathrm{s}^{-1}, L_{C}=30.5 \mathrm{~cm}, N / f=9$, and $L_{c} / L_{R}=1 . \mathrm{t}=80 \mathrm{~min}$. (b) Geostrophic calculation based on density data from experiment with $(\omega, N, f)=(14,0.3,1.2) \mathrm{s}^{-1}, L_{C}=30.5 \mathrm{~cm}, N / f=\frac{1}{4}$, and $L_{C} / L_{R}=18, \mathrm{t}=50 \mathrm{~min}$.

\subsection{Mixing in the absence of rotation: $f=0$}

The rotating, stratified experiments discussed so far investigate laterally confined mixing in fluids with $\frac{1}{4} \leq N / f \leq 9$. While local vertical mixing and the height of the mixed layer 
near the turbulent zone are found to be independent of rotation, the volume production of mixed fluid is a function of both rotation and stratification. The empirical formula for the volume flux of mixed fluid, (5.12), can be rewritten in terms of the external parameters, $\omega, N$ and $f$, using the formula for $h_{m}$ (5.4), and the definitions of the length scales, $L_{R}(5.1)$ and $L_{f}(5.3)$,

$$
Q \sim L_{C} \omega(N f)^{-1 / 4} t^{-1 / 2}
$$

For a given channel width $L_{C}$, stratification $N$, and mixing intensity, represented here by the bar frequency $\omega$, the effect of increasing the rotation rate $f$ will be to decrease the production of mixed fluid. Conversely, production increases as the rotation rate is reduced. The patterns of mixed fluid export also undergo a transition for $f \leq 0.2 \mathrm{~s}^{-1}$, $3<N / f \leq 9$ in these experiments. The narrow boundary current broadens and carries a significantly greater percentage of the mixed fluid out of the channel into the basin interior.

This section looks at the limit $f=0$, in order to investigate the dynamics of mixed fluid production as $f \rightarrow 0$. The results from four stratified nonrotating experiments are presented. Three experiments are conducted using the original channel width, $L_{C}=30.5 \mathrm{~cm}$, with $N=[0.33,0.62,0.89] \mathrm{s}^{-1}$, and one, with $N=0.88 \mathrm{~s}^{-1}$, uses the 20.3 cm channel.

The temporal mean mixed layer heights are designated by circular symbols in the plots in Figures 5.6 and 5.11. In three out of the four nonrotating experiments, the heights are on the low side, approximately $90 \%$ of the values predicted by the overall empirical relation for $h_{m},(5.4)$. The heights are measured four $\mathrm{cm}$ in front of the mixing bar, but in the absence of rotation, turbulent fluid collapses into a thinning intrusion. Higher values would have been recorded closer to the turbulent zone.

The volumes are estimated using spatial mean heights $\bar{h}$, calculated from the intrusion side view area and length. It is assumed that the cross channel layer thickness is uniform in the nonrotating intrusion. The volume data are shown in Figure 5.20. Panel $a$ 
plots the dimensional data, and panel $b$ shows the data scaled by $L_{C} h_{m}{ }^{2}$ versus the nondimensional time $N t$. For unconfined vertical boundary mixing, data from the nonrotating experiments collapse when normalized by $L_{B} h_{m}{ }^{2}$, where $L_{B}$ is the bar length. Data from the laterally confined channel experiments do not collapse when normalized by $L_{C} h_{m}{ }^{2}$. Instead, the volume scales with $h_{m}{ }^{3} N t$. Figure 5.21 plots the volume, normalized by $h_{m}{ }^{3}$, versus $N t$. A straight line fit to the data gives

$$
V_{m}=(0.21 \pm 0.06) h_{m}^{3} N t
$$

The volume of mixed fluid is unexpectedly independent of the channel width.
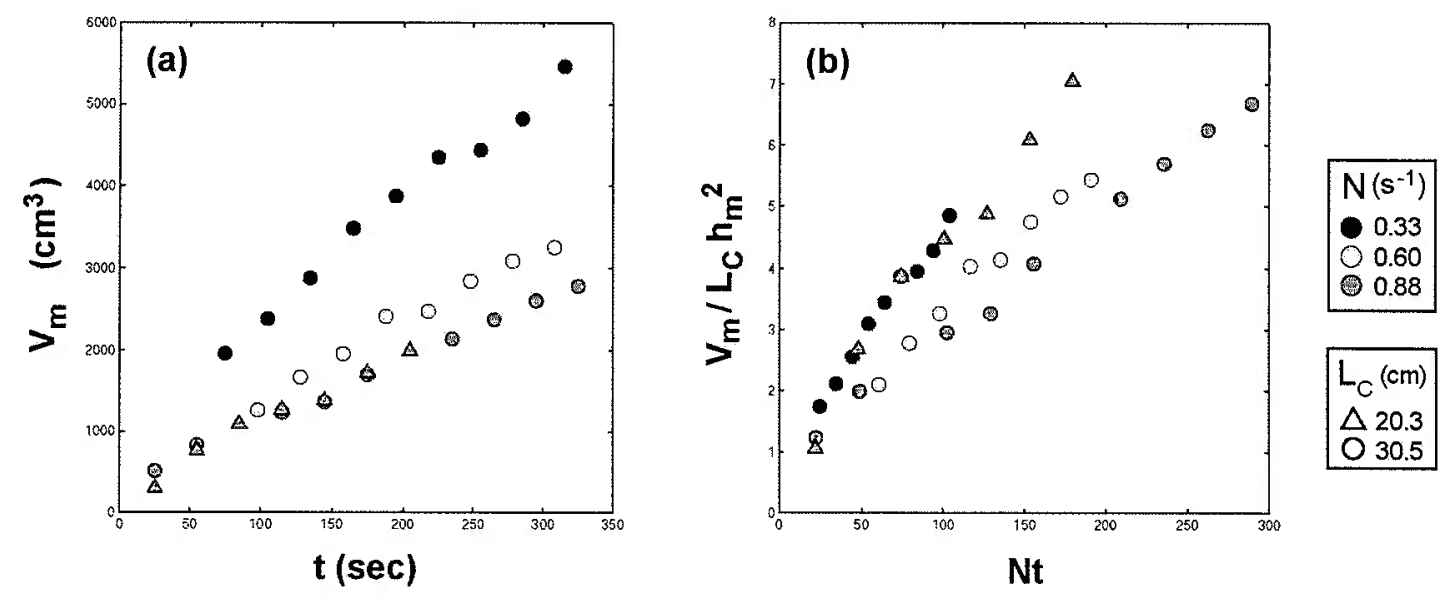

Figure 5.20: Volume of mixed fluid in experiments without rotation. Symbols indicate channel width and stratification. (a) Volume $\left(\mathrm{cm}^{3}\right)$ vs time (sec), (b) volume normalized by $L_{C} h_{m}{ }^{2}$ vs nondimensional time $N$. 


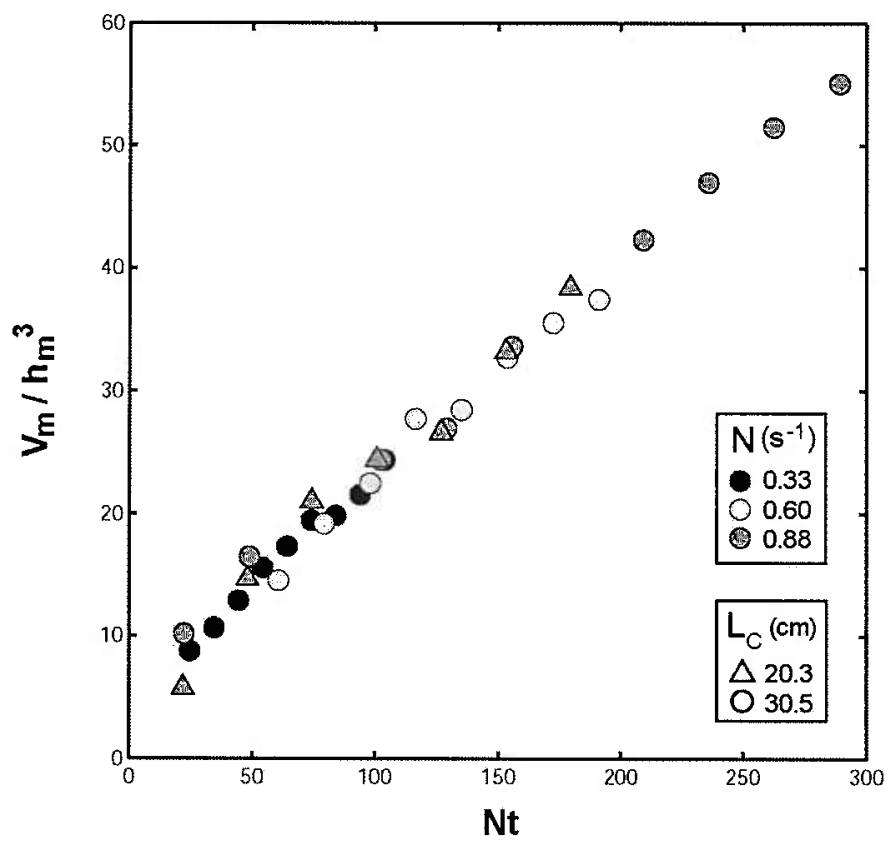

Figure 5.21: Volume of mixed fluid in experiments without rotation. Symbols indicate channel width and stratification. Volume normalized by $h_{m}{ }^{3}$ vs nondimensional time $N t$.

The scaling of the flux in the unconfined experiments, $Q \sim L_{B} N h_{m}{ }^{2}$ (4.22), is consistent with a mixed fluid production that is driven by the collapse of the turbulent layer into the interior. The entrainment of unmixed fluid, from whatever source, lateral as well as vertical, is sufficient to supply a steady export of mixed fluid across the face of the turbulent zone with area $A=L_{B} \times h_{m}$ at a speed proportional to a gravity current, $N h_{m}$. The supply of unmixed fluid is reduced in the confined experiments, where channel walls block lateral entrainment at the sides of the turbulent zone. Nevertheless, the channel width was expected to appear in the scaling of mixed fluid production through its role in setting the dimensions for vertical entrainment across the top and bottom surfaces of the turbulent zone, and for possible horizontal entrainment along the upper and lower edges of the collapsing front as described by DeSilva and Fernando (1998). 
In the nonrotating, closed container experiments discussed in Chapter 2.3 (Ivey and Corcos, 1982; Thorpe, 1982; Browand and Hopfinger, 1985; Maxworthy and Monismith, 1988; DeSilva and Fernando, 1998), the production of mixed fluid is treated as a two dimensional process of entrainment and detrainment per unit width. The model of vertical entrainment developed in Chapter 4 takes a two-dimensional approach and calculates mixed fluid flux from the entrainment of unmixed fluid, with a velocity $w_{e}$, across the upper and lower surfaces of the turbulent zone. Here the surface areas are given by $A=L_{C} \times h_{m}$, with length set by the channel and width proportional to the height of the mixed layer, $h_{m}$. Maximum values for vertical entrainment are obtained when the velocity $w_{e}$ is evaulated at the time that the mixed layer height is established, at $t \approx 100 \mathrm{~s}$. The method is explained in Chapter 4.4.b. Calculations for the experiments with $L_{C}=20.3 \mathrm{~cm}, N=0.88 \mathrm{~s}^{-1}$ and with $L_{C}=30.5 \mathrm{~cm}, N=0.33 \mathrm{~s}^{-1}$ produce volumes that range from $8 \%$ to $16 \%$ of the actual measurements. The greater magnitude of observed mixed fluid production and the absence of channel width, $L_{C}$, from the scaling (5.14), indicate that lateral entrainment remains important in the present experiments. In this configuration, where mixed fluid is able to exit the channel and enter a larger basin, mixing appears to be a three dimensional process.

In the presence of rotation, channel width becomes a factor in the volume flux of mixed fluid (5.12). However, the most significant difference between the rotating and nonrotating experiments is the time dependence of the flux. In the nonrotating experiments, the flux $\mathrm{Q}$ is constant,

$$
\frac{d V_{m}}{d t}=Q=(0.21 \pm 0.06) N h_{m}{ }^{3} .
$$

For $f=0$, the production of mixed fluid is nearly linear. For $f>0$, the growing recirculation region blocks lateral access to the turbulent zone and the rate of volume growth declines with time. Figure 5.22 compares the dimensional volume growth for four experiments with and without rotation. The experiments have the same channel width, bar frequency and stratification, $L_{C}=30.5 \mathrm{~cm}$, and $(\omega, N)=(14,0.6) \mathrm{s}^{-1}$, but 
different rotation rates, $f=[0,0.3,0.6,1.2] \mathrm{s}^{-1}$. The experimental observations are superimposed on the growth curves predicted by the empirical data fits given by equations (5.11) and (5.14). The results clearly demonstrate the restrictive effect of the $t^{1 / 2}$ dependence on the volume growth of the rotating experiments compared to the nonrotating experiment.

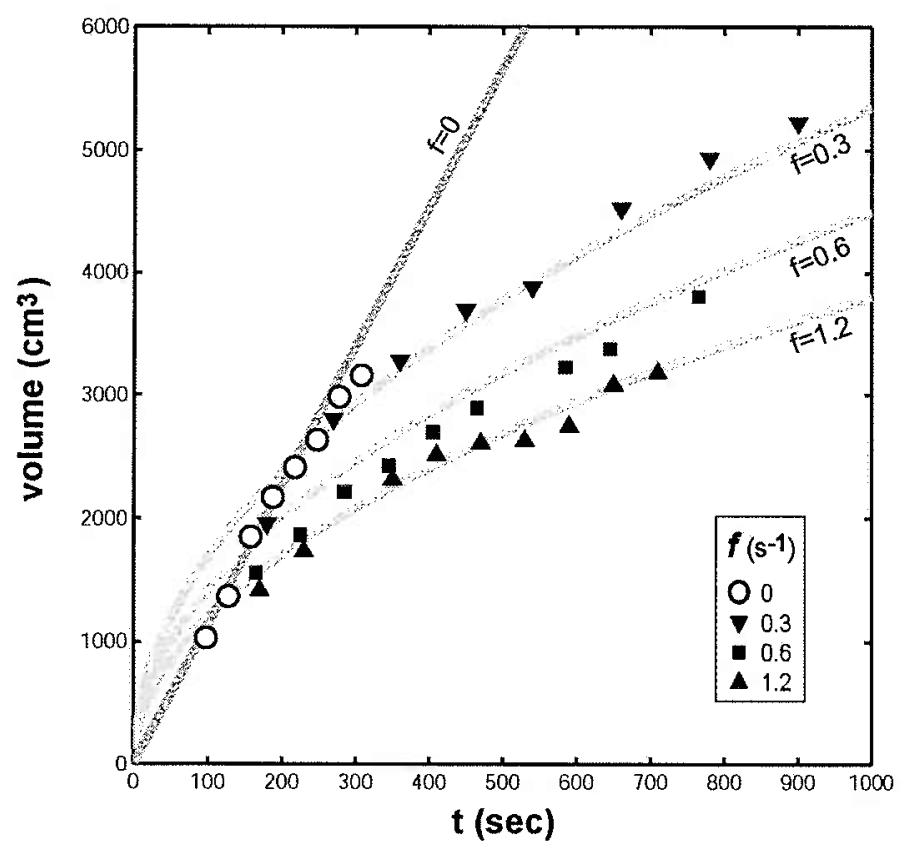

Figure 5.22: Volume of mixed fluid in experiments with and without rotation. Volume $\left(\mathrm{cm}^{3}\right)$ vs time (sec). All experiments have $(\omega, N)=(14,0.6) \mathrm{s}^{-1}$ and $L_{C}=30.5 \mathrm{~cm}$. The solid lines are volume growth predicted by the empirically derived equations (5.11) and (5.14). The symbols are data points from the experiments with $f=[0,0.3,0.6,1.2] \mathrm{s}^{-1}$.

\subsection{Summary}

The laboratory study described in this chapter investigates the interaction between a body of rotating stratified fluid and a patch of turbulent mixing confined on three sides at the end of a channel. The laboratory measurements of mixed layer height, lateral area and 
total volume are evaluated in terms of the channel width, $L_{C}$, and three basic length scales, $H_{N}(5.2), L_{f}(5.3)$ and $L_{R}(5.1)$, each of which describes an aspect of the underlying physics of mixing. The first two, the buoyancy and rotational length scales, are related to the interaction of turbulence with the ambient fluid, and the third, the deformation radius, is related to the adjustment of the mixed fluid outflow to background rotation. The experiments are characterized by the nondimensional parameters, $N / f$ and $L_{C} / L_{R}$, and cover the range, $\frac{1}{4} \leq N / f \leq 9$ and $1 \leq\left(L_{C} / L_{R}\right) \leq 18$. The experimental results illustrate the contribution of $N$ and $f$ to the production and distribution of mixed fluid, and emphasize the interactive effects between the channel walls and rotationally influenced horizontal circulations.

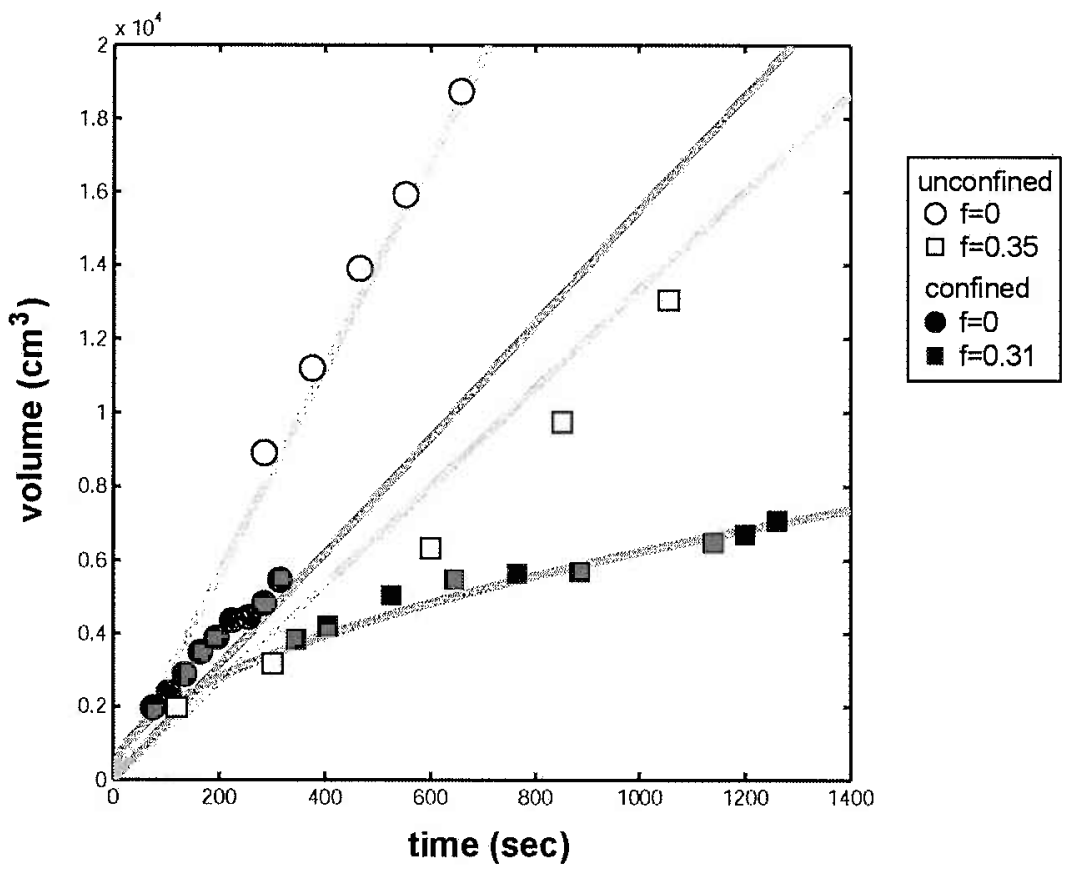

Figure 5.23: Volume of mixed fluid in unconfined and laterally confined experiments with $f=[0,0.3] \mathrm{s}^{-1}$. Volume $\left(\mathrm{cm}^{3}\right)$ vs time (sec). All experiments have $(\omega, N)=(14,0.3) \mathrm{s}^{-1}$ and $\left(L_{B}, L_{C}\right)=30.5 \mathrm{~cm}$. The solid lines are volume growth predicted by the empirically derived equations, (4.21), (4.9), (5.11) and (5.14). The symbols are data points from individual experiments. 
The mixed layer height, which is quickly established and remains nearly steady over time, scales with $H_{N}$ and is a function of the strength of the mixing and the ambient stratification. Neither rotation nor the presence of the channel walls affects the vertical scale of the mixing. The overall production of mixed fluid, however, is strongly affected by the lateral confinement of the mixing region. Figure 5.23 compares the volume production due to unconfined vertical boundary mixing and confined channel mixing. Measured and predicted volumes are shown for four experiments, two each for unconfined and confined mixing, with $f=0$ and $f=0.3 \mathrm{~s}^{-1}$. The bar and buoyancy frequencies are the same in all four, $(\omega, N)=(14,0.3) \mathrm{s}^{-1}$, as is the length of the mixing region, $\left(L_{C}, L_{B}\right)=30.5 \mathrm{~cm}$. The presence of the channel walls reduces production of mixed fluid in both the nonrotating and rotating experiments.

The $t^{-1 / 2}$ time dependence of mixed fluid flux (5.12) is what distinguishes the laterally confined rotating experiments from the nonrotating and unconfined rotating experiments. Not only do the channel walls eliminate direct access to the sides of the mixing region in the rotating case, but they also promote the formation of a boundary current along the right-hand wall and a growing recirculation region in front of the mixing bar. As the recirculating cell spreads across the channel it restricts the inflow of stratified fluid to the mixing region. The dye distribution suggests that the channel is eventually blocked by the recirculating mixed fluid, even in the experiments with the highest values of $L_{C} / L_{R}$. However, the dyed region in front of the bar continues to expand outward until it nearly fills the length of the channel, and dyed fluid continues to exit the channel via the boundary current (see, for example, Figure $5.3 f$ ). Both phenomena indicate the continued production of mixed fluid. While some stratified fluid may still flow in towards the turbulent zone at the level of the mixed layer, the geostrophic calculations of section 5.4 indicate that turbulent mixing relies in part on vertical entrainment. They show a persistent but decreasing net outflux at the level of the mixed layer and a net influx below (and above) it. The calculations also indicate a persistent horizontal circulation within the channel (see Figure 5.17). This result is consistent with dye images 
which show a defined region of mixed fluid remaining within the channel over the duration of the experiments. With the recirculating cells confined to the channel, the boundary currrent forms the sole conduit for distributing mixed fluid into the interior of the basin.

An important lesson from the experiments is that rough topographic features that promote turbulence can also limit the entrainment of unmixed fluid into the turbulent zone and thus restrict the production of mixed fluid. In the experiments where $f=0$, mixed fluid production is reduced, but the mixed layer flows directly out of the channel into the larger basin. In the experiments where $f>0$, the development of a recirculating flow traps mixed fluid within the channel. Not only is mixed fluid production reduced, but the rate of production decreases in time and the advection of mixed fluid into basin is limited to the boundary current. The extent to which this happens is a function of $\mathrm{N} / \mathrm{f}$ and $L_{C} / L_{R}$.

In the experiments with $\frac{1}{4} \leq N / f \leq 3$ and $3 \leq L_{C} / L_{R} \leq 18$ the boundary current carries only $1 \%$ to $4 \%$ of the short term mixed fluid production. As $N / f$ increases and $L_{C} / L_{R}$ decreases, the boundary current becomes more important. In the experiments with $4.5 \leq N / f \leq 9$ and $1 \leq L_{C} / L_{R} \leq 2$ the amount of mixed fluid carried by the boundary current jumps to more than $10 \%$, and a broader flow of mixed fluid exits the channel and contributes to the density distribution in the larger basin. 


\section{Chapter 6}

\section{Discussion}

This research has been motivated by the observed uneven distribution of mixing in the ocean and by the idea that an overall advective-diffusive balance could be achieved through the horizontal advection of locally mixed fluid (Equation 1.2). The thesis addresses the question of fluid exchange between a region of intense localized mixing and an ambient body of rotating stratified water, and investigates the transport of mixed, but no longer turbulent, water from the boundary of a basin into the interior.

\subsection{Localized mixing in the laboratory}

The phenomena are investigated in a laboratory process study. The experimental approach was taken in order to control and parameterize the turbulence, and isolate the effects of rotation and stratification on the evolution of mixed fluid. The work was done in the context of earlier laboratory studies of turbulent mixing, reviewed in Chapter 2. The discussion that follows identifies the unique aspects of the present research and summarizes its contributions.

As in the earlier laboratory experiments, mixing is imposed by mechanical means. The main difference here is that the mixing is highly localized. The mechanism is a single vertically oscillating bar of finite horizontal length, located at middepth at the boundary of a large body of stratified salt water. It generates a limited turbulent zone. In the vertical boundary mixing experiments of Chapter 4 the turbulent zone is exposed on 
three sides and open to both vertical and horizontal exchanges with the ambient fluid. In the channel experiments of Chapter 5 the sides of the mixing bar are laterally confined between the channel walls, but the channel itself opens into a larger basin. Both sets of experiments are designed to avoid having the closed nature of the laboratory tank affect the mixing process or the export of mixed fluid.

The single bar mixing mechanism is distinct from the meshed and multibar grids of earlier experimenters, but it is shown to be effective in producing turbulent fluid motion. The exploratory experiments discussed in Chapter 3.3 establish a parameterization of the bar-induced turbulent velocity, $u_{t}$ (3.5), and rms length scale, $l_{t}(3.6)$. The properties are not measured directly, but are inferred from the development of the turbulent zone and the evolution of the mixed layer following the methodology of Thorpe (1982). Both $u_{t}$ and $l_{t}$ are functions of distance from the mixer. The bar parameterization is used to derive length scales which relate the spatial decay of turbulent motion to the onset of buoyant and rotational effects. The buoyancy scale height, $H_{N}(3.13)$, is the distance from the mixing bar at which stratification is expected to inhibit vertical turbulent overturning. The rotational length scale, $L_{f}(3.14)$, is the distance from the mixing bar at which rotation is expected to inhibit the horizontal turbulent motions.

The interaction of bar-induced turbulence and stratification produces a single mixed layer. The layer heights, $h_{m}$, measured adjacent to the turbulent zone, are approximately equal to $H_{N}$ in both the vertical boundary mixing and channel experiments. The height is solely a function of the buoyant suppression of turbulence, and independent of rotation. This result is consistent with the experimental findings of earlier studies with and without rotation. In those studies, however, the export of mixed fluid from the turbulent zone was soon blocked by the tank and mixed layer growth resumed. In the present experiments the mixed layer maintains a steady height. In the presence of rotation, moreover, it is spatially uniform.

The extent of vertical mixing is a robust function of the buoyancy scale height. It is unaffected by the lateral confinement of the turbulent zone in the channel. It is 
independent of rotation over the parameter range, $\frac{1}{4} \leq N / f \leq 9$, and independent of bar length, $L_{B}$, where $\frac{1}{8} \leq h_{m} / L_{B} \leq \frac{1}{3}$.

The present research emphasizes the combined effects of rotation and stratification on localized mixing, and the experimental results suggest that the rotational inhibition of turbulence is more important than previously recognized. Where buoyancy affects vertical overturning motions, rotation acts on motions normal to the axis of rotation. In the experiments the turbulent zone width is not measurable in the same way as the height. It is obscured by the gravitational collapse and export of mixed fluid. However, the rotational length scale, $L_{f}$, does appear consistently in scaling the horizontal distribution of mixed fluid, as well as in the lateral spread of disturbed fluid in the unstratified experiments. In Chapter 4.4 the volume flux of mixed fluid in the vertical boundary mixing experiments is described by a model of lateral entrainment into the sides of a turbulent zone with dimensions set vertically by $H_{N}$ and horizontally by $L_{f}$. In the laterally confined channel experiments the area and volume scale as $L_{f}{ }^{1 / 2}$ and grow as $t^{1 / 2}$. This scaling is attributed to the curtailment of lateral entrainment as the channel becomes blocked with recirculating mixed fluid.

The emergence of $L_{f}$ as an important experimental scale is tied to the entrainment of unmixed fluid into the turbulent zone. It is not surprising that fluid would preferentially follow isopycnals, but the previous one and two-dimensional laboratory studies precluded lateral entrainment. The present study includes four categories of experiments, nonrotating and rotating, unconfined and laterally confined. They are illustrated by the examples in Figure 5.23. The greatest outflux of mixed fluid occurs in the unconfined nonrotating experiments. The production rate may be determined by the horizontal pressure gradient at the face of the turbulent zone and unlimited by restrictions on the entrainment of unmixed fluid. Both confinement by the channel walls and the presence of rotation limit lateral entrainment and reduce the volume of mixed fluid. As long as lateral entrainment remains possible, vertical entrainment appears to make a minor 
contribution. However, in the rotating channel experiments vertical entrainment becomes the determining factor over the long term as the lateral entrainment of unmixed fluid is progressively reduced.

The effects of entrainment on the production of mixed fluid demonstrate how active mixing is dynamically different from the passive release of mixed fluid into the environment. The entrainment patterns also influence the advection of mixed fluid into the interior of the basin. In the presence of rotation the export of mixed fluid from the patch of vertical boundary mixing was expected to turn to the right and form a boundary current. Instead, entraining flows are observed on both sides of the mixing region and the mixed fluid moves out directly into the tank as a radial front until its cross-tank progress is arrested. Further export of mixed fluid into the interior is through eddies. In the laterally confined experiments the immediate presence of the channel wall promotes the formation of a boundary current. Initially it carries only a minor percentage of the exported fluid. The majority of the outflux develops into a recirculating cell which gradually fills the channel, restricts horizontal inflow of unmixed fluid and reduces the production of mixed fluid. The recirculating fluid remains within the channel and the small boundary current is the sole conduit for mixed fluid exiting the channel and entering the larger basin.

The present experiments are unique in the localization of imposed mixing and in the quantification of mixed fluid volume. As a result they emphasize the role of lateral entrainment and bring out the importance of rotation in the production and distribution of mixed fluid. In addition, a comparison of unconfined and laterally confined mixing demonstrates the role of topography in mixing productivity and in the advection of mixed fluid into the larger basin.

\subsection{Localized mixing in the ocean}

The main external parameters in the laboratory experiments are the stratification $N$, the rotation rate $f$, the strength of the imposed mixing, represented by the bar frequency $\omega$, 
and the two geometric configurations. In the discussion that follows the laboratory variables are placed in an oceanographic context in order to assess the applicability of experimental results to ocean observations.

The mixing mechanism employed in the laboratory provides a reliable source of controlled sustained turbulence, and the properties of bar-generated turbulence are parameterized and used to evaluate the experimental results. If these results are to be applied to ocean observations, the mixing terms need to be reformulated as quantities measurable in the ocean.

One approach is described in the conclusion to Chapter 4 . Since the mixed layer height $h_{m}$, given by (4.2) and (5.4), is a robust function of the buoyancy height scale $H_{N}$ (3.13), the magnitude of the height can be serve as a surrogate for mixing intensity. The rotational length scale (3.14), as a function of layer height (4.2), is

$$
L_{f}=\left(\frac{N}{f}\right)^{1 / 2} h_{m}
$$

By definition (3.15), the deformation radius, based on the mixed layer height, is

$$
L_{R}=\frac{N}{f} h_{m}
$$

For oceanographic applications, $N$ and $f$ are the local buoyancy and Coriolis frequencies, respectively. The mixed layer thickness $h_{m}$ can be estimated from the Thorpe scale of turbulent overturning, $L_{T}$. The scale (Thorpe 1977) is empirically derived from an ordering and averaging of displacements in the vertical density profile. With the equations for $L_{f}$ (6.1) and $L_{R}(6.2)$, observations of $N, f$ and $h_{m}$ provide the necessary information for predicting flux and areal spreading. The flux for unconfined mixing (4.10) is

$$
Q_{\text {unconfined }}=0.16 h_{m}{ }^{3}\left(\frac{N}{f}\right)^{3 / 2} f .
$$

For confined mixing, the flux (5.12), 


$$
Q_{\text {confined }}=0.14 h_{m}{ }^{2}\left(\frac{N}{f}\right)^{3 / 4} L_{C} f^{1 / 2} t^{-1 / 2},
$$

where $L_{C}$ is the width of the confining topography. Similar substitutions can be made in the formulae for the areal spreading of mixed fluid, (4.6) and (5.6). The coffficients in (6.3) and (6.4) and those used in the rest of this chapter are based on the constants of proportionality empirically determined in the laboratory and they depend in part on the specifics of the mixing mechanism. It is recognized that they are being applied to a different mixing mechanism when they are used to interpret oceanographic data.

A second approach estimates mixed layer heights from dissipation rate measurements. The dissipation rate of turbulent kinetic energy, $\varepsilon=u_{t}^{3} / l_{t}(2.9)$, is related to layer height through the Ozmidov scale, $L_{O}=\left(\varepsilon / N^{3}\right)^{1 / 2}$ (2.8). In the present laboratory study, dissipation rates are not measured directly, but the turbulent velocity and length scales, $u_{t}$ and $l_{t}$, are derived from a model based on the Ozmidov scaling (see Chapter 3.3 and 3.5). Consequently, the buoyancy scale height $H_{N}$ and thus the mixed layer height $h_{m}$ can be described as functions of $L_{O}$. The laboratory results give $h_{m} / L_{O} \approx 4$,

$$
h_{m} \approx 4\left(\frac{\varepsilon}{N^{3}}\right)^{1 / 2}
$$

Previous laboratory studies (Thorpe, 1982; Browand and Hopfinger, 1985; Davies et al., 1991; De Silva and Fernando, 1998) have found values between 1 and 5 for the ratio of critical height to Ozmidov length. A similar relationship was also established in a study of wind-driven mixing in a fresh water lake. Dillon (1982) used density profiles and microstructure measurements to compare the Thorpe scale, $L_{T}$, and Ozmidov scale, $L_{O}$. Turbulent overturns were small near the source of mixing, $L_{T} \ll L_{O}$, but away from the surface, they showed an $O(1)$ correspondence to the Ozmidov scale with $L_{T}=1.25 L_{O}$.

Oceanic microstructure observations are reported either as dissipation rates or as inferred diapycnal diffusivities, with the diffusivity, 


$$
K_{\rho}=\Gamma\left(\frac{\varepsilon}{N^{2}}\right)
$$

where $\Gamma$ is the mixing efficiency, commonly evaluated as 0.20 or 0.25 (St. Laurent et al., 2001; Toole et al., 1994). When the mixed layer height is written in terms of diffusivity,

$$
h_{m} \approx 4 \Gamma^{-1 / 2}\left(\frac{K_{\rho}}{N}\right)^{1 / 2}
$$

$K_{\rho}$ is the functional equivalent of the grid action (2.5) and $h_{m}$ appears in a form that is analogous to the equilibrium height in grid-mixing experiments (2.12).

In the discussion that follows $N, f$ and $\varepsilon$ data are taken from three studies of ocean mixing and used to evaluate the flux of mixed fluid and the extent of its vertical and horizontal distribution. Mixed layer height is calculated from equation (6.5), and the rotational length scale and the deformation radius are from equations (6.1) and (6.2). The flux of mixed fluid for unconfined vertical boundary mixing is given by

$$
Q_{\text {unconfined }} \approx 10\left(\frac{\varepsilon}{N^{3}}\right)^{3 / 2}\left(\frac{N}{f}\right)^{3 / 2} f
$$

and the flux for laterally confined channel mixing is

$$
Q_{\text {confined }} \approx 2\left(\frac{\varepsilon}{N^{3}}\right)\left(\frac{N}{f}\right)^{3 / 4} L_{C} f^{1 / 2} t^{-1 / 2}
$$

Table 6.1 shows representative values of buoyancy frequencies and dissipation rates taken from profiles of $N^{2}$ and $\varepsilon$ as functions of depth (or distance above bottom) for four topographic categories: the canyon and crest of a transverse fracture zone of the MidAtlantic Ridge located in the Brazil Basin at $22^{\circ} \mathrm{S}$ (Polzin et al., 1997; Ledwell et al., 2000 ; St. Laurent et al., 2001), the flank of the Fieberling Seamount at $32^{\circ} 26^{\prime} \mathrm{N}$ in the North Pacific (Kunze and Toole, 1997; Toole et al., 1997) and a canyon on the continental slope off North Carolina at $36^{\circ} 30^{\prime} \mathrm{N}$ (Polzin and Toole, 2002). The table gives the calculations for $N / f, h_{m}(6.5), L_{f}(6.1)$ and $L_{R}$ (6.2).

Much of the diapycnal diffusivity, inferred from observations in these regions, is attributed to internal waves which reflect upward off rough topography, and interact and 
break at some distance from the boundary. The present research has focussed specifically on vertical boundary mixing and the experimental results do not necessarily describe the dynamics of turbulent patches generated in the interior. The critical characteristics of the boundary experiments are the horizontal interface between the defined turbulent zone and the surrounding fluid, and the horizontal circulation set up by the collapse of mixed fluid into the interior and the lateral entrainment of unmixed fluid into the mixing region. This qualitative picture should apply to sloping as well as vertical boundaries. The data in Table 6.1 are treated as descriptions of conditions at the boundary.

\begin{tabular}{|l|r|r|r|r|r|r|r|}
\hline Site (depth $[\mathrm{m}])$ & $\mathrm{N}\left(\mathrm{s}^{-1}\right)$ & $\mathrm{f}\left(\mathrm{s}^{-1}\right)$ & $\varepsilon\left(\mathrm{m}^{2} / \mathrm{s}^{3}\right)$ & $\mathrm{N} / \mathrm{f}$ & $\mathrm{h}_{\mathrm{m}}(\mathrm{m})$ & $\mathrm{L}_{\mathrm{R}}(\mathrm{m})$ & $\mathrm{L}_{\mathrm{f}}(\mathrm{m})$ \\
\hline $\begin{array}{l}\text { MAR } \\
\text { crest }\end{array}(4300)$ & $1 \times 10^{-3}$ & $5.4 \times 10^{-5}$ & $1.4 \times 10^{-9}$ & 19 & 5 & 90 & 20 \\
\hline $\begin{array}{l}\text { MAR } \\
\text { canyon }(4550)\end{array}$ & $7.7 \times 10^{-4}$ & $5.4 \times 10^{-5}$ & $2 \times 10^{-9}$ & 14 & 9 & 120 & 30 \\
\hline $\begin{array}{l}\text { Seamount } \\
\text { flank (1300) }\end{array}$ & $2.2 \times 10^{-3}$ & $7.8 \times 10^{-5}$ & $4.5 \times 10^{-9}$ & 29 & 2.5 & 70 & 15 \\
\hline $\begin{array}{l}\text { Continental slope } \\
\text { canyon (1100) }\end{array}$ & $1 \times 10^{-3}$ & $8.7 \times 10^{-5}$ & $6 \times 10^{-9}$ & 12 & 10 & 110 & 35 \\
\hline
\end{tabular}

Table 6.1: Calculation of mixed layer height, $h_{m}$, deformation radius, $L_{R}$, and rotational length scale, $L_{f}$, from observed buoyancy frequency, $N$, Coriolis frequency, $f$, and kinetic energy dissipation rate, $\varepsilon$.

$N / f$ in the selected regions is an order of magnitude greater than that explored in the unconfined laboratory experiments which cover $\frac{1}{4} \leq N / f \leq 2$. However, a comparison between experiments with and without rotation (Chapter 4.5) indicates that the relationship between rotation and mixed fluid production is a function not of $N / f$ directly, but of $L_{f} / L_{B}$, the ratio of the rotational length scale (3.14) to the length of the mixing region given by the bar length $L_{B}$. In the channel experiments $N / f$ is extended to $O(10)$, and reaches the values found in both the MAR and continental slope canyons. 
The mixed layer height $h_{m}$ is in all cases less than $10 \mathrm{~m}$ thick. The layer height associated with the seamount flank, $2.5 \mathrm{~m}$, is comparable to the overturning scales observed by Toole et al. (1997), which were of order $1 \mathrm{~m}$ with a maximum of $4 \mathrm{~m}$. At each of the four ocean sites in Table 6.1 the vertical distribution of dissipation is much greater than the individual layer height. Significant dissipation rates are found over the heights listed in Table 6.2 under $\mathrm{H}_{\text {region. }}$. Experimental studies have shown that turbulent mixing over extended vertical distances produces multiple layers of mixed fluid. The grid mixing experiments of Ivey and Corcos (1982), Thorpe (1982), and Browand and Hopfinger (1985) generated multiple layers, each of which was proportional to the Ozmidov scale. Multiple horizontal intrusions were also observed in the rod stirring experiments of Park et al. (1994), and Perera et al. (1995) and in laboratory studies of wave breaking on sloping boundaries (Cacchione and Wunsch, 1974; Ivey and Nokes, 1989). While the calculations in Table 6.1 are for individual layers at specific depths, they may be considered representative of series of vertically stacked layers.

With $h_{m}<10 \mathrm{~m}$ and $N / f=O(10)$, the deformation radii associated with the mixed fluid intrusions are small, $L_{R}=O(100) \mathrm{m}$. The laboratory results predict that the coherent advance of mixed fluid into the interior will be arrested at length scales approximately equal to $10 L_{R}$ (4.5). In the examples in Table 6.1 the individual mixed layer exported from the turbulent zone is expected to develop baroclinic instabilities within $1 \mathrm{~km} \pm 200 \mathrm{~m}$ of the boundary. Beyond this penetration distance, further mixing and advection of mixed fluid is independent of interactions at the interface of turbulent and ambient fluid. If these numbers apply, then the circulation initiated by the entrainment and detrainment dynamics is a near field phenomenon. Alternatively, away from the immediate dynamics of the turbulent zone, the stacked layers of mixed fluid might act as a single unit with respect to the ambient stratification and rotation. Then the penetration into the interior would be proportional to the topographic scale, $\mathrm{H}_{\text {region }}$. Although this behavior is not investigated in the current set of experiments, the possibility could be easily tested in a laboratory. 
Table 6.2 presents calculations of mixed fluid volume flux (6.8) for an individual patch of unconfined boundary mixing located at each site. It also lists the flux per unit height within the mixed layer. The last column gives an estimate of volume flux for a vertically integrated stack of mixed layers. The estimate assumes that the vertical dissipation profile is uniform and that the layers are independent of each other.

\begin{tabular}{|l|c|c|c|c|c|}
\hline Site & $\mathrm{Q}\left(\mathrm{m}^{3 / \mathrm{s}}\right)$ & $\mathrm{h}_{\mathrm{m}}$ & $\mathrm{Q} / \mathrm{h}_{\mathrm{m}}$ & $\mathrm{H}_{\text {region }}$ & $\left(\mathrm{Q} / \mathrm{h}_{\mathrm{m}}\right) * \mathrm{H}\left(\mathrm{m}^{3 / \mathrm{s}}\right)$ \\
\hline $\begin{array}{l}\text { MAR } \\
\text { crest }\end{array}$ & 0.07 & 5 & 0.014 & 500 & 7 \\
\hline $\begin{array}{l}\text { MAR } \\
\text { canyon }\end{array}$ & 0.30 & 9 & 0.033 & 500 & 17 \\
\hline $\begin{array}{l}\text { Seamount } \\
\text { flank }\end{array}$ & 0.03 & 2.5 & 0.012 & 200 & 2.4 \\
\hline $\begin{array}{l}\text { Continental slope } \\
\text { canyon }\end{array}$ & 0.50 & 10 & 0.050 & 150 & 7.5 \\
\hline
\end{tabular}

Table 6.2: Calculation of volume flux of mixed fluid for one layer from equation 6.8 , flux per unit layer height, flux over height of dissipation region.

The volume flux per unit length is not easily determined. The laboratory experiments show that the production of mixed fluid is independent of the mixing region length $L_{B}$ for $0.1 \leq L_{f} / L_{B} \leq 0.4$. In the ocean examples this range of $L_{f} / L_{B}$ covers patches between $50 \mathrm{~m}$ and $350 \mathrm{~m}$ long. For mixing patch lengths $<50 \mathrm{~m}$ and $L_{f} / L_{B} \geq 0.4$, the volume flux of mixed fluid will be comparable to the flux observed in the absence of rotation (Equation 4.22). Chapter 4.5 discusses the possibility that mixing dynamics may change for larger values of $L_{f} / L_{B}$. For $L_{f} / L_{B} \ll 0.1$, the laboratory results suggest that lateral entrainment may be unchanged, but vertical entrainment, which is a function of length, may be increasingly significant. In general, the total production of mixed fluid is expected to depend on the number of discrete patches of mixing rather than on the horizontal extent of rough topography. 
The distance between the patches may also affect mixing productivity. The laterally confined experiments show that mixed fluid recirculating within the channel blocks lateral entrainment and curtails mixed fluid production. Even with separation intervals comparable to the length scales of the flow field, $L_{R}$ and $L_{f}$, the distribution of exported fluid from adjacent mixing sites may restrict access of unmixed fluid to individual patches.

The laboratory experiments have only examined the effectiveness of a single patch of mixing, either exposed to the ambient water on three sides or laterally confined between channel walls. The two configurations give the extreme examples of mixed fluid exported directly into the interior or mixed fluid trapped in a recirculating cell. Together they suggest that turbulent patches along ocean boundaries will be characterized by pronounced lateral circulations, layering and possibly complicated interactions. To assess the contribution of localized boundary mixing to overall diapycnal fluxes and large scale ocean circulation requires a greater understanding of the interaction of localized mixing events with each other and with the scales of the topography. Information on the actual distribution of mixing and its effects may be found in observations of intrusions and local recirculations, water mass properties associated with boundaries, and steps in vertical density profiles. Laboratory research can aid in interpreting the observations and the processes behind them. Future work might investigate the collective far field behavior of vertically stacked intrusions, horizontally extended mixing regions and the spacing between regions, and the effects of sloping boundaries on the scales of mixed layers. 


\section{References}

Barcilon, V. and J. Pedlosky, 1967: On the steady motions produced by a stable stratification in a rapidly rotating fluid. J. Fluid Mech., 29, 673-690.

Browand, F. K. and E. J. Hopfinger, 1985: The inhibition of vertical turbulent scale by stable stratification. In: Turbulence and Diffusion in Stable Environments. Ed. J. C. R. Hunt, Oxford University Press, 15-27.

Cacchione, D. and C. Wunsch, 1974: Experimental study of internal waves over a slope. J. Fluid Mech., 66, 223-239.

Davies, P. A., H. J. S. Fernando, P. Besley and R. J. Simpson, 1991: Generation and spreading of a turbulent mixed layer in a rotating, stratified fluid. J. Geophys. Res., 96, 12567-12585.

De Silva, I. P. D., J. Imberger and G. N. Ivey, 1997: Localized mixing due to a breaking internal wave ray at a sloping bed. J. Fluid Mech., 350, 1-27.

De Silva, I. P. D. and H. J. S. Fernando, 1998: Experiments on collapsing turbulent regions in stratified fluids. J. Fluid Mech., 358, 29-60.

Dickey, T. D. and G. L. Mellor, 1980: Decaying turbulence in neutral and stratified fluids. J. Fluid Mech., 99, 13-31.

Dickinson, S. C. and R. R. Long, 1983: Oscillating-grid turbulence including effects of rotation. J. Fluid Mech., 126, 315-333.

Dillon, T. M., 1982: Vertical Overturns: A comparison of Thorpe and Ozmidov length scales. J. Geophys. Res., 87, 9601-9613.

E, X. and E. J. Hopfinger, 1986: On mixing across an interface in stably stratified fluid. $J$. Fluid Mech., 166, 227-244. 
Fernando, H. J. S., 1988: The growth of a turbulent patch in a stratified fluid. J. Fluid Mech., 190, 55-70.

Fleury, M., M. Mory, E. J. Hopfinger and D. Auchere, 1991: Effects of rotation on turbulent mixing across a density interface. J. Fluid Mech., 223, 165-191.

Gregg, M. C., 1980: Microstructure patches in the thermocline. J. Phys. Oceanogr., 10, 915-943.

Gregg, M. C., 1987: Diapycnal mixing in the thermocline: a review. J. Geophys. Res. 92 , 5249-5286.

Gregg, M. C. and T. Sanford, 1980: Signatures of mixing from the Bermuda Slope, the Sargasso Sea, and the Gulf Stream. J. Phys. Oceanogr., 10, 105-127.

Hogg, N., P. Biscaye, W. Gardner and W. J. Schmitz, Jr., 1982: On the transport and modification of Antarctic Bottom Water in the Vema Channel. J. Mar. Res, 40 (Suppl), 231-263.

Hogg, N. G. and W. B. Owens, 1999: Direct measurement of the deep circulation within the Brazil Basin. Deep-Sea Res II, 46, 335-353.

Hopfinger, E. J. and J.-A. Toly, 1976: Spatially decaying turbulence and its relation to mixing across density interfaces. J. Fluid Mech., 78, 155-175.

Hopfinger, E. J., F. K. Browand and Y. Gagne, 1982: Turbulence and waves in a rotating tank. J. Fluid Mech., 125, 505-534.

Ivey, G. N., 1987: Boundary mixing in a rotating, stratified fluid. J. Fluid Mech., 183, 2544.

Ivey, G. N. and G. M. Corcos, 1982: Boundary mixing in a stratified fluid. J. Fluid Mech., 121, 1-26.

Ivey, G. N. and R. L. Nokes, 1989: Vertical mixing due to breaking of critical internal waves on sloping boundaries. J. Fluid Mech., 204, 479-500.

Jacquin, L., O. Leuchter, C. Cambon and J. Mathieu, 1990: Homogeneous turbulence in the presence of rotation. J. Fluid Mech., 220, 1-52.

Kunze, E. and J. M. Toole, 1997: Tidally driven vorticity, diurnal shear, and turbulence atop Fieberling Seamount. J. Phys. Oceanogr., 27, 2663-2693. 
Ledwell, J. R., E. T. Montgomery, K. L. Polzin, L. C. St. Laurent, R. W. Schmitt and J. M. Toole, 2000: Evidence for enhanced mixing over rough topography in the abyssal ocean. Nature, 403, 179-182.

Linden, P. F., 1973: The interaction of a vortex ring in a sharp density interface: a model for turbulent entrainment. J. Fluid Mech., 60, 467-480.

Long, R. R., 1978a: A theory of mixing in a stably stratified fluid. J. Fluid Mech., 84, 113-124.

Long, R. R., 1978b: Theory of turbulence in a homogeneous fluid induced by an oscillating grid. Phys. Fluids, 21, 1887-1888.

Lueck, R. G. and T. D. Mudge, 1997: Topographically induced mixing around a shallow seamount. Science, 276, 1831-1833.

Maxworthy, T., 1986: On turbulent mixing across a density interface in the presence of rotation. J. Phys. Oceanogr., 16, 1136-1137.

Maxworthy, T. and S. G. Monismith, 1988: Differential mixing in a stratified fluid. $J$. Fluid Mech., 189, 571-598.

Morris, M. Y., M. M. Hall, L. C. St. Laurent and N. G. Hogg, 2001: Abyssal mixing in the Brazil Basin. J. Phys. Oceanogr., 31, 3331-3348.

Munk, W., 1966: Abyssal recipes. Deep-Sea Res., 13, 707-730.

Munk, W. and C. Wunsch, 1998: Abyssal recipes II: energetics of tidal and wind mixing. Deep-Sea Res. Part I, 45,1977-2010.

Oster, G., 1965: Density gradients. Scientific American, 213, 70-76.

Ozmidov, R. V., 1965: On the turbulent exchange in a stably stratified ocean. Izv., Atmos. Oceanic Phys., Engl. Transl., 1, 493-497.

Park, Y.-G., J. A. Whitehead and A. Gnanadeskian, 1994: Turbulent mixing in stratified fluids: layer formation and energetics. J. Fluid Mech., 279, 279-311.

Perera, M. J. A. M., H. J. S. Fernando and D. L. Boyer, 1995: Mixing induced by oscillatory stratified flow past a right-circular cylinder. J. Fluid Mech., 284, 1-21. 
Polzin, K. L., J. M. Toole, J. R. Ledwell and R. W. Schmitt, 1997: Spatial variability of turbulent mixing in the abyssal ocean. Science, 276, 93-96.

Polzin, K. and J. Toole, 2002: Subinertial finestructure on the continental slope/rise transition. In preparation.

St. Laurent, L. C., J. M. Toole and R. W. Schmitt, 2001: Buoyancy forcing by turbulence above rough topography in the abyssal Brazil Basin. J. Phys. Oceanogr., 31, 34763495 .

Stommel, H., A. B. Arons and A. J. Faller, 1958: Some examples of stationary planetary flow patterns in bounded basins. Tellus, 10, 179-187.

Taylor, J. R., 1993: Turbulence and mixing in a boundary layer generated by shoaling internal waves. Dyn. Atmos. Oceans, 19, 233-258.

Thomas, P. J. and P. F. Linden, 1996: A laboratory simulation of mixing across tidal fronts. J Fluid Mech., 309, 321-344.

Thorpe, S. A., 1977: Turbulence and mixing in a Scottish Loch. Philos. Trans. R. Soc., London Ser. A, 286, 125-181.

Thorpe, S. A., 1982: On the layers produced by rapidly oscillating a vertical grid in a uniformly stratified fluid. J. Fluid Mech., 124, 391-409.

Toole, J. M., K. L. Polzin and R. W. Schmitt, 1994: Estimates of diapycnal mixing in the abyssal ocean. Science, 264, 1120-1123.

Toole, J. M., R. W. Schmitt and K. L. Polzin, 1997: Near-boundary mixing above the flanks of a midlatitude seamount. J. Geophys. Res. 102, 947-959.

Turner, J. S., 1973: Mixing across density interfaces. In: Buoyancy Effects in Fluids. Cambridge University Press, 288-312.

Turner, J. S., 1986: Turbulent entrainment: the development of the entrainment assumption, and its application to geophysical flows. J. Fluid Mech., 173, 431

Whitehead, J. A. and L. V. Worthington, 1982: The flux and mixing rates of Antarctic Bottom Water within the North Atlantic. J. Geophys. Res. 87, 7903-7924. 